\title{
SYNTHESIS AND CHARACTERIZATION OF BORON INCORPORATED DIAMOND-LIKE CARBON THIN FILMS
}

\author{
A Thesis Submitted to the College of \\ Graduate Studies and Research \\ In Partial Fulfillment of the Requirements \\ For the Degree of Master of Science \\ In the Department of Mechanical Engineering \\ University of Saskatchewan \\ Saskatoon
}

By

LINLIN ZHANG

(C) Copyright Linlin Zhang, August, 2012. All rights reserved. 


\section{PERMISSION TO USE}

In presenting this thesis in partial fulfilment of the requirements for a Postgraduate degree from the University of Saskatchewan, I agree that the Libraries of this University may make it freely available for inspection. I further agree that permission for copying of this thesis in any manner, in whole or in part, for scholarly purposes may be granted by the professor Qiaoqin Yang who supervised my thesis work or, in her absence, by the Head of the Department or the Dean of the College in which my thesis work was done. It is understood that any copying or publication or use of this thesis or parts thereof for financial gain shall not be allowed without my written permission. It is also understood that due recognition shall be given to me and to the University of Saskatchewan in any scholarly use which may be made of any material in my thesis.

Requests for permission to copy or to make other use of material in this thesis in whole or part should be addressed to:

Head of the Department of Mechanical Engineering

University of Saskatchewan

Saskatoon, Saskatchewan S7N 5A9

Canada 


\begin{abstract}
Diamond-like carbon (DLC) thin films have been attracting significant interest from both academic and industrial communities due to their unique structures and properties. Boron (B) incorporation is very promising to modify DLC properties for electronic and mechanical applications. However, the current techniques suffer from their limitations and it's difficult to use them to prepare high quality B incorporated DLC (B-DLC) films to meet application demands. A recently developed biased target ion beam deposition (BTIBD) technique has been applied to produce high quality DLC based films, but no work has been reported on synthesis of B-DLC films by BTIBD and their characteristics.

In this work, B-DLC films were synthesized on silicon wafers using BTIBD technique, where DLC was deposited by ion beam deposition and B was simultaneously incorporated by ion beam sputtering of boron carbide $\left(\mathrm{B}_{4} \mathrm{C}\right)$ under different conditions. DLC films and B-carbon (B-C) films were also synthesized by ion beam deposition and ion beam sputtering of $\mathrm{B}_{4} \mathrm{C}$ under similar conditions, respectively, as reference samples and for estimation of $\mathrm{B}_{4} \mathrm{C}$ sputtering rate and DLC deposition rate to control the B content in B-DLC. Scanning electron microscopy, atomic force microscopy, X-ray diffraction, Raman spectroscopy, synchrotron-based X-ray photoelectron spectroscopy and near edge X-ray absorption fine structure were applied to investigate morphology, microstructure, chemical composition and bonding state of the films. Nanoindentation and ball-on-disc tests were conducted using a Universal Mechanical Tester to measure hardness, Young's modulus and friction coefficient of the synthesized films. The preliminary relationships between processing conditions, film structures and properties were investigated.
\end{abstract}


B-DLC thin films with different B doping levels (up to 8 wt. \%) have been successfully synthesized by the BTIBD technique and the B concentration incorporated increases with the increase of target bias voltage and sputtering ion current density. It has been found that B exists in different states in B-DLC, including carbon-rich and B-rich boron carbides, boron suboxide and boron oxide, and the oxidation of B probably occurs during the film deposition. The incorporation of B into DLC leads to the increase of $\mathrm{sp}^{3}$ bonded carbon in the films, the increase of both film hardness and elastic modulus, and the decrease of both surface roughness and friction coefficient. Furthermore, the content of $\mathrm{sp}^{3}$ bonded carbon, film hardness and elastic modulus increase, and the film surface roughness and friction coefficient decrease with the increase of B-rich B-C bonding content in the B-DLC films. The results demonstrate a good promise to synthesize quality B-DLC films using BTIBD. 


\section{ACKNOWLEDGMENTS}

I would love to acknowledge my supervisor Prof. Qiaoqin Yang for her invaluable guidance and encouragement throughout my graduate study, as well as for her keen care about my personal development and career. I want to express my appreciation to my Advisory Committee members, Prof. Jerzy A. Szpunar and Prof. Akindele G. Odeshi, for their great guidance and suggestions.

My special thanks go to Dr. Yongji Tang and Dr. Yuanshi Li for their help and instructions in my experiments and thesis work. My thanks also go to the members of our research group for their help and support on my work. Meantime I would like to thank those who provided me with important technical assistance in using the film synthesis and characterization facilities, including Mr. Robert Peace from the Department of Mechanical Engineering, Dr. Jason Maley from the Saskatchewan Structural Sciences Centre, Dr. Yongfeng Hu and Dr. Xiaoyu Cui from the Canadian Light Source Inc.

Financial assistance provided by the Canada Research Chair Program, the Natural Sciences and Engineering Research Council of Canada and the University of Saskatchewan is gratefully acknowledged.

Finally, my sincere acknowledgement and appreciation go out to my husband, daughter, parents, brother and in-laws for their constant love and support throughout my graduate study. 


\section{DEDICATION}

This work is dedicated to my husband Jiwei Li, daughter Muen Li

$\&$

beloved parents Fengqin Shi and Qunchao Zhang 


\section{TABLE OF CONTENTS}

page

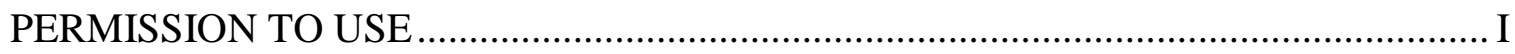

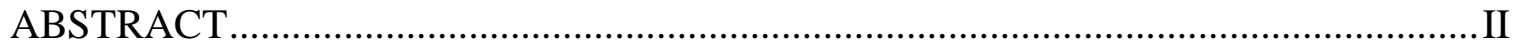

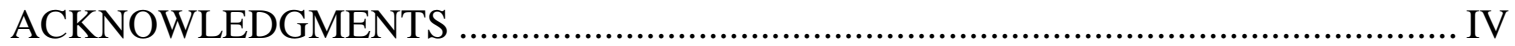

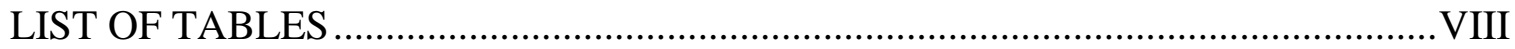

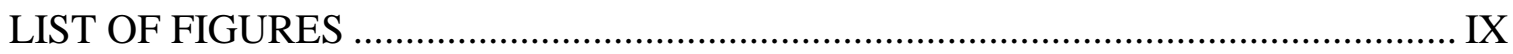

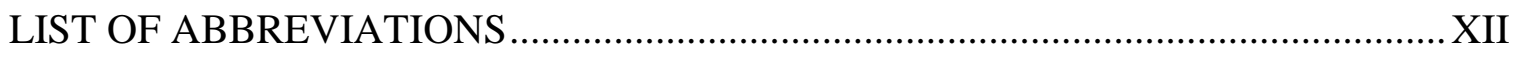

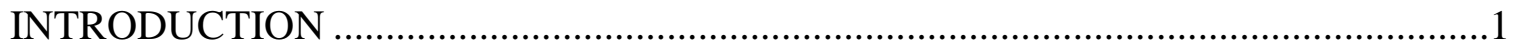

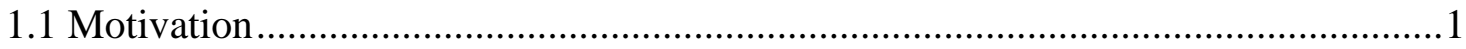

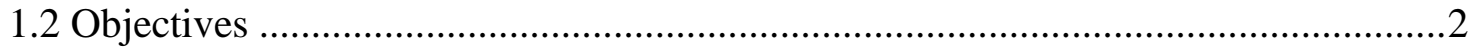

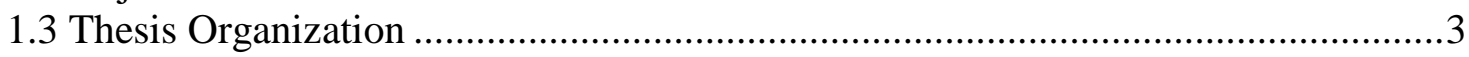

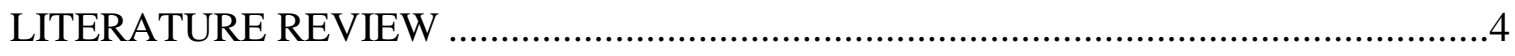

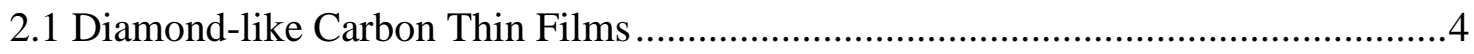

2.1.1 Carbon Materials ..................................................................................... 4

2.1.2 Types, Structure Models and Properties of DLC Thin Films .......................... 6

2.1.3 DLC Deposition Mechanism .................................................................. 9

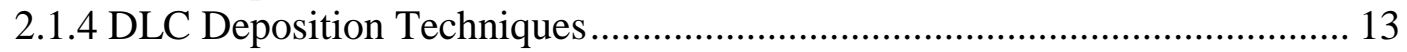

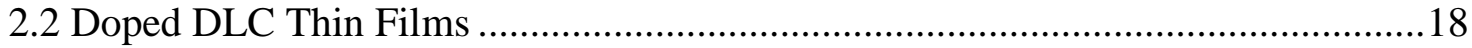

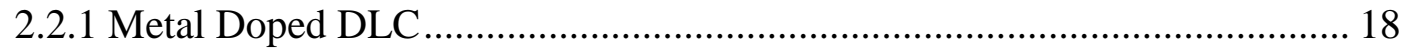

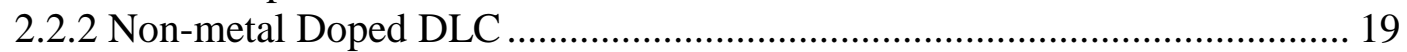

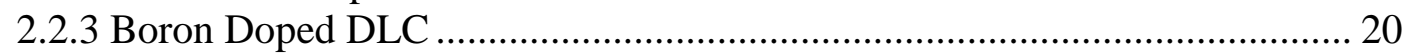

2.3 Biased Target Ion Beam Deposition of DLC Based Thin Films ............................22

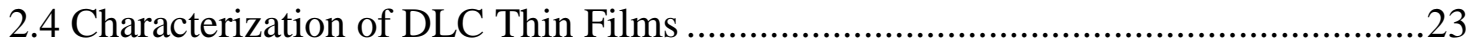

2.4.1 Scanning Electron Microscopy …………………................................. 23

2.4.2 Atomic Force Microscopy....................................................................... 26

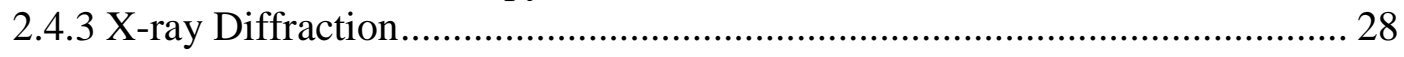

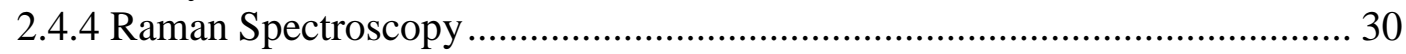

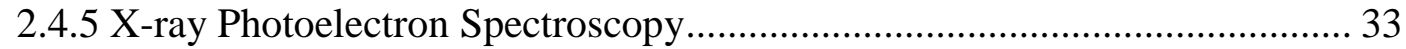

2.4.6 Near Edge X-ray Absorption Fine Structure.................................................. 34

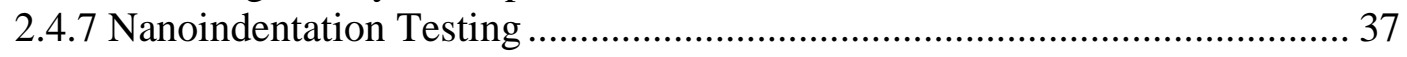

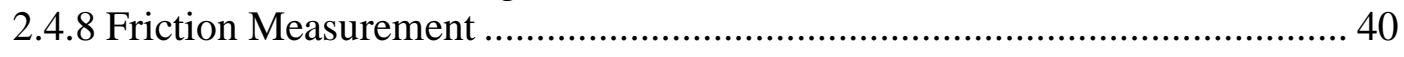

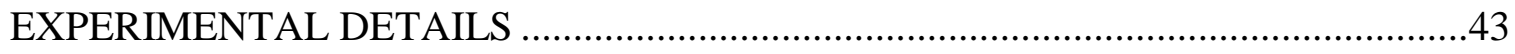

3.1 Biased Target Ion Beam Deposition System .........................................................43

3.2 B-C Thin Film Deposition by Ion Beam Sputtering of $\mathrm{B}_{4} \mathrm{C}$ Target ………….........46

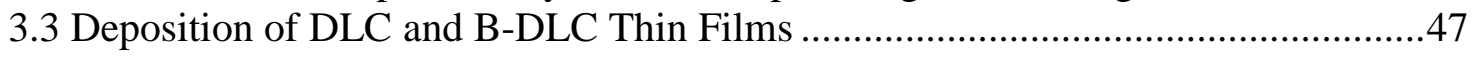

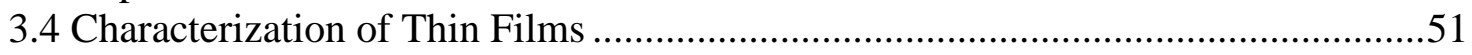

3.4.1 Chemical and Structural Characterization .................................................. 51

3.4.2 Mechanical and Friction Characterization ……………….......................... 55

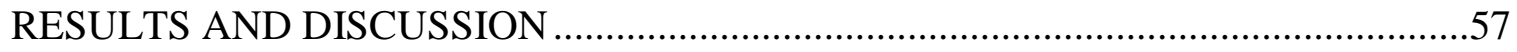




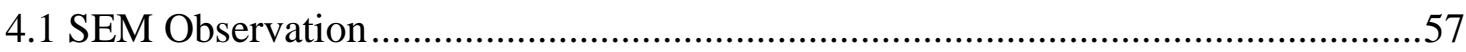

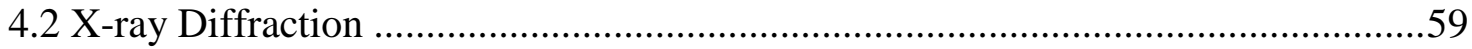

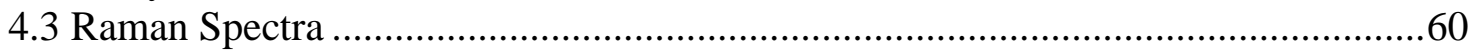

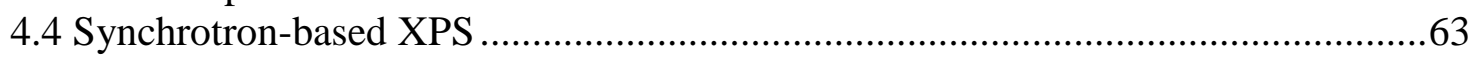

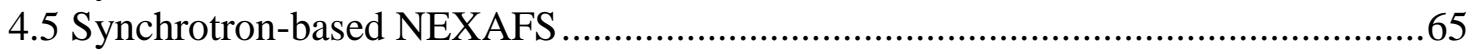

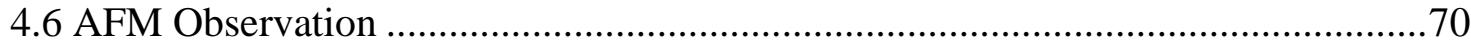

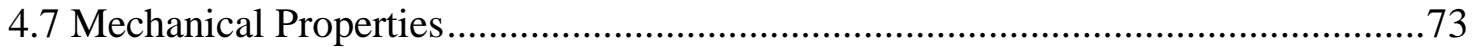

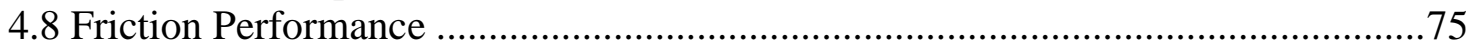

SUMMARY, CONCLUSIONS AND FUTURE WORK ............................................78

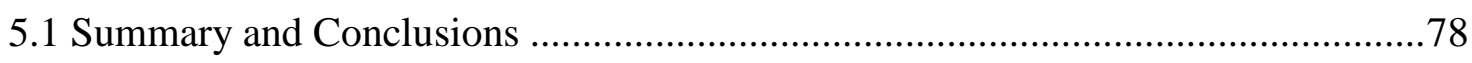

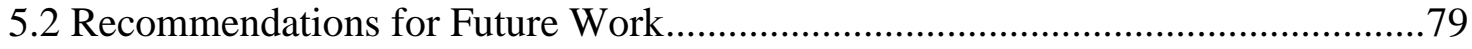

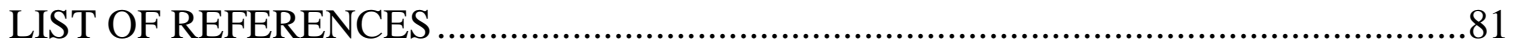




\section{LIST OF TABLES}

$\underline{\text { Table }}$

page

Table 3.1 Sputtering parameters and estimated deposition rates on silicon ....................47

Table 3.2 DLC deposition parameters on silicon ....................................................48

Table 3.3 Deposition parameters for B-DLC films on silicon ...................................50

Table $4.1 \mathrm{I}_{\mathrm{d}} / \mathrm{I}_{\mathrm{g}}$ ratios for DLC and B-DLC thin films .........................................62

Table 4.2 Measured surface roughness for DLC and B-DLC films ..............................70 


\section{LIST OF FIGURES}

$\underline{\text { Figure }}$

page

Figure 2.1 Bonding configurations of carbon atoms

(http://www.chemistryland.com/CHM151S/09-CovalentBonds/Covalent.html,

2012-1-5)

Figure 2.2 Carbon allotropes (http://www.realmagick.com/carbon-allotropes, 2012-1-6) .5

Figure 2.3 Ternary diagram of the $\mathrm{C}, \mathrm{H}$ system showing various forms of DLC

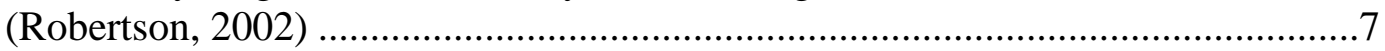

Figure 2.4 Schematic of $\mathrm{sp}^{2}$ clusters in a-C:H film (Robertson, 2002) ...........................

Figure 2.5 Molecular dynamic simulation of a-C:H film atomic structure (Erdemir et al.,

2006)

Figure 2.6 Component processes in a-C:H film growth (Robertson, 2002) ....................12

Figure 2.7 Schematics of various DLC deposition methods (Robertson, 2002) ..............14

Figure 2.8 (a) Ion beam assisted electron beam evaporation

(http://cim.aamu.edu/Activities/ibad.html, 2012-1-20); (b) Dual ion beam

deposition (http://web.mit.edu/cprl/www/dualion1.gif, 2012-1-20)

Figure 2.9 Schematic of SEM

(http://www.chm.bris.ac.uk/pt/diamond/stuthesis/chapter2.htm, 2012-2-6) ........25

Figure 2.10 Schematic of AFM

(http://en.wikipedia.org/wiki/File:Atomic_force_microscope_block_diagram.svg,

2012-2-8)

Figure 2.11 Schematic of XRD (http://tap.iop.org/atoms/xray/530/page_47297.html,

2012-2-10).

Figure 2.12 Schematic of a Raman spectrometer (http://www.chm.bris.ac.uk/pt/diamond/stuthesis/chapter2.htm, 2012-2-13) .......31

Figure 2.13 Typical Raman spectra of various carbon materials (Robertson, 2002) ........32

Figure 2.14 Schematic of electron transitions by X-ray incident on a material

(https://commons.wikimedia.org/wiki/File:Photoabsorb.png, 2012-2-16)

Figure 2.15 Universal Nano+Micro Tester

(http://www.cetr.com/Brochures/Nano-Indentation/determination_of_storage_

and_los_moduli_UNMT.htm, 2012-2-20). 
Figure 2.16 Schematic of pin-on-disc sliding testing (http://tribolab.mas.bg.ac.rs/english/images/equipment/T-62_sketch.jpg, 2012-2-22).

Figure 3.1 Picture of the BTIBD system

Figure 3.2 Schematic of the BTIBD system .............................................................44

Figure 3.3 Scanning electron microscopy - JEOL JSM-6010LV .................................52

Figure 3.4 Atomic force microscopy - PicoSPM ..................................................52

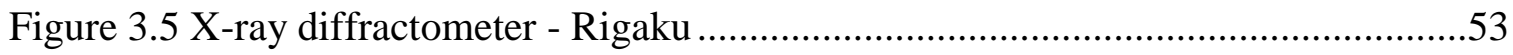

Figure 3.6 Raman spectroscope - Renishaw model 2000 .........................................54

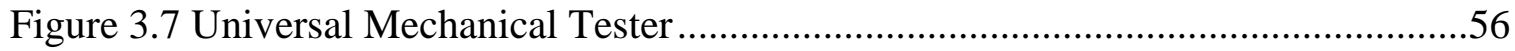

Figure 4.1 Cross-section SEM image of B-C-1 (calculated film thickness $0.61 \mu \mathrm{m}$ ) .......57

Figure 4.2 Cross-section SEM image of B-C-3 (calculated film thickness $0.12 \mu \mathrm{m}$ ) .......58

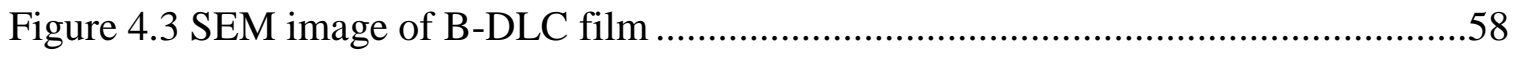

Figure 4.4 Typical XRD pattern of B-C thin film samples by $\mathrm{B}_{4} \mathrm{C}$ sputtering .................59

Figure 4.5 Raman spectra of pure DLC and B-DLC films .........................................60

Figure 4.6 Gaussian-fitted Raman spectrum of DLC film $\left(\mathrm{I}_{\mathrm{d}} / \mathrm{I}_{\mathrm{g}}=1.01\right) \ldots \ldots \ldots \ldots \ldots \ldots \ldots \ldots \ldots \ldots \ldots \ldots \ldots \ldots \ldots . . .61$

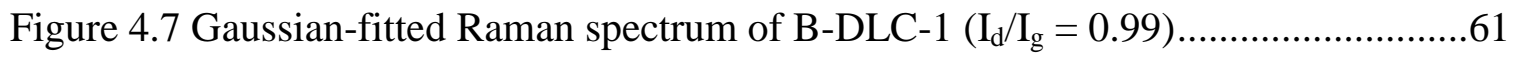

Figure 4.8 Gaussian-fitted Raman spectrum of B-DLC-5 $\left(\mathrm{I}_{\mathrm{d}} / \mathrm{I}_{\mathrm{g}}=0.92\right) \ldots \ldots \ldots \ldots \ldots \ldots \ldots \ldots \ldots \ldots \ldots \ldots \ldots \ldots \ldots \ldots . . .62$

Figure 4.9 XPS spectra of $\mathrm{B}_{4} \mathrm{C}$ sputtered film and B-DLC films ...............................64

Figure 4.10 XPS spectra of C 1s for B-DLC films ..................................................65

Figure 4.11 B K-edge NEXAFS in FLY mode for B-DLC films and in TEY mode for

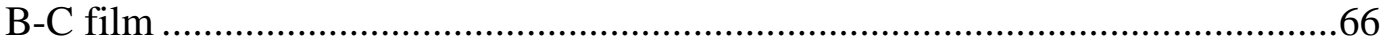

Figure 4.12 C K-edge NEXAFS in TEY mode for B-C film and B-DLC films ..............69

Figure 4.13 C K-edge NEXAFS in FLY mode for B-DLC films ................................69

Figure 4.14 AFM image of DLC film (Si wafer 3) ..............................................

Figure 4.15 AFM images of B-DLC-1 …....................................................... 


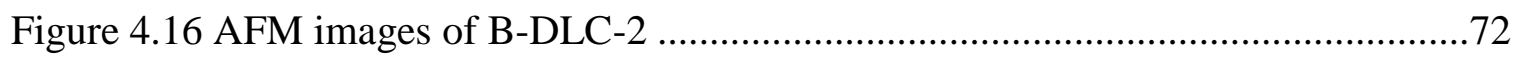

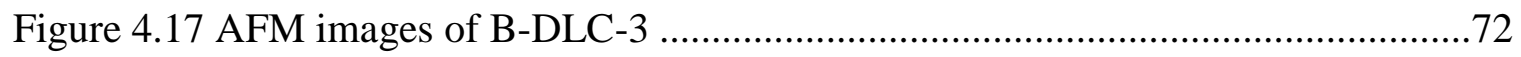

Figure 4.18 (a) Hardness and (b) Young's modulus of DLC and B-DLC films ..............74

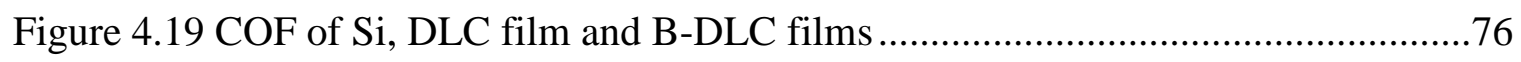




\section{LIST OF ABBREVIATIONS}

a-C Hydrogen free amorphous carbon

a-C:H Hydrogenated amorphous carbon

AFM Atomic force microscopy

Ar

Argon

B

Boron

$\mathrm{B}_{4} \mathrm{C}$

Boron carbide

$\mathrm{B}-\mathrm{C}$

B-carbon

B-DLC B incorporated diamond-like carbon

BSE Back-scattered electrons

BTIBD Biased target ion beam deposition

C

Carbon

$\mathrm{CH}_{4} \quad$ Methane

CLS Canadian Light Source Inc.

$\mathrm{CN}_{\mathrm{x}} \quad$ Carbon nitride

Co Cobalt

COF Coefficient of friction

$\mathrm{Cr} \quad$ Chromium

CVD Chemical vapor deposition

DB Dangling bond

DLC Diamond-like carbon

EH End-Hall

F Fluorine

FLY Fluorescence yield 


$\begin{array}{ll}\text { H } & \text { Hydrogen } \\ \text { Mo } & \text { Molybdenum } \\ \text { MSIBD } & \text { Mass-selected ion beam deposition } \\ \text { N } & \text { Nitrogen } \\ \text { NEXAFS } & \text { Near edge X-ray absorption fine structure } \\ \text { Ni } & \text { Nickel } \\ \text { O } & \text { Oxygen } \\ \text { PVD } & \text { Physical vapor deposition } \\ \text { RMS } & \text { Root mean square } \\ \text { SEM } & \text { Scanning electron microscopy } \\ \text { SGM } & \text { Spherical grating monochromator } \\ \text { Si } & \text { Silicon } \\ \text { SSSC } & \text { Saskatchewan Structural Science Centre } \\ \text { ta-C } & \text { Tetrahedral hydrogen free amorphous carbon } \\ \text { ta-C:H } & \text { Tetrahedral hydrogenated amorphous carbon } \\ \text { TEY } & \text { Total electron yield } \\ \text { Ti } & \text { Titanium } \\ \text { UMT } & \text { Universal Mechanical Tester } \\ \text { X } & \text { Xungsten } \\ \text { XAS } & \text { X-ray absorption spectroscopy } \\ \text { XPSotoelectron spectroscopy } & \\ & \end{array}$




\section{CHAPTER 1 \\ INTRODUCTION}

\subsection{Motivation}

Diamond-like carbon (DLC) thin films have been attracting significant interest from both academic and industrial communities due to their unique properties, such as high hardness, high wear and corrosion resistance, low friction coefficient, high chemical inertness, optical transparency and good biocompatibility (Nakazawa et al., 2010). Especially, due to their amorphous nature, their properties, including thermal stability, hardness, internal stress, tribological property, electrical conductivity, surface energy, biocompatibility, adhesion, can be finely tailored to desired values for specific applications by the incorporation of different elements in their structures (Sikora et al., 2009a). Those elements include metals, e.g. titanium (Ti), tungsten (W), cobalt (Co), nickel (Ni) (VijaiBharathy et al., 2010a, 2010b \& 2011; Tang et al., 2011a), nonmetals, e.g. boron (B), silicon ( $\mathrm{Si})$, nitrogen $(\mathrm{N})$, fluorine $(\mathrm{F})$, and their combinations (Erdemir et al., 2006).

$\mathrm{B}$ incorporation to DLC has been receiving increasing research interest because B incorporated DLC (B-DLC) thin films have been found to exhibit increased thermal stability (Monteiro, 2001), improved electron field emission (Cheng et al., 1999), increased hardness and reduced film stress (He et al., 2000) with great potential for electronic and mechanical applications. The techniques currently used to synthesize B-DLC films include magnetron sputtering, mass-separated ion beam deposition, plasma-enhanced chemical vapor deposition (CVD), and pulsed laser deposition. However, those techniques suffer from their limitations and it's difficult to use them to prepare high quality B-DLC films to meet the demands of many applications. For example, it is difficult to produce ultra-smooth DLC as protective coatings for magnetic storage application or low contamination DLC for high speed microelectronics by the 
widely used magnetron sputtering method. Therefore, there is a need to develop new processes that could synthesize B-DLC films with higher quality. In addition, the structure of B-DLC and its effects on the film properties have not been fully investigated and understood.

Recently, a biased target ion beam deposition (BTIBD) technique has been developed to overcome some drawbacks of conventional sputtering methods. It can operate at lower pressure (one order of magnitude lower than that in magnetron sputtering) and low temperature (without any extra heating, close to room temperature), and has much less film contaminations due to the use of gridless end-Hall (EH) ion source instead of the gridded ion sources in regular ion beam deposition systems and the use of biased target ion beam sputtering instead of magnetron sputtering (Tang et al., 2011a). This technique has been used to produce smooth, uniform, and high quality DLC based films including pure and N incorporated DLC (Sethuraman, 2009), Co incorporated DLC (Tang et al., 2011a), Ti incorporated DLC (VijaiBharathy et al., 2010a), W incorporated DLC (VijaiBharathy et al., 2011), and Ni incorporated DLC (VijaiBharathy et al., 2010b). However, no work has been reported on the synthesis of B-DLC films by BTIBD and their characteristics.

\subsection{Objectives}

The overall goal of this thesis work is to synthesize B-DLC thin films with well controlled B concentration using the newly developed BTIBD technique, and to understand the effects of B incorporation on the structure and properties of the synthesized films. The specific research includes:

1. Synthesis of pure hydrogenated DLC thin films using EH ion beam deposition;

2. Synthesis of B-carbon (B-C) thin films by BTIBD using biased target ion beam sputtering; 
3. Synthesis of B-DLC thin films with different B contents by BTIBD using ion beam deposition of DLC plus simultaneous biased target sputtering of boron carbide $\left(\mathrm{B}_{4} \mathrm{C}\right)$;

4. Evaluation of the composition, structure, and properties of the synthesized thin films using scanning electron microscopy (SEM), atomic force microscopy (AFM), X-ray diffraction (XRD), Raman spectroscopy, synchrotron-based X-ray photoelectron spectroscopy (XPS) and near edge X-ray absorption fine structure (NEXAFS), nanoindentation and ball-on-disk testing;

5. Understanding the effects of B incorporation to DLC on the structure and properties of the films.

\subsection{Thesis Organization}

This thesis consists of five chapters. Chapter 1 briefly introduces the motivation for my thesis work, presents the research objectives, and outlines the thesis organization. Chapter 2 provides a detailed literature review of the research area, including the structure, properties, synthesis, applications, and main characterization techniques of DLC and doped DLC thin films. Chapter 3 gives the experimental details including equipment, methods and parameters for the film synthesis and characterization. Chapter 4 focuses on the experimental results and discussion of my thesis work, containing surface morphology, microstructure, chemical composition, and bonding state of the synthesized films as well as the film mechanical properties and friction performance. The last Chapter presents the summary and conclusions of the research work which has been done and makes recommendations for future work. 


\section{CHAPTER 2 \\ LITERATURE REVIEW}

\subsection{Diamond-like Carbon Thin Films}

\subsubsection{Carbon Materials}

Carbon (C) is well-known in its diversity of bonding configurations. There are four electrons in the outermost shell of carbon atoms, which can be shared, in most cases, to form covalent bonds with other atoms including other carbon atoms. Figure 2.1 shows three different bonding configurations of carbon atoms. The first bonding configuration is called $\mathrm{sp}^{3}$ hybridization, which is tetrahedrally directed four-fold configuration of $\sigma$-type bond. The second one is called $\mathrm{sp}^{2}$ hybridization, which is trigonally directed three-fold planar configuration containing both $\sigma$ and $\pi$ type bonds. The third bonding configuration $\mathrm{sp}^{1}$ corresponds to linear geometry consisting of both $\sigma$ and $\pi$ bonds.

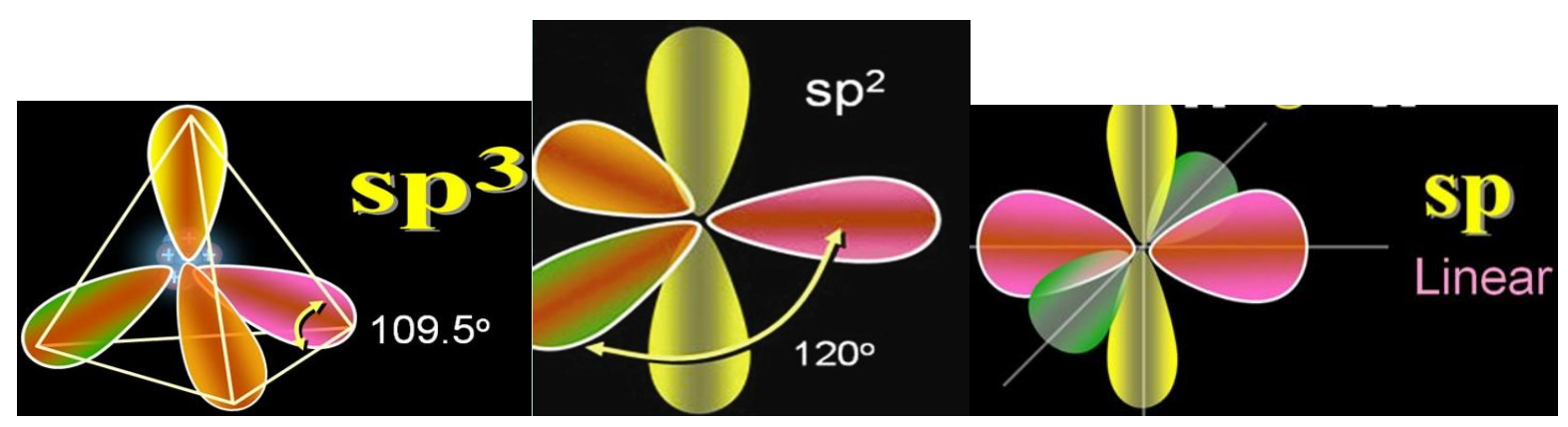

Figure 2.1 Bonding configurations of carbon atoms

(http://www.chemistryland.com/CHM151S/09-CovalentBonds/Covalent.html, 2012-1-5)

Based on the different bonding configurations, carbon forms various allotropes of which the best known are graphite $\left(\mathrm{sp}^{2}\right)$, diamond $\left(\mathrm{sp}^{3}\right)$, nanostructures, and amorphous carbon (mixture of $\mathrm{sp}^{2}$ and $\mathrm{sp}^{3}$ ). Those allotropes have different structures and vary widely in their major properties. Figure 2.2 displays the atomic structure of some carbon allotropes. 


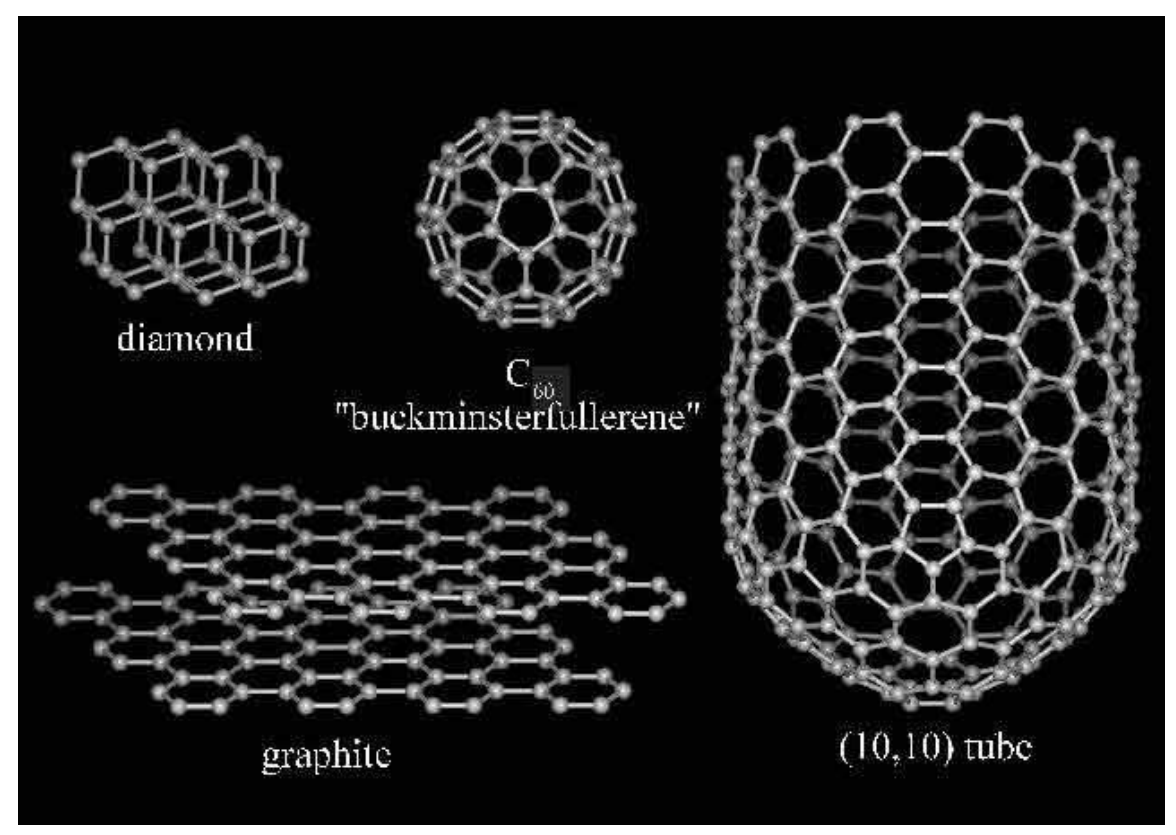

Figure 2.2 Carbon allotropes (http://www.realmagick.com/carbon-allotropes, 2012-1-6)

Diamond has $\mathrm{sp}^{3}$ hybridization and all the four carbon bonds in diamond are symmetrically equivalent and strong. The tetrahedral carbon bonds in diamond crystallize into an inflexible cubic face-centered crystalline lattice. Originating from its special structure and strong bonding, diamond has exceptional characteristics, including extreme hardness, highest thermal conductivity, high transparency, high chemical inertness and very low electrical conductivity. Graphite has $\mathrm{sp}^{2}$ hybridization and the three planar carbon bonds in graphite are strong and equivalent. Graphite has a crystalline structure where planar layers of hexagonal carbon structure are connected to each other by weak van der Waals forces. The planar layers may slide easily over each other causing overall structure weak. Due to its different structure, graphite has different properties compared to diamond. It is opaque and black, electrically conductive, and soft enough to draw a streak on paper. Both diamond and graphite are valuable in industrial applications.

Carbon can also exist as nanostructures, e.g. Buckminsterfullerene (C60) and carbon nanotube, or amorphous structure. C60 is in a shape of soccer ball consisting of sixty carbon atoms 
covalently bonded in hexagonal and pentagonal rings. C60 can form a semiconductor solid by van der Waals forces. It can also form films with strong non-linear optical properties (optical absorption increasing with light intensity) for photovoltaic applications. Carbon nanotube has a cylindrical nanostructure with a single rolled layer or multiple rolled layers of one-atom-thick graphite sheet. It consists of $\mathrm{sp}^{2}$ bonding hybridization and has extremely high strength and very good thermal conductivity. Amorphous carbon consists of a random network of carbon atoms with different bonding hybridizations. They can be graphite-like or diamond-like depending on their bonding states (Robertson, 1986). Graphite-like carbon contains a large amount of $\mathrm{sp}^{2}$ bonded carbon with properties similar to graphite, whereas diamond-like carbon consists of a significant fraction of $\mathrm{sp}^{3}$ bonded carbon with properties similar to diamond.

\subsubsection{Types, Structure Models and Properties of DLC Thin Films}

DLC is normally defined as carbon based thin films with a high fraction of metastable $\mathrm{sp}^{3}$ C-C bonds (Robertson, 2002). A common type of DLC is hydrogenated amorphous carbon (a-C:H) with a moderate $\mathrm{sp}^{3}$ content and a hydrogen $(\mathrm{H})$ content of $10-40 \%$ (Lifshitz, 2003), or highly tetrahedral hydrogenated amorphous carbon (ta-C:H) with a high $\mathrm{sp}^{3}$ content and a relatively low hydrogen content. Another important type of DLC is hydrogen free amorphous carbon (a-C) films consisting of a significant amount of $\mathrm{sp}^{2}$ hybridized bonds or highly tetrahedral hydrogen free amorphous carbon (ta-C) with a predominance of $\mathrm{sp}^{3} \mathrm{C}-\mathrm{C}$ bonding up to $90 \%$ (Lifshitz, 2003). Figure 2.3 is a ternary diagram of C, H, showing the various forms of DLC with different $\mathrm{sp}^{3}$ and $\mathrm{H}$ contents. 


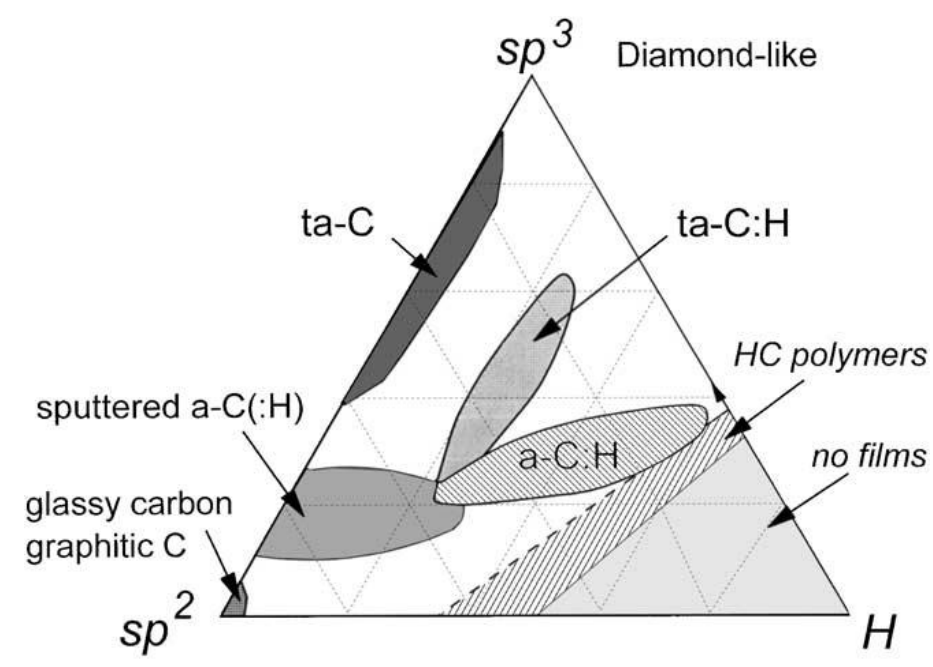

Figure 2.3 Ternary diagram of the $\mathrm{C}, \mathrm{H}$ system showing various forms of DLC (Robertson, 2002)

The bonding in DLC is predominated by $\mathrm{sp}^{2}$ and $\mathrm{sp}^{3}$ (Erdemir et al., 2006). Various models have been developed for understanding the bonding in DLC as well as its electronic structure. Robertson proposed a cluster model giving a pretty simple view of DLC bonding and structure. In the cluster model, the $\mathrm{sp}^{2}$ sites in planar clusters of certain size are embedded in a $\mathrm{sp}^{3}$ bonded network or matrix. The $\mathrm{sp}^{2}$ cluster arrangement determines DLC electronic properties and optical gap, and the $\mathrm{sp}^{3}$ matrix determines DLC mechanical properties (Robertson, 2002). A schematic of the cluster model is shown in Figure 2.4. Although there have been many models proposed, the detailed atomic scale structure of DLC has not been experimentally determined. Figure 2.5 displays a molecular dynamic simulation of the structure of a typical a-C:H film (containing approximate 10 at. $\% \mathrm{H})$, which has a carbon and hydrogen network with three- $\left(\mathrm{sp}^{2}\right)$ and four-fold $\left(\mathrm{sp}^{3}\right)$ atomic coordination (Erdemir et al., 2006). 


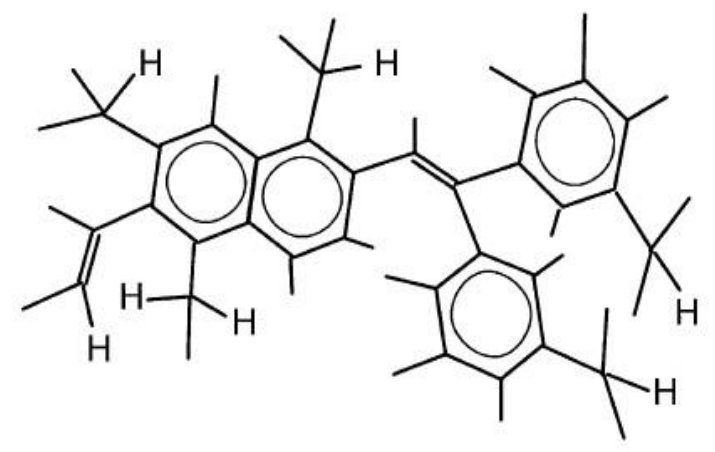

Figure 2.4 Schematic of $\mathrm{sp}^{2}$ clusters in a-C:H film (Robertson, 2002)

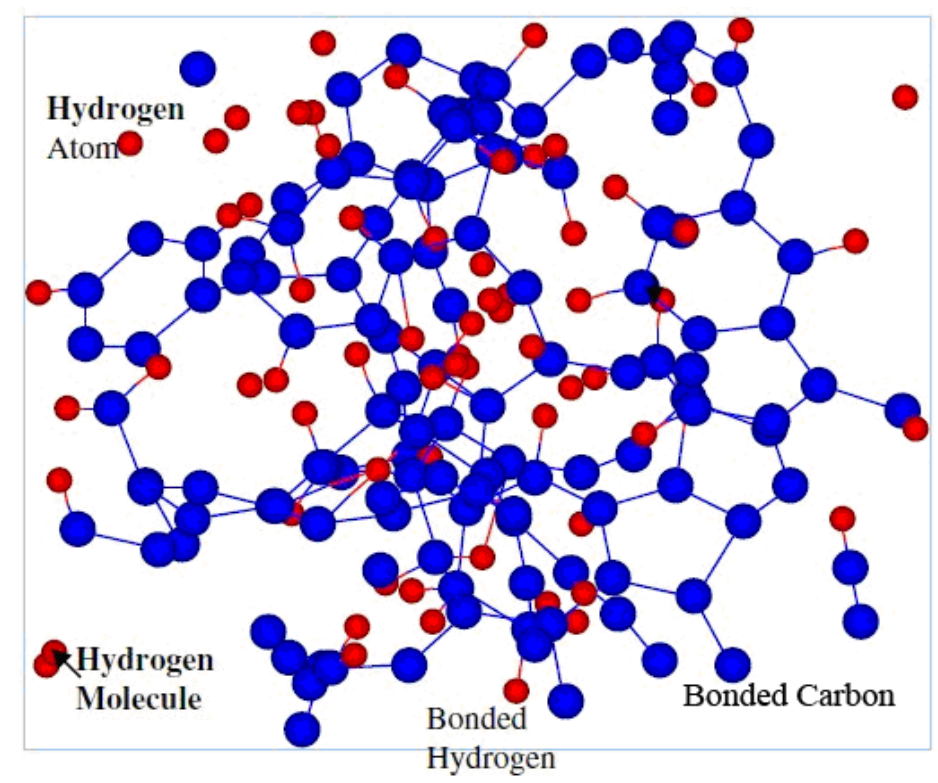

Figure 2.5 Molecular dynamic simulation of a-C:H film atomic structure (Erdemir et al., 2006)

As an amorphous phase, DLC is a random network of atoms with different bonding components, including $\mathrm{sp}^{3} \mathrm{C}-\mathrm{C}, \mathrm{sp}^{2} \mathrm{C}-\mathrm{C}$, and $\mathrm{C}-\mathrm{H}$ bonds. The hardness and Young's modulus and other general "diamond-like" properties arise from the $\mathrm{sp}^{3} \mathrm{C}-\mathrm{C}$ bonds. Usually, the higher the fraction of the $\mathrm{sp}^{3} \mathrm{C}-\mathrm{C}$ bonds, the higher the hardness and Young's modulus of the DLC film (Robertson, 2002). Because of the high fraction of $\mathrm{sp}^{3} \mathrm{C}-\mathrm{C}$ bonding, DLC thin films generally exhibit properties close to diamond, such as high hardness, high Young's modulus, low friction coefficient, high chemical inertness, and excellent optical transparency and biocompatibility (Nakazawa et al., 2010). However, the chemical composition and bonding state of DLC vary 
significantly depending on the deposition method, precursors and processing parameters (Monteiro, 2001). Therefore, the properties of DLC films have large variations. Generally a-C:H films are softer, less internal-stressed and deposited more thickly compared with hydrogen free DLC films (Erdemir et al., 2006). The thermal stability of a-C:H films is inferior to that of hydrogen free films mainly because they contain hydrogen (Nakazawa et al., 2010). a-C:H films may have a large amount of hydrogen in their structure if they are produced from a hydrocarbon source like methane $\left(\mathrm{CH}_{4}\right)$. It should be mentioned that due to the ubiquitous nature of hydrogen, it is very rare to find purely and truly hydrogen free films, so generally speaking, "hydrogen free" means low hydrogen content in the films (usually below 5\%) and no hydrogenated species involved in the deposition process (Monteiro, 2001).

\subsubsection{DLC Deposition Mechanism}

A distinct character of DLC is its high fraction of $\mathrm{sp}^{3} \mathrm{C}-\mathrm{C}$ bonding, which is metastable at the deposition conditions. It has been found that the key to the formation of metastable $\mathrm{sp}^{3} \mathrm{C}-\mathrm{C}$ bonding is the bombardment of energetic particles during the deposition (Robertson, 2002). In the deposition process, the impinging particles enter subsurface atomic sites to form $\mathrm{sp}^{3}$ bonding and induce a quenched-in density increase. The average impact energy of the impinging particles, strictly, the average energy per incident $\mathrm{C}$ ions/atoms determines the amount of $\mathrm{sp}^{3}$ bonding as well as the hydrogen content in DLC films. The average impact energy for DLC deposition generally ranges from $10-1000 \mathrm{eV}$ (Robertson, 1994). It should be noted that for a-C:H it also depends on the precursor hydrocarbon gases (Monteiro, 2001).

It has been proposed that the DLC film deposition from energetic particles is a subplantation (shallow implantation) process, instead of a conventional surface condensation process (Lifshitz, 1999). The whole subplantation process generally involves four stages: bombarding particles 
penetration to subsurface layers, carbon and/or hydrogen atoms incorporation inducing local stresses, sputtering and dilution of target atoms till formation of a DLC layer, and DLC layer growth upon successive bombardment (Lifshitz, 1999). The basic idea of subplantation has been proven by many numerical and analytical simulations. In subplantation models, the impinging particle energy and the interplay between densification by impinging particles and density relaxation determine the film structure. Robertson reported that the optimum energy for DLC deposition with high $\mathrm{sp}^{3}$ bonding is approximately $100 \mathrm{eV}$ at which the carbon atoms have appropriate energy to penetrate substrate surface without annealing out surface density increment (Robertson, 1994). Erdemir et al. also published that regardless of the deposition techniques used, in ion-dominated processes the highest $\mathrm{sp}^{3}$ bonding fractions are obtained with an average ion energy of approximately $100 \mathrm{eV}$ (Erdemir et al., 2006). Excess bombarding energy can result in a gradual suppression on the formation of $\mathrm{sp}^{3}$ bonding.

For deposition of hydrogen free DLC films at room temperature, a threshold ion energy is required to penetrate to subsurface layers and facilitate $\mathrm{sp}^{3}$ bond formation (Lifshitz, 1996). The $\mathrm{C}$ ions at the threshold energy arrive at subsurface sites and stop due to energy loss by elastic collisions with target atoms, ionization and phonon excitations (called nuclear stopping). Then the $\mathrm{C}$ ions diffuse, react and incorporate with the target atoms to increase the local density and reform the local bonding inducing an increase of $\mathrm{sp}^{3}$ hybridization. Local relaxation and detrapping of the $\mathrm{C}$ ions may remove some of the densification and permit local modification in the highly dense region where the $\mathrm{C}$ ions are embedded. When a maximum density is reached, plastic deformation may occur and set the density and $\mathrm{sp}^{3}$ fraction to a constant value upon further ion incorporation. With impinging ion energy decreasing, the $\mathrm{C}$ ions are trapped on substrate surface, which can result in surface processes, graphitization (increase of $\mathrm{sp}^{2}$ bonding) 
and roughing, and the amount of the C ions increases with ion energy decreasing (Lifshitz, 1996). With impinging ion energy increasing (higher than the threshold energy), the $\mathrm{C}$ ions penetrate deeper into the surface suppressing the formation of $\mathrm{sp}^{3}$ bonding, and the final subsurface sites which the $\mathrm{C}$ ions occupy determine the structure and properties of the evolving DLC film (Lifshitz, 1996).

In terms of depositing a-C:H films, the ion action is still via subplantation. The action and process are more complex, including a film growth step, ion-induced dehydrogenation and compression of C-C skeleton (Robertson, 1994). Figure 2.6 shows the component processes in a-C:H film growth (DBs in the figure means dangling bonds). Different hydrocarbon gases such as methane, ethane, acetylene, benzene, ethylene and propane can be used to deposit a-C:H films. The energetic molecular ions are ionized from the source gases and form an ion beam to be directed onto the substrate surface. They break up into atomic ions with energy distributed evenly and then the formed atomic ions with that energy will subplant respectively. Normally, not only the atomic ions, but also neutrals (such as undissociated precursor gases, mono-radicals like $\mathrm{CH}_{3}$, di-radicals and other unsaturated species like $\mathrm{C}_{2} \mathrm{H}_{4}$ or $\mathrm{C}_{2} \mathrm{H}_{2}$ ) and atomic hydrogen incident on the substrate surface. The neutral species contribute to film growth through directly inserting into surface $\mathrm{C}-\mathrm{C}$ or $\mathrm{C}-\mathrm{H}$ bonds, or reacting with the film upon DBs on the surface to form C-C bonds. Removing $\mathrm{H}$ from a surface $\mathrm{C}-\mathrm{H}$ bond by ion displacing or $\mathrm{H}$ abstraction can create a DB. Not like the neutral hydrocarbon species working only at the surface, both atomic hydrogen and ions penetrate the film. Very small $\mathrm{H}$ atoms penetrate a few nanometers into the film and abstract $\mathrm{H}$ from $\mathrm{C}-\mathrm{H}$ bonds creating subsurface $\mathrm{DBs}$ and $\mathrm{H}$ molecules. $\mathrm{C}$ and hydrocarbon ions penetrate the film (subplantation) in a typical way of displacing $\mathrm{H}$ from $\mathrm{C}-\mathrm{H}$ bonds. $\mathrm{H}$ ions penetrate deepest into the film because they interact weakly with $\mathrm{C}$ atoms due to 
their low mass. So in a complete model of a-C:H film growth, the chemical processes of neutral species and dehydrogenation as well as the physical process of subplantation should be all involved (Robertson, 2002).

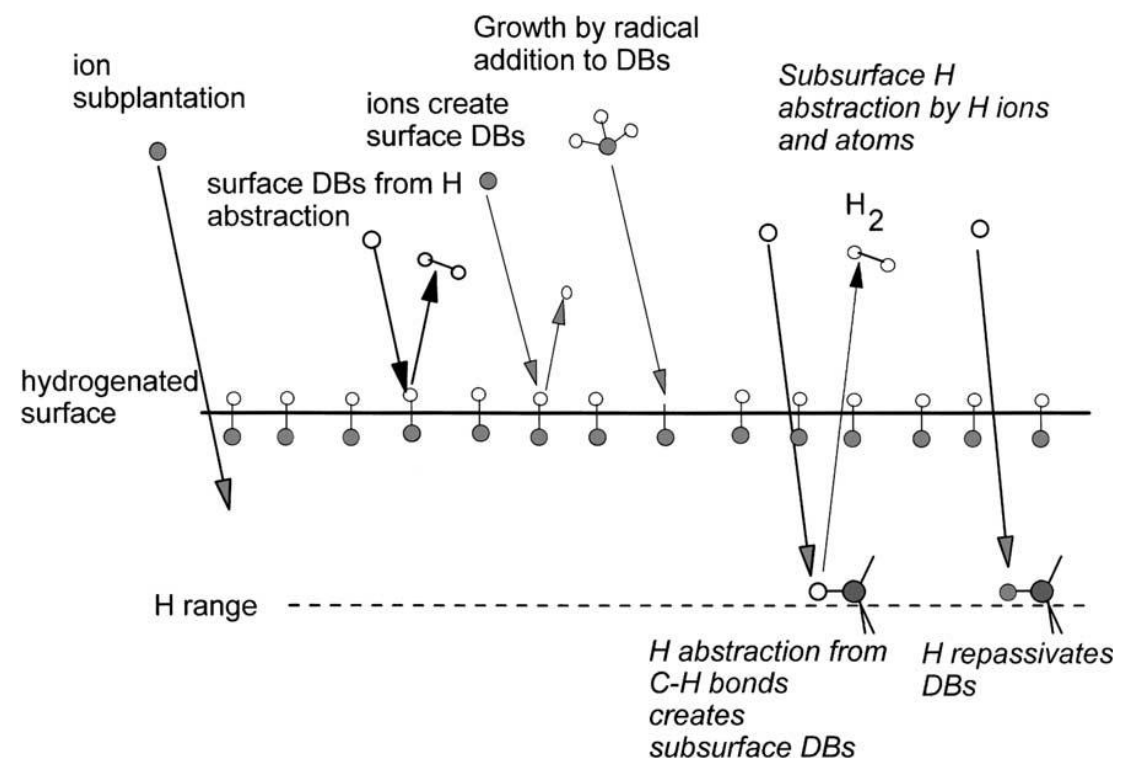

Figure 2.6 Component processes in a-C:H film growth (Robertson, 2002)

Ion energy is a key parameter to determine $\mathrm{sp}^{3}$ fraction in $\mathrm{a}-\mathrm{C}: \mathrm{H}$ films as well as film properties. At low impact energies, normally polymer-like carbon films with predominant $=\mathrm{CH}_{2}$ groups in structure are obtained as the precursor gases may not be sufficiently decomposed. At intermediate impact energies, diamond-like carbon films can be obtained based on the reduction of hydrogen content and the formation of $\mathrm{sp}^{3}$ bonding. If the impact energy is too high, graphite-like carbon films can be obtained because the high energy induces an increase in disordered $\mathrm{sp}^{2}$ bonding. The substrate temperature is also a key parameter for film deposition. When the substrate temperature is above a transition temperature, it's observed that there is a noticeable decrease in the density and $\mathrm{sp}^{3}$ fraction, and the transition temperature decreases with the increasing ion energy during film growth (Erdemir et al., 2006). 


\subsubsection{DLC Deposition Techniques}

Hard and transparent DLC thin films were first synthesized in 1971 by Aisenberg and Chabot using ion beam deposition (non-mass separated $40 \mathrm{eV}$ carbon ions). This work initiated the extensive research and development of carbon-based hard films. A variety of CVD and physical vapor deposition (PVD) methods have been developed for the production of DLC films with a wide range of properties to meet the demands of various applications. For example, the deposition methods have been developed to grow the DLC films with a fraction of $\mathrm{sp}^{3} \mathrm{C}-\mathrm{C}$ bonding up to $90 \%$ and properties similar to diamond.

The main deposition techniques to prepare hydrogen free DLC films include direct ion beam deposition, mass-selected ion beam deposition (MSIBD), ion assisted evaporation, pulsed laser deposition, sputtering, filtered cathodic arc deposition and arc discharge (Lifshitz, 1999). Those deposition techniques can also be used to produce hydrogenated DLC films by changing carbon source from pure carbon to hydrocarbon gases. In addition to those deposition techniques, plasma enhanced CVD has been widely used to produce hydrogenated DLC. Figure 2.7 schematically shows some deposition methods. Each of the deposition techniques has its own advantages and disadvantages in terms of deposition rate, film quality, and uniformity. The deposited DLC thin films can have a wide range of structures and properties that stem from various deposition conditions and parameters in a specific deposition scheme, which include distribution of energetic species involved, energy and angle incidence, ambient pressure during deposition, substrate temperature and deposition rate (Lifshitz, 1999). 
(a) lon beam deposition

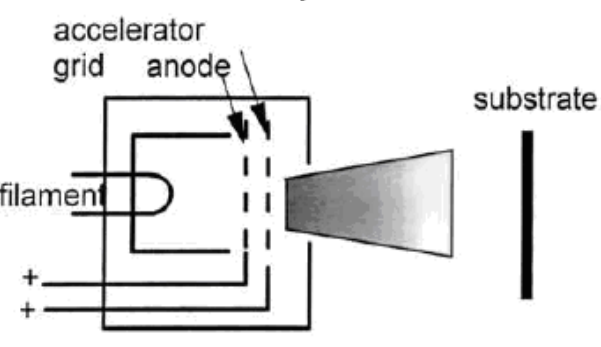

(c) Magnetron sputtering

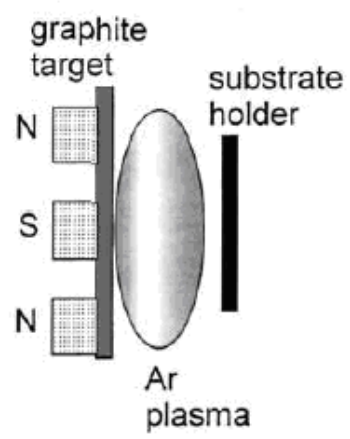

(e) Plasma enhanced CVD

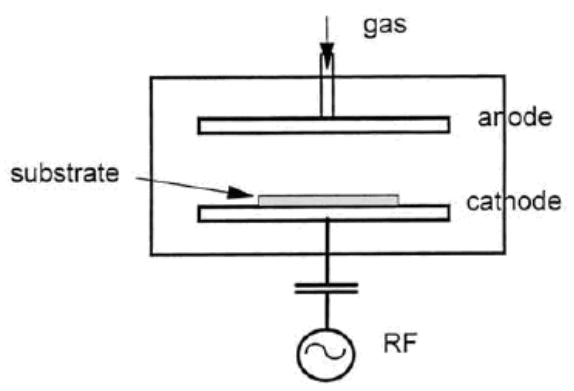

(b) lon beam sputtering

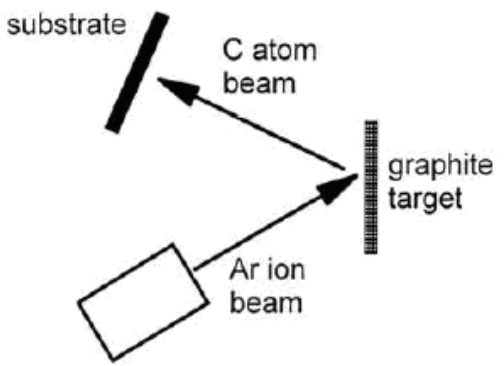

(d) Cathodic Vacuum Arc substrate

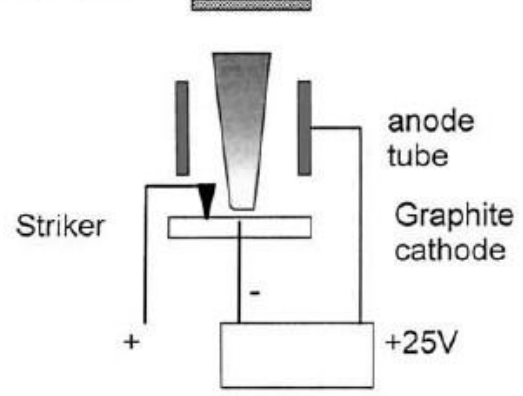

(f) Pulsed laser deposition

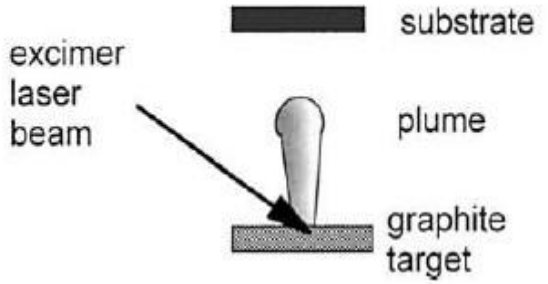

Figure 2.7 Schematics of various DLC deposition methods (Robertson, 2002)

In the family of DLC deposition technique, sputtering is the most common industrial process to produce both hydrogen free and hydrogenated DLC films because of its versatility, wide range of used sputtering materials as well as ease of scale-up (Robertson, 2002). In a sputtering process, energetic ions from radio frequency or direct current plasma are used to sputter carbon species from a carbon target such as graphite, glassy carbon (C) or C-C composites for deposition of a-C thin films (Lifshitz, 1999). Sputtering has many variants, mainly including magnetron sputtering, 
ion beam sputtering, and reactive sputtering. Magnetron sputtering has been used to increase the deposition rate where magnets are placed behind the target to cause the electrons to spiral with their path length increase thus to increase the plasma ionization degree. In recent years, closed-field unbalanced magnetron sputtering has been widely applied with increased deposition rates especially for the production of super-dense, ultra-thin DLC films (Erdemir et al., 2006). Ion beam sputtering can apply a beam of Ar ions to sputter carbon species from a carbon target for deposition of DLC films. Its principal drawback is that a lot of maintenance is required to keep the ion source working (Wolf, 1995). Reactive sputtering can grow a-C:H films by using a plasma of Argon (Ar) and $\mathrm{H}$ or $\mathrm{CH}_{4}$, and produce amorphous carbon nitride $\left(\mathrm{CN}_{\mathrm{x}}\right)$ films by using an Ar-N plasma (Robertson, 2002). In addition, sputtering is very useful for direct investigation of the effects of incorporated foreign elements on the DLC film properties (Nakazawa et al., 2010). Sputtering does not produce the hardest DLC films due to its relatively high ratio of neutral species to energetic ions, however, a relatively high $\mathrm{sp}^{3}$ content can be obtained in DLC films by developing sputtering with a very high fraction of ions, but at the cost of a low growth rate (Robertson, 2002).

Ion beam deposition has been widely used to synthesize DLC thin films due to its ability of independent control of ion energy and ion current density (Tang et al., 2011b). In this technique, the precursor ions (carbon or hydrocarbon ions) are extracted from a plasma to form an ion beam toward the substrate to form films in a high vacuum deposition chamber. Ion beam deposition can be considered as ion implantation where bombarding ions penetrate to subsurface layers and bond with target atoms to grow high quality films (He et al., 1996). The carbon source used in ion beam deposition can be solid carbon target or hydrocarbon gas. 


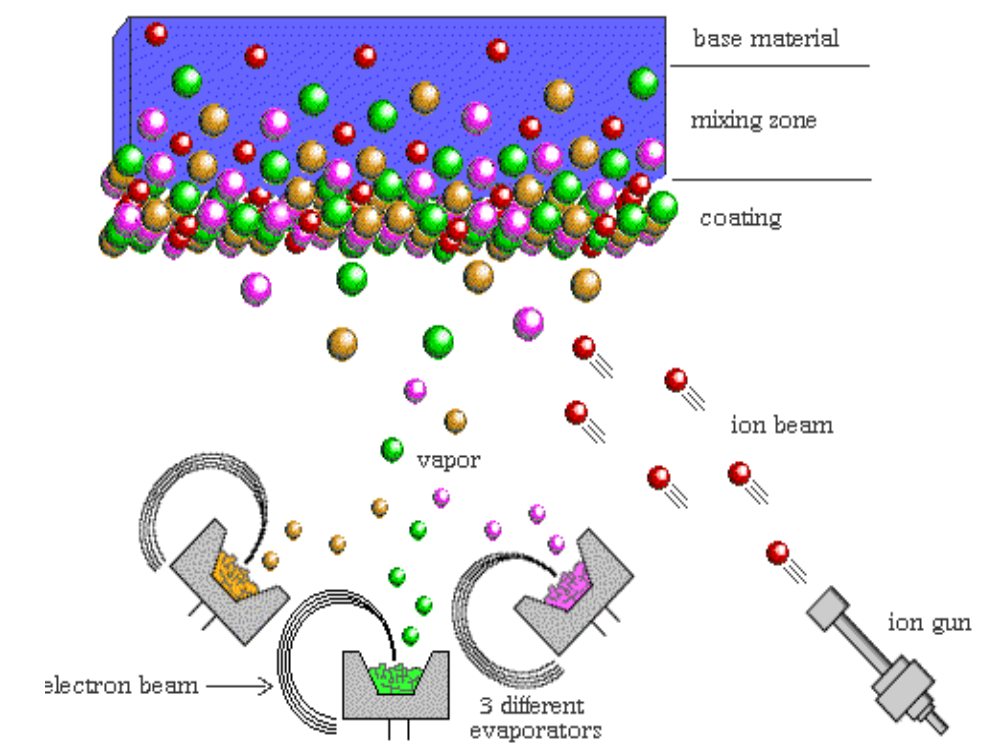

(a)

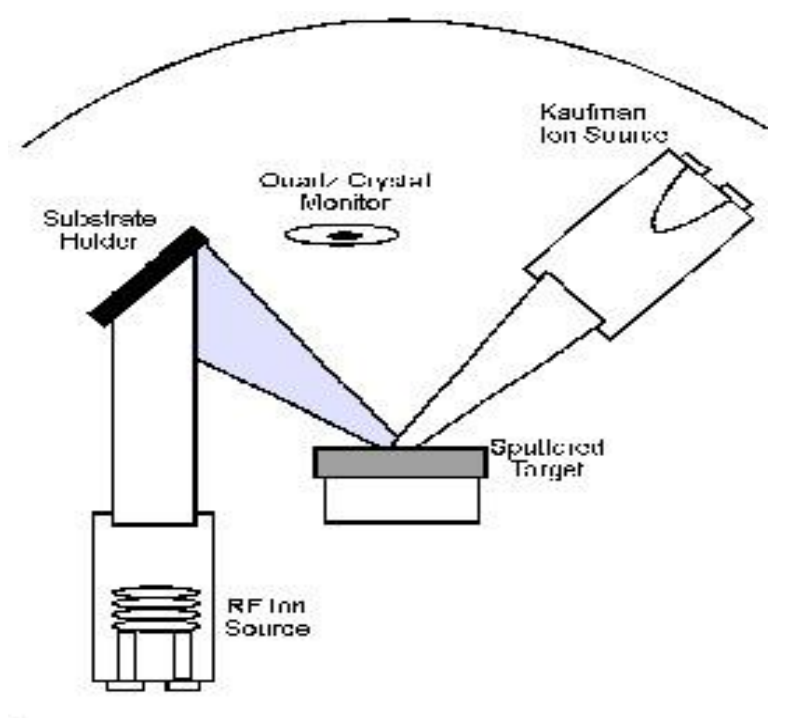

(b)

Figure 2.8 (a) Ion beam assisted electron beam evaporation

(http://cim.aamu.edu/Activities/ibad.html, 2012-1-20); (b) Dual ion beam deposition

(http://web.mit.edu/cprl/www/dualion1.gif, 2012-1-20)

Ion beam assisted deposition is a technique combining ion implantation with simultaneous PVD, e.g. electron beam evaporation or sputtering. Figure 2.8 gives schematics of two different types of ion beam assisted deposition method. Independent control of deposition parameters including ion energy, temperature and ion current density is also available in ion beam assisted 
deposition. Using this technique a gradual transition between substrate and produced film can be created and less built-in strain in the deposited film can be realized, which leads to a much more durable film adhesion to the substrate.

MSIBD, a special case of direct ion beam deposition, is capable of growing high quality DLC films, although this technique is not expected to gain commercial application. It is mostly used for laboratory work and offers controllable deposition species, ion energies as well as an ability of doping by switching the ion species (Robertson, 2002). MSIBD has been considered as the best technique for controlled studies on structures and properties of DLC thin films as well as for fine tuning of films properties (Lifshitz, 1999).

Pulsed laser deposition is a PVD technique. In a vacuum chamber, a pulsed laser beam of high power is applied to strike a target to vaporize its material into a plasma plume (including atoms, molecules, electrons, ions, etc.) for deposition of a thin film on a substrate. Pulsed laser deposition has been successfully used to produce high quality DLC films. It is considered as a versatile laboratory scale method with limited industrial applications. Plasma enhanced deposition uses a glow discharge to activate various hydrocarbon gases needed for DLC deposition in PVD or CVD systems such as methane, ethane, acetylene, benzene, ethylene and propane. Filtered cathodic arc deposition is a very promising technique for large-scale industrial production because of its advantages of producing highly ionized plasma with energetic species having a fairly narrow ion energy distribution and high growth rates for a low capital cost (Robertson, 2002). Cathodic arc systems can achieve high ion current densities and straightforwardly scale up to large areas. The disadvantages of this method are unstable cathode spot and insufficient filtering for some applications. 


\subsection{Doped DLC Thin Films}

The amorphous nature of DLC thin films makes them feasible to be doped or incorporated with various foreign elements. In order to reduce the high compressive stress induced by energetic bombardment during the deposition and to tune the properties of DLC to meet the demands for diverse applications, incorporation of various transition metals, such as Ti, Co, W, $\mathrm{Ni}$, and non-mental elements, such as F, Si, N, B, into DLC films has been recently attracted significant attentions from both scientific and industrial communities.

\subsubsection{Metal Doped DLC}

Doping DLC with metal elements Ti, Co, W, Ni, chromium (Cr), molybdenum (Mo) has been realized using different deposition methods, including biased target ion beam deposition (Tang et al., 2011a ; VijaiBharathy et al., 2011), reactive sputtering (Podgornik et al., 2005), cathodic arc evaporation (Wang et al., 2000), ion implantation (Liu et al., 2007), and dual plasma deposition (Fu et al., 2005). Improved film properties have been obtained from the incorporation of metal into DLC in the form of metal nano-clusters or metallic carbides. Doping Ti and W to DLC was proved to provide better tribological properties under lubricated sliding conditions, especially with the incorporation of $\mathrm{W}$, Podgornik et al. reported up to $50 \%$ reduction in film friction (Podgornik et al., 2005; Vercammen et al., 2004). The metal (Mo and W) doped hydrogenated DLC can exhibit high thermal stability up to an annealing temperature of $500{ }^{\circ} \mathrm{C}$ without oxidation occurring in the film (Fu et al., 2005). Comparing to pure DLC, the metal doped DLC can have higher hardness and elastic modulus, lower friction coefficient and better wear resistance because of the formation of metallic carbides in the films, and film adhesion to substrate can be improved due to interface mixing (Liu et al., 2007; Dai et al., 2007). The metal 
doped DLC thin films have been successfully used on various tools, molds, and dies to prolong their life time.

\subsubsection{Non-metal Doped DLC}

DLC thin films with non-metal elements like N, B, Si, F incorporated have been developed using various deposition techniques, including pulsed laser deposition (Kautek et al., 1996), filtered cathodic arc deposition (Monteiro, 2001), biased target ion beam deposition (Sethuraman, 2009), direct current magnetron sputtering (Kahn et al., 2007), radio frequency plasma enhanced CVD (Hasebe et al., 2007) and plasma immersion ion implantation and deposition (Yao et al., 2004). The non-metal element doping into DLC provides improved film mechanical, tribological, thermal, electronic and biological properties.

It has been reported that doping element of Si or B into DLC leads to decrease of stress level in the films, and increase of film thermal stability (Monteiro, 2001). Adding Si to hydrogenated DLC also improves friction and wear properties (Robertson, 2002; Vercammen et al., 2004). N doping to hydrogenated DLC increases film hardness and adhesion strength, reduces film roughness and improves film haemocompatibility (Sethuraman, 2009). In addition, N doped DLC can exhibit promoted tribological properties (Yan et al., 2004) and field emission properties (Park et al., 1996). F doped DLC thin films possess enhanced electron field emission properties for promising applications of low-threshold field emitters (Ahmed et al., 2007), and possess significantly improved film antithrombogenicity for promising applications on blood contacting medical materials (Hasebe et al., 2007). Addition of F in DLC thin films also leads to fairly good film adhesion to substrate, reduces film roughness and evidently improves film hydrophobicity (Yao et al., 2004). More details about B doping to DLC will be given in the following section. 


\subsubsection{Boron Doped DLC}

Various DLC deposition techniques have been developed to prepare B doped DLC thin films. These include MSIBD via alternating direct deposition of low energy mass-separated C ions and B ions (Ronning et al., 1995), pulsed laser deposition using a XeCl excimer laser of $308 \mathrm{~nm}$ wavelength and polycrystalline graphite and boron carbide targets (Kautek et al., 1996), filtered cathodic arc deposition with a mixed plasma stream from a $\mathrm{B}_{4} \mathrm{C}$ cathode and a graphite cathode as sources (Monteiro, 2001), non-balanced reactive magnetron direct current sputtering using $\mathrm{B}_{4} \mathrm{C}$ target as B source (Ahmad et al., 2007), radio frequency plasma enhanced CVD with $\mathrm{CH}_{4}$ and borane as source gases (Wang et al., 2010), radio frequency magnetron sputtering using a composite target consisting of graphite and boron (Pu et al., 2010), femtosecond pulsed laser ablation with graphite and boron targets alternatively ablated by an amplified Ti:sapphire laser of $800 \mathrm{~nm}$ wavelength (Khadro et al., 2011). The deposition methods and parameters have significant effects on the structure and properties of the synthesized B-DLC films.

It has been reported that B doping into DLC leads to an increased electrical conductivity and B doping is more efficient in increasing film electrical conductivity for electronic applications (emission devices) than $\mathrm{N}$ doping (Ronning et al., 1995; Sikora et al., 2009b). B incorporation to hydrogenated DLC can reduce film stress and promote the formation of $\mathrm{sp}^{3}$ carbon bonding in the films, thus enhance the film hardness and film adhesion strength (He et al., 2000; Ahmad et al., 2007). Similarly, B incorporation in low concentrations to hydrogen free DLC can also result in an increase of $\mathrm{sp}^{3}$ carbon bonding in the films (Monteiro, 2001). However, according to what $\mathrm{Pu}$ et al. reported, B incorporation (up to 26.9 at.\%) to hydrogen free DLC films by radio frequency magnetron sputtering decreases $\mathrm{sp}^{3}$ carbon bonding in the films and $\mathrm{B}$ atoms facilitate 
carbon atoms to form $\mathrm{sp}^{2}$ bonding and graphitization (Pu et al., 2010). Further research work is necessary in order to clarify the effect of B on B-DLC films.

B-DLC thin films have also been deposited on various substrates for their potential applications. For example, B incorporated hydrogenated DLC films coated on grade 316L stainless steel and grade 5 titanium alloy (Ti-6Al-4V) show increased film adhesion strength, demonstrating potentials to be used as protective coatings for biomedical implants (Ahmad et al., 2007). B-DLC thin films on silicon oxide and silicon nitride substrates were used as working electrodes for electrochemical detection of trace heavy metals, and the results show that B doping can enhance the electrode detection sensitivity (Khadro et al., 2011).

In addition, co-incorporation of $\mathrm{B}$ and $\mathrm{N}$ to DLC has been studied with the expectation that the synthesized films may exhibit excellent properties of both B incorporated DLC and N incorporated DLC (Nakazawa et al., 2010). B and N co-incorporated DLC thin films have been fabricated by various deposition techniques, including radio frequency magnetron sputtering (Nakazawa et al., 2010), microwave plasma enhanced CVD (Wu et al., 2002), ion beam assisted deposition (Yasui et al., 2000), and dual cesium ion beam sputtering (Byon et al., 2003). The compounds of boron nitride, $\mathrm{CN}_{\mathrm{x}}$ and boron carbon nitride can be formed in $\mathrm{B}$ and $\mathrm{N}$ co-incorporated DLC films (Ulrich et al., 2009) and the films can exhibit prominent characteristics such as improved tribological properties and toughness (Robertson, 2002), enhanced thermal and chemical stability (Maitz et al., 2006), good thermal conductivity and oxidation resistance (Ulrich et al., 2009).

However, the conventional deposition techniques used to synthesize B-DLC films, such as magnetron sputtering, plasma enhanced CVD and pulsed laser deposition, often suffer from their limitations like high substrate temperature, sophisticated deposition and doping parameters, and 
it's difficult to use them to prepare high performance films for advanced applications. For instance, the widely used magnetron sputtering method is difficult to produce ultra-smooth DLC for magnetic storage application or low contamination DLC for high speed microelectronics. Therefore, there is a need to develop new deposition methods that could synthesize B-DLC films with higher quality and performance. In addition, the structure of B-DLC and its effect on the film properties need to be further investigated and understood.

\subsection{Biased Target Ion Beam Deposition of DLC Based Thin Films}

$\mathrm{BTIBD}$ is a recently developed new technique. It was developed to overcome some drawbacks of conventional magnetron and ion beam sputtering deposition methods to produce high quality thin films. It employs an unfocused, low energy ion source and a negatively biased target for sputtering. It can be operated over a wide range of pressures ( 0.1 to $5 \mathrm{mTorr})$. In a BTIBD system, the ions generated from a low energy broad ion source with typical energies below the sputter threshold of vacuum hardware materials arrive at the target to cause sputtering for deposition, and meantime a second ion source can be directed to the substrate to provide ion bombardment for modification of film structure or direct ion beam deposition. The BTIBD technique is a hybrid combining the advantages of the ion beam deposition and ion beam sputtering techniques (Hylton et al., 2000). It is viewed as a simpler and more reliable alternative to the conventional ion beam deposition employing gridded ion sources. This method offers fine control of adatom energies, excellent uniformity and repeatability. In addition, compared with the traditional ion beam sputtering techniques the BTIBD can realize deposition over larger areas and has the potential of scaling up for industrial applications (Hylton et al., 2000).

BTIBD technique has been used to grow thin, smooth, uniform and high quality DLC based thin films, where one ion source was used to deposit DLC and meantime a second ion source was 
used to sputter a biased target for the incorporation of foreign element(s) to DLC. It has been reported that Ni incorporated DLC (VijaiBharathy et al., 2010b), Ti incorporated DLC (VijaiBharathy et al., 2010a), W incorporated DLC (VijaiBharathy et al., 2011) and Co incorporated DLC (Tang et al., 2011a) have been synthesized using the BTIBD technique, and the synthesized films with metallic carbide nanoclusters formed and uniformly embedded in the DLC matrix exhibit improved properties including excellent biocompatibility, very low friction coefficient and increased adhesion to substrate. However, no work has been reported on the synthesis of B-DLC films using BTIBD and the investigation of their microstructure and mechanical properties. In this work, we report the first results in this field accordingly.

\subsection{Characterization of DLC Thin Films}

\subsubsection{Scanning Electron Microscopy}

SEM is one of the most commonly used techniques to reveal surface morphology or texture. Chemical composition can also be obtained when a SEM machine is equipped with an X-ray spectrometer. In SEM, specimens are scanned by a high energy electron beam in a raster pattern where electrons interact with the specimen atoms at or near specimen surface with signals produced. The generated signals include X-rays, secondary electrons, back-scattered electrons (BSE), transmitted electrons, etc. Normally a single SEM machine does not detect all possible signals. Depending on the types of detected signals SEM works in different modes.

Among all the possible signals, secondary electron is the most commonly detected type in all SEM machines. So the secondary electron imaging mode is the most common or standard SEM detection mode. Secondary electrons are ejected from the specimen atoms near (a few nanometers under) specimen surface by inelastic scattering with the incident electrons. They are detected and used to give accurate topographic information of the specimens. In the secondary 
electron imaging mode, SEM can produce images of specimen surface with very high resolution, revealing details within a few nanometers in size. SEM also works in the BSE mode where back-scattered electrons are detected. Back-scattered electrons refer to the beam electrons that are reflected from specimens by elastic scattering interactions with specimen atomic nucleus or outer shell electrons. Since BSE signal intensity strongly depends on the atomic numbers of specimens, SEM in the BSE mode can produce images of specimens with information of the distribution of different elements in the specimens.

Figure 2.9 shows a schematic of a typical SEM system. In SEM, a specimen in an appropriate size mounted on a specimen stub is placed into a high vacuum chamber. The specimen is required to be electrically conductive or with electrically conductive coating, so it can be grounded to prevent electrostatic charge accumulation at the surface which may lead to scanning faults and image artifacts. The electrons are emitted thermionically from a tungsten filament cathode fitted in an electron gun or produced by field emission, and then accelerated and focused to a beam by electrical and magnetic field optics. The electron beam in a typical energy range of $0.2 \mathrm{keV}$ to $40 \mathrm{keV}$ passes through scanning coils in the electron column and generates scans in a raster pattern over the specimen surface. From the electron beam interaction with the specimen surface, the signals of secondary electrons, BSE, X-rays are produced and each of them can be detected by specialized detectors. Those signals are amplified by various electronic amplifiers and then displayed and recorded in a computer terminal. The resulting SEM images are actually distribution maps of the signal intensities, providing details of the scanned area of the specimen. 


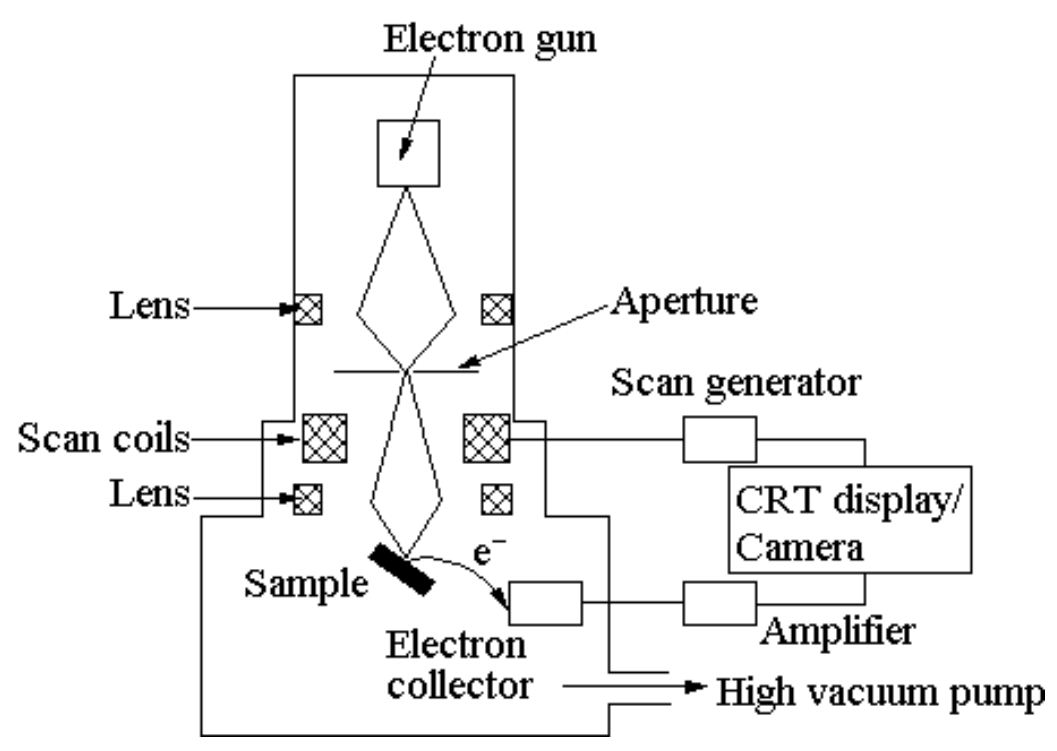

Figure 2.9 Schematic of SEM (http://www.chm.bris.ac.uk/pt/diamond/stuthesis/chapter2.htm,

One of the main features SEM has is its large depth of focus comparing to other types of microscopy, which makes it possible to image very rough surfaces. SEM is a non-destructive analysis technique as electron-material interactions do not lead to specimen volume loss, so the same specimen can be analyzed repeatedly. A wide range of magnifications can be obtained in SEM, from about 10 times to more than 500,000 times. The resolution of SEM images can reach to a few nanometers. Also, it's comparatively easy to operate SEM with user-friendly intuitive interfaces and data acquisition is rapid. However, very light elements such as $\mathrm{H}$, helium and lithium cannot be detected using X-ray spectrometer in SEM and SEM requiring specimens to be solid, electrically conductive or with electrically conductive coating, and stable in a vacuum chamber might result in limited uses. For characterization of DLC thin films, SEM is not very powerful because the films show ultra-smooth surfaces without any special features. 


\subsubsection{Atomic Force Microscopy}

AFM is a commonly used technique to obtain three-dimensional surface topography. It is a type of scanning probe microscopy with resolution down to fractions of a nanometer. AFM can work under an ambient environment or even a liquid environment for imaging and measuring at nanoscale. Comparing to SEM, AFM has a higher resolution. For characterization of DLC thin films showing ultra-smooth surfaces, AFM is a powerful technique to observe and accurately measure their surface morphology (Lifshitz, 1999).

Figure 2.10 shows a schematic of a typical atomic force microscope. In AFM, a cantilever with a sharp tip, i.e. a probe is applied to scan specimen surface in close proximity. The probe does not scan at constant height due to the risk of damages caused by collisions between the probe and the specimen surface. In modern atomic force microscopes, a vertical piezoelectric scanner is employed to adjust the distance between the probe and the specimen surface based on a feedback mechanism to keep the force between them constant. The force can be mechanical contact force, capillary force, chemical bonding, magnetic forces, etc. The constant force makes the probe deflect and the deflections are measured by a laser spot reflected from the probe top surface to photodiodes and collected by a position-sensitive detector. Upon the probe scanning across the specimen surface, both the probe vertical displacements realized by the piezoelectric scanner to maintain the constant force and the probe deflections caused by the force are recorded to represent specimen topography. 


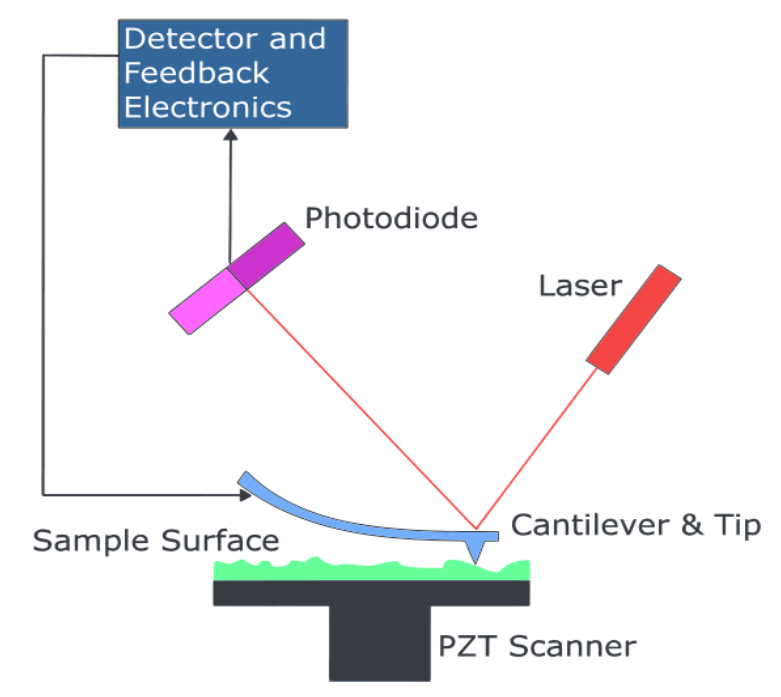

Figure 2.10 Schematic of AFM

(http://en.wikipedia.org/wiki/File:Atomic_force_microscope_block_diagram.svg, 2012-2-8)

Different operation modes have been developed for different AFM applications. The primary operation modes are static mode and dynamic mode. The static mode is also called contact mode where surface contours are measured and imaged based on the soft physical contact between probe and specimen surface, and the collection of probe deflections and vertical displacements. This mode is commonly used for rigid surfaces. The dynamic mode includes non-contact mode and tapping mode (or intermittent contact mode). In the dynamic mode, a probe is in external oscillation (close to its resonance frequency) and with the probe getting close to the specimen surface the interaction force between them changes the probe oscillation frequency or amplitude. The probe-to-surface distance is adjusted to maintain a set probe oscillation amplitude or frequency and then recorded at each $(\mathrm{x}, \mathrm{y})$ data to describe specimen characteristics. The non-contact mode works well to measure soft specimens and rigid specimens with a few monolayers of adsorbed fluid on surface. The tapping mode generates higher lateral resolution than the contact mode and it is widely used to image biological specimens. 
One of the main features of AFM is its ability to provide three-dimensional surface topography with true atomic resolution on both conductors and insulators. However, AFM modes have relatively slow rate of scanning (several minutes for a typical scan), not like SEM scanning in real time. In addition, AFM can only image a maximum height on micrometer order with a maximum scanning area around $150 \times 150 \mathrm{~mm}$, whereas SEM can image an area on millimeter by millimeter order with a depth of field on millimeter order. Due to the probe nature, normally AFM cannot measure steep walls or overhangs.

\subsubsection{X-ray Diffraction}

$\mathrm{XRD}$ is an important non-destructive analytical technique to identify the crystal structure of materials. It has been applied in geology, material science, environmental science, chemistry, pharmaceutical industry, etc. XRD is based on elastic scattering of X-rays from long range order structure.

As Figure 2.11 shows, X-rays scatter when they incident on a crystal lattice. X-ray diffraction occurs when scattering in a certain angle from different crystal planes is in phase. In this condition, the scattered X-rays combine to form new enhanced wave fronts mutually reinforcing each other. This condition is given by Bragg's Law, $2 \mathrm{~d} \sin \theta=\mathrm{m} \lambda$, $\mathrm{d}$ is distance between crystal planes, $\theta$ is $\mathrm{X}$-ray incident angle, $\lambda$ is $\mathrm{X}$-ray wavelength and $\mathrm{m}$ is an integer multiple. Each crystalline material has a characteristic crystal lattice so it diffracts X-rays in a unique pattern. 


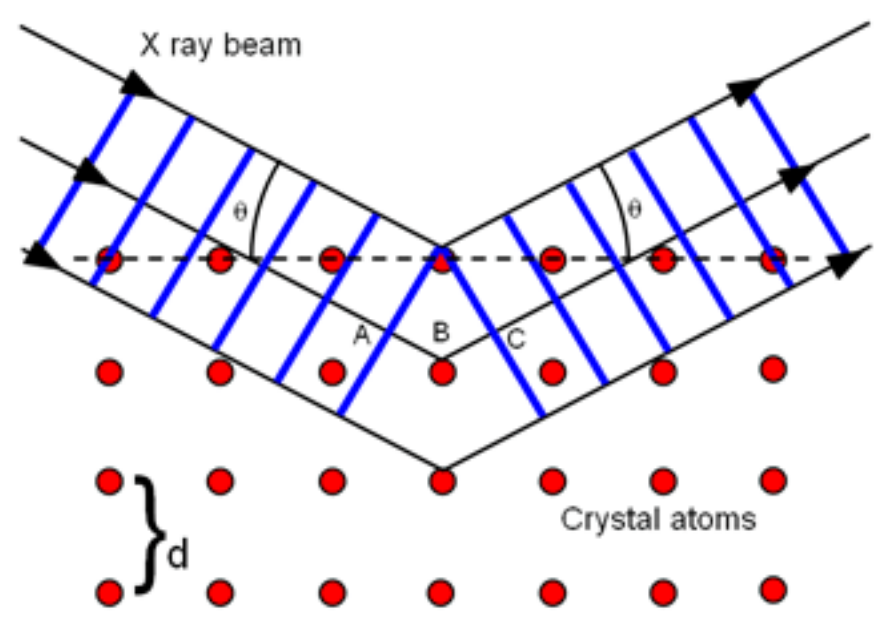

Figure 2.11 Schematic of XRD (http://tap.iop.org/atoms/xray/530/page_47297.html, 2012-2-10)

In a powder X-ray diffractometer, a specimen is mounted on a goniometer and rotates. A beam of monochromatic X-rays is directed to strike the specimen. With the specimen rotating by an angle $\theta$, the $\mathrm{X}$-ray incident angle $\theta$ is realized. The scattered $\mathrm{X}$-rays are produced from the specimen and then land on an X-ray detector passing through divergent slits with radiation collimated and background noise reduced. The X-ray detector coupled with the goniometer also rotates and it rotates by an angle $2 \theta$ when the specimen rotates by $\theta$. The intensities of the scattered X-rays and the angles $2 \theta$ are recorded to create a XRD pattern. Diffraction peaks appear on the XRD pattern at certain angles. Based on the diffraction peaks, the crystal structure of materials can be determined by comparing the diffraction data with the database (maintained by the International Centre for Diffraction Data). Powder XRD can also be used to characterize the crystallite size (grain size) and preferred orientation of polycrystalline or powdered solid materials and to determine their strains. For thin film materials, grazing incidence XRD is usually applied. 


\subsubsection{Raman Spectroscopy}

Raman spectroscopy is a widely used technique to study vibrational, rotational and other low frequency modes in condensed matters and help identify bond types in the matter. It includes illuminating specimen surface with monochromatic light and examining light scattered by the specimen using a spectrometer. Raman spectroscopy is based on the Raman scattering which is the inelastic scattering of incident monochromatic light. In Raman spectroscopy, the photons from an incident monochromatic laser in near infrared or near ultraviolet range interact with molecular vibrations, or other excitations in the specimen. The specimen molecules are excited by the incident photons from the initial state to a virtual energy state, and then relax with scattering the incident photons and finally return to a different vibrational state which can be more energetic or less energetic than the initial state. The energy difference between the incident photons and the scattered photons corresponds to the up- or down-frequency shift which is termed as Raman shift. A plot of detected photon number (scattered light intensity) versus Raman shift is a Raman spectrum. Raman spectra are characteristic because different substances have different vibrational modes. Analyzing and comparing Raman spectra with a library of known spectra, materials can be identified.

Figure 2.12 shows a schematic of a Raman spectrometer. In a modern Raman spectrometer, a highly monochromatic laser is used as a photon source and the laser beam is focused to a small spot by a microscope to illuminate a specimen. A notch or edge filter is employed for laser rejection cutting out the intense elastic Rayleigh scattered laser light/radiation, which is necessary because the Raman scattered component is very weak comparing to the concurrent Rayleigh scattered component. Then the rest inelastic Raman shifted light/radiation is dispersed 
onto a charge-coupled device detector. There is also a computer used for data acquisition and curve fitting.

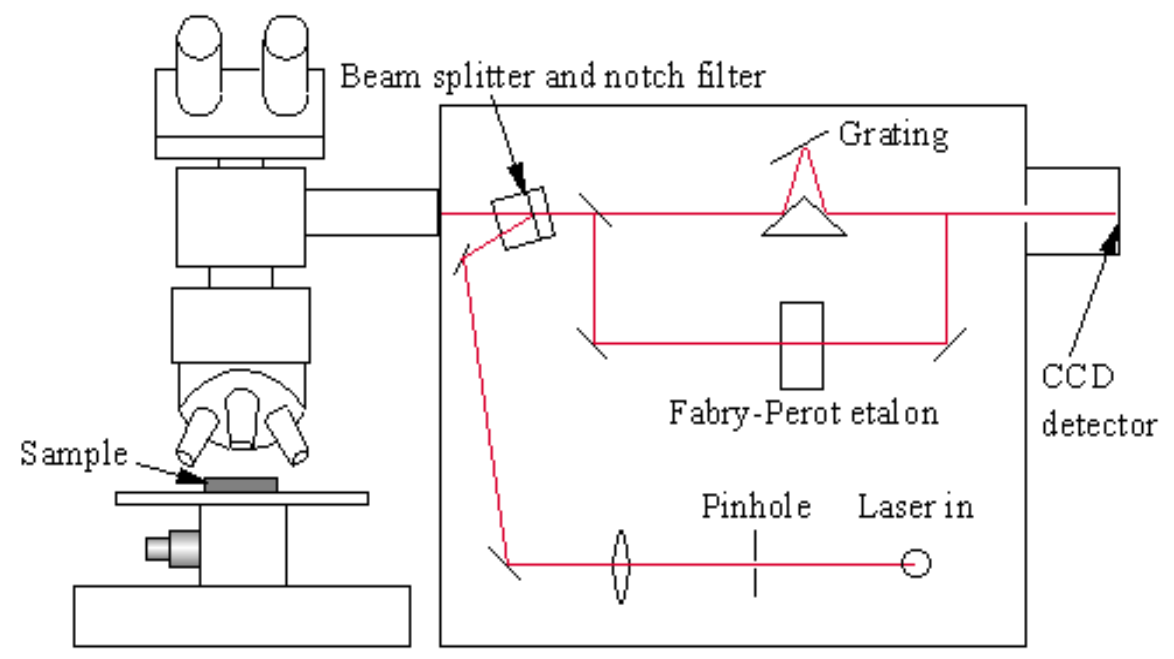

Figure 2.12 Schematic of a Raman spectrometer

(http://www.chm.bris.ac.uk/pt/diamond/stuthesis/chapter2.htm, 2012-2-13)

Typical applications of Raman spectroscopy are structure and substance determination, component qualitative and quantitative analysis. Raman spectroscopy is very sensitive to distinguish carbon $\mathrm{sp}^{2}$ and $\mathrm{sp}^{3}$ bonds so it has been widely used, as a routine and non-destructive way, to detect diverse carbon materials such as diamond, graphite, DLC, carbon nanotubes and fullerenes. For various carbon materials, Raman spectra show different characteristic peaks. Figure 2.13 gives a comparison of Raman spectra which are taken using $514.5 \mathrm{~nm}$ laser radiation. Natural diamond exhibits two characteristic peaks at $1332 \mathrm{~cm}^{-1}$ and $2750 \mathrm{~cm}^{-1}$ in the Raman spectrum. Large single crystal graphite exhibits a single sharp peak at $1580 \mathrm{~cm}^{-1}$ in the Raman spectrum which is labeled with "G" for graphite. For small crystallite size or disordered graphite, the sharp graphite $\mathrm{G}$ peak becomes a broad $\mathrm{G}$ band and a second peak appears at $1350 \mathrm{~cm}^{-1}$ in the Raman spectrum which is labeled with " $\mathrm{D}$ " for disorder. Amorphous carbon exhibits a broad asymmetric hump from 1000 to $1600 \mathrm{~cm}^{-1}$ in the Raman spectrum. DLC thin films show very 
broad D and G bands or peaks in Raman spectra, comparing to the very sharp Raman peaks of diamond and single crystal graphite as well as the relatively broader Raman peaks of microcrystalline graphite. For DLC thin films, it has been reported that the ratio of integral area under $\mathrm{D}$ and $\mathrm{G}$ bands is closely related to the ratio of $\mathrm{sp}^{3}$ to $\mathrm{sp}^{2}$ bond content in the films, and the $\mathrm{sp}^{3}$ bond content in the DLC films increases with the decrease of integral area ratio (Ferrari et al., 2000).

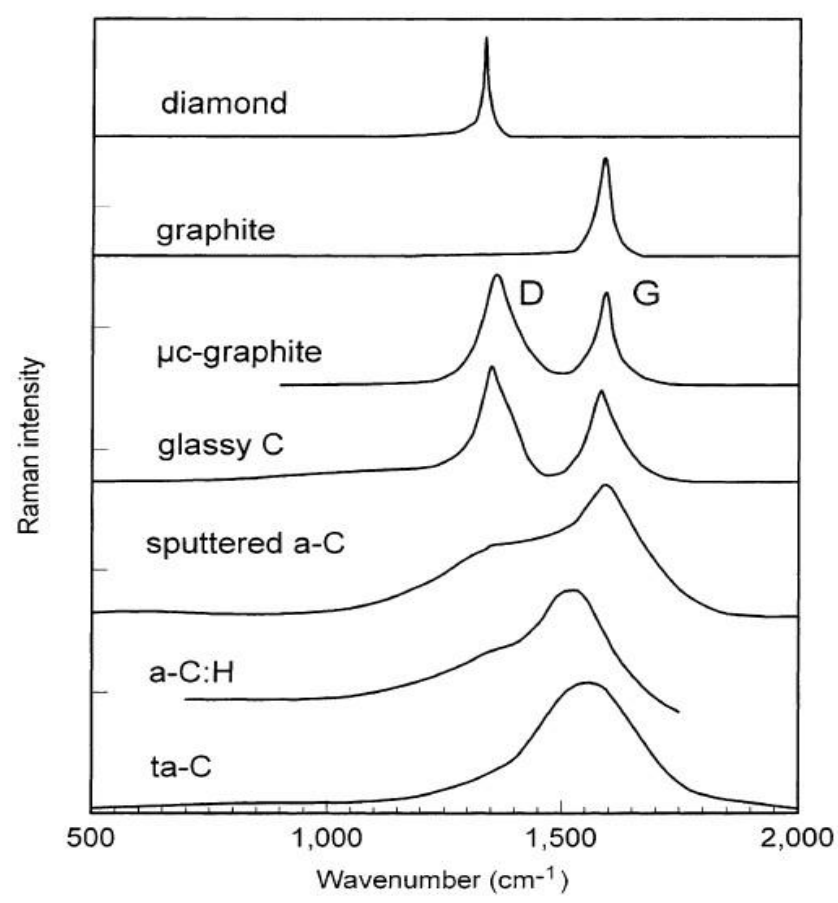

Figure 2.13 Typical Raman spectra of various carbon materials (Robertson, 2002)

Raman spectroscopy has many features and advantages over other analysis techniques, including no destruction to specimens, no need of specimen preparation, no need of vacuum environment, short time scale (acquiring Raman spectra quickly), and its ability to work with solids, liquids or gases as well as glass vials (no interference). However, Raman spectroscopy has relatively low sensitivity making it difficult to measure low concentrations of substances because the Raman effect is very weak. An alternative technique, resonance Raman spectroscopy, can be used to measure low concentrations, which has increased Raman effect. In addition, 
Raman spectroscopy can be used for microscopic examination of minerals, polymers, ceramics, cells, proteins and forensic trace evidence.

\subsubsection{X-ray Photoelectron Spectroscopy}

XPS, also known as electron spectroscopy for chemical analysis, is a quantitative electron spectroscopic technique to determine elemental composition, empirical formula, chemical state and electronic state of the elements existing in a material. XPS is based on the photoelectric effect, i.e. electrons escaping out of a material surface after photon absorption.

In a typical XPS system, a soft X-ray source with photon energy of 1 - $2 \mathrm{keV}$ irradiates a material surface and then the core or inner shell electrons of target atoms are excited to emit photoelectrons by absorption of the energetic X-ray photons. The emitted photoelectrons are detected by an electron energy analyzer, and their kinetic energies and numbers are simultaneously measured. According to Einstein's law, the energy of the absorbed photon is more than the kinetic energy of the emitted free photoelectron and their energy difference is considered as the binding energy of the electron which is characteristic for each element. So the analysis of the measured kinetic energies of the emitted photoelectrons can indicate what elements exist in the material. When the shifts of core levels of elements are measured in a XPS system, XPS provides the information of element chemical bonding states and it is chemically specific because each element has its own core level. Carbon has a small core level shift between

diamond and graphite due to the slightly different bond lengths of $\mathrm{sp}^{2}$ and $\mathrm{sp}^{3}$ bonds (Robertson, 2002).

Normally there are several steps to finalize and analyze a XPS spectrum. The first step is to identify spectral lines and associate them with particular energy levels of corresponding elements. The second step is to calculate photoemission intensities with inelastic scattering background 
eliminated. It's necessary to eliminate this background as the photoelectrons emitted at various subsurface depths experience intensive inelastic scattering before escaping from a material surface. The methods proposed by Shirley, 1972 and Tougaard, 1988 are most commonly used to calculate inelastic scattering background. The third step is correction of XPS peak intensities by the sensitivity factors of atomic energy levels that are instrument-specific and obtainable from standard calibration. After this step it's considered that the intensities of XPS peaks are proportional to the number of atoms in the analyzed volume, thus the calculation for atomic concentrations is straightforward.

XPS is highly surface sensitive and specific. Depending on materials, normally only the photoelectrons emitted from the top 2 to 20 atomic layers can be detected (Tang, 2010). XPS can be used to detect all elements except for $\mathrm{H}$ and helium because regularly used $\mathrm{X}$-ray photon energies are too far from resonance with their ionization potentials, which makes ionization probability very low and results in outgoing signals way below sensitivity thresholds of typical XPS systems. XPS detection limit for most of the elements reaches parts per thousand and it may reach parts per million based on special conditions of concentration at top surface or very long collection time. However, XPS measurements require ultrahigh vacuum conditions and degrade specimens, especially organic chemicals which can be readily degraded by either X-ray photon energy or heat from non-monochromatic X-ray sources. In addition, XPS can be performed with synchrotron radiation sources which provide intense and wavelength tunable X-ray beams.

\subsubsection{Near Edge X-ray Absorption Fine Structure}

NEXAFS is a type of X-ray absorption spectroscopy (XAS) that is widely used to determine local geometric and/or electronic structure of matters in solid, gas phase or solution. NEXAFS is synonymous with X-ray absorption near edge structure (XANES). They are considered as a same 
technique and just different in the used energy range. Conventionally, NEXAFS refers to soft X-ray spectroscopy with photon energy lower than $1000 \mathrm{eV}$ and XANES refers to hard X-ray spectroscopy.

NEXAFS is based on the fundamental phenomenon of X-ray photoabsorption by an atom core level with consequent emission of photoelectrons, fluorescence and Auger electrons. Figure 2.14 gives a schematic of this phenomenon. When an X-ray beam at proper energies incidents on a material surface, inner core level electrons are excited by absorption of X-ray photons to empty states with emission of photoelectrons, and then filling the resulting core holes by electrons from other levels leads to emission of fluorescence or Auger electrons. The electron transition is from a core level or other inner levels to an unoccupied discrete or continuum higher energy state. In NEXAFS the emitted fluorescent photons, Auger electrons and photoelectrons can be measured. The measurement sums over states of the initial core level and all possible photoelectron final states. NEXAFS spectra can be obtained by tuning X-ray photon energy (to a range where core electrons are excited) and collecting data of electron or fluorescence emission. The "name" of absorption edge in NEXAFS measurements depends on the core electron which is excited, for example, the principal quantum number of 1, 2 and 3 corresponds to K-, L- and M-edge, respectively. 


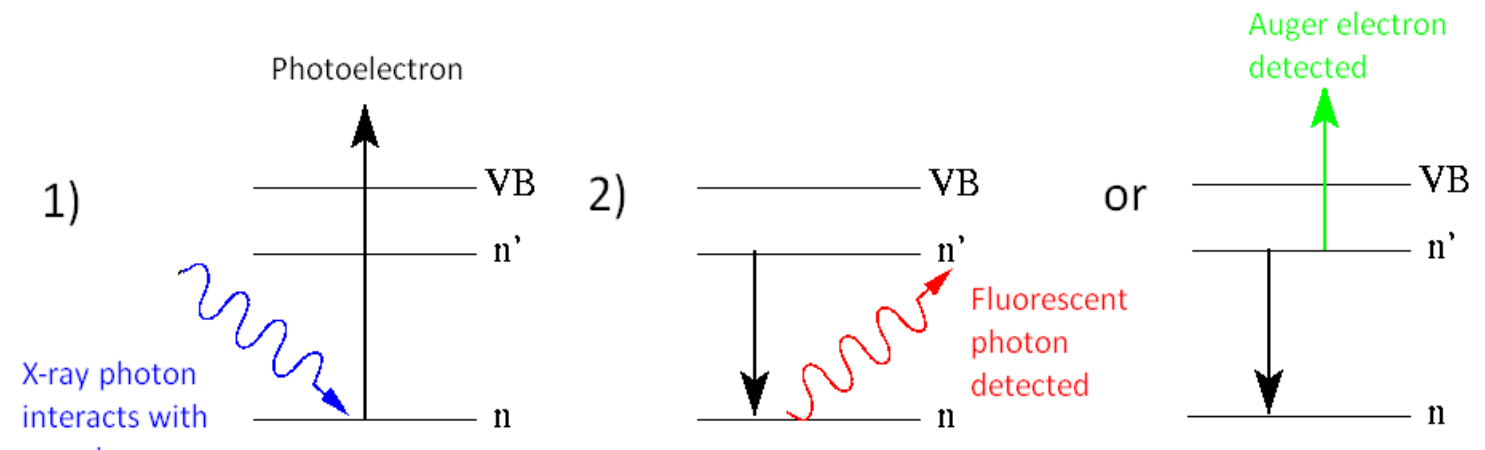

sample

Figure 2.14 Schematic of electron transitions by X-ray incident on a material (https://commons.wikimedia.org/wiki/File:Photoabsorb.png, 2012-2-16)

NEXAFS spectra have a kinetic energy range of up to $50 \mathrm{eV}$ beyond absorption threshold or edge, where multiple photoelectron scattering events dominant. They normally show largest variations in the X-ray absorption coefficient. NEXAFS is element specific as different elements have different and specific X-ray absorption threshold energies. Also, it is very sensitive to chemical states of elements like oxidation state. Therefore, NEXAFS can work in fingerprinting to identify particular chemical species. For DLC thin films, NEXAFS is considered as the most suitable and powerful technique to study film electronic structure and bonding state at an atomic scale. It can be used to distinguish $\mathrm{sp}^{2}$ and $\mathrm{sp}^{3}$ bonding in nanostructured diamond and diamond-like carbon materials and determine $\mathrm{sp}^{2}$ and $\mathrm{sp}^{3}$ bonding concentrations.

NEXAFS spectra are normally collected in two modes. One is fluorescence yield (FLY) mode where the fluorescent photons emitted from samples are measured and their number is proportional to the number of X-ray photons absorbed in samples (Tang et al., 2011a). The other is total electron yield (TEY) mode where the total number of electrons escaping from sample surface is measured including photoelectrons and Auger electrons, and the number is closely related to the X-ray absorption process. FLY is relatively bulk sensitive, for example, the 
probing depth in FLY measurements for carbon K-edge can reach about $100 \mathrm{~nm}$ (Tang, 2010). TEY is relatively surface sensitive and the probing depth in TEY measurements is only several nanometers. For the same specimen both TEY spectra and FLY spectra can be obtained and analyzed to obtain details of electronic structure.

NEXAFS measurements usually require intense tunable soft X-rays so they are performed with synchrotron radiation sources which provide both intense and wavelength tunable X-ray beams. In a synchrotron, electrons are accelerated linearly or centripetally to a velocity near the light speed and circulate in storage rings with electromagnetic radiation emitted. Bending magnets, undulators and/or wigglers are used to periodically accelerate the electrons and bend their path to be circular. Synchrotron radiation is generated via accelerating ultrarelativistic electrons through magnetic fields and with frequencies generated ranging from microwaves to hard X-rays. Synchrotron radiation also has polarization, high flux/intensity, high brilliance, high collimation and stability. If a synchrotron is with energy of several gigaelectronvolts and radius of several tens of meters, the emitted maximum photon energy may reach the order of $1 \mathrm{keV}$ and the obtained synchrotron spectrum may extend from far infrared to hard X-ray region. For NEXAFS measurements, the synchrotron radiation of soft X-rays travels from the ring in a beam line to the end station where specimens are mounted. Synchrotron-based NEXAFS has been applied more and more widely to explore electronic structure and bonding state of various elements in thin films.

\subsubsection{Nanoindentation Testing}

Nanoindentation is a primary and commonly applied method to measure the mechanical properties of DLC thin films as well as small volumes. In nanoindentation, normally a diamond nanoindenter is forced into and then unloaded from a film under small loads (micro- to 
milli-Newtons) and then the small loads and depths of penetration (the indentation profile) or displacements are recorded with high accuracy and precision. The recorded data can be used to calculate and determine elastic modulus, hardness, yield strength and fracture toughness of a surface layer or thin film based on different calculation methodologies. Nanoindenter is a main component used in nanoindentation. There are many types of nanoindenters with different tip geometries, and some of them such as Berkovich, cube corner, Vickers, and Knoop nanoindenters have become a well-established common standard for nanoindentation testing due to their extended use and well-known properties.

The hardness measurements for thin films by nanoindentation are easily affected by many factors like substrate and film surface roughness. The hardness value of the same thin film usually varies depending on different calculation methods, so it's important to present the calculation method when presenting the hardness value, such as the Oliver and Pharr method, the deformation energy method and the force indentation function method. Currently software is used as the best method to collect measurement data and analyze curves of nanoindentation load versus displacement for the calculation of hardness and elastic modulus. For example, the Martens hardness, HM, is relatively simple software which starts with searching for the maximum displacement point $\mathrm{h}_{\max }$ and maximum load $\mathrm{P}_{\max }$. The hardness is given by $H M=\frac{P_{\max }}{A_{s}} \quad(\mathrm{http}: / /$ en.wikipedia.org/wiki/Nanoindentation, 2012-2-20)

$\mathrm{A}_{\mathrm{s}}$ is the contact surface area depending on nanoindenter geometry and displacement. For a perfect Berkovich nanoindenter,

$$
A_{s}=24.5 h^{2}{ }_{\max } \text { (http://en.wikipedia.org/wiki/Nanoindentation, }
$$


Thus, the hardness can be written as

$H M=0.0408 P_{\max } / h_{\text {max }}^{2}$

Versatile nanoindentation testers have been designed and applied for instrumented nanoindentation testing which has been found advantageous in repeatability and reliability. The Universal Nano+Micro Tester manufactured by CETR, as shown in Figure 2.15, is a novel instrument that can be used for nanoindentation testing to obtain hardness and Young's modulus values of DLC films. Gitis et al. used this instrument to test DLC films and reported that for nanoindentation testing repeatable and substrate-independent hardness and elastic modulus values are available when the indents are under $5-10 \%$ of the total film thickness (Gitis et al., 2008).

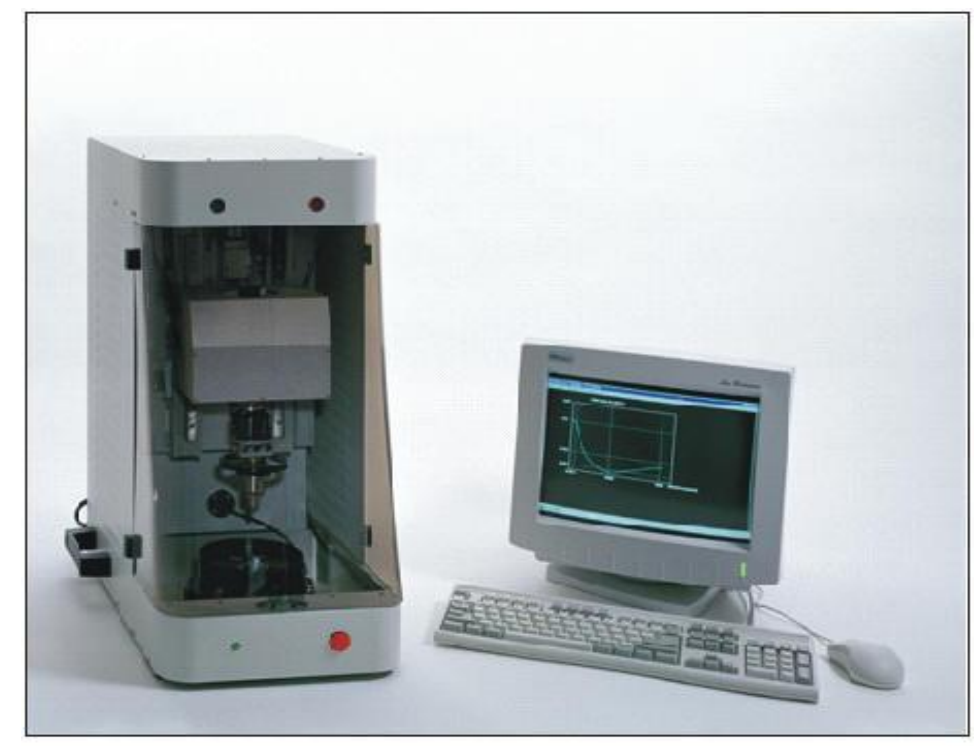

Figure 2.15 Universal Nano+Micro Tester

(http://www.cetr.com/Brochures/Nano-Indentation/determination_of_storage_and_los_moduli_ UNMT.htm, 2012-2-20) 


\subsubsection{Friction Measurement}

Friction generally refers to the resistance to relative motion (like sliding or rolling) or motion tendency of two surfaces or layers which are in contact with each other. Friction force refers to the resistive tangential force acting directly opposite to the direction of motion. During relative motion between two surfaces in contact, friction converts kinetic energy into heat, which can cause dramatic consequences like starting a fire or temperature-caused damage. Also, friction causes wear which may lead to performance degradation and/or surface damage.

There are many types of friction including dry friction (or Coulomb friction), fluid friction, lubricated friction, skin friction and internal friction. Dry friction and fluid friction are the most commonly experienced and investigated types. Dry friction exists when two dry surfaces in contact have relative motion or motion tendency. Fluid friction exists between adjacent or contact layers within a fluid which have relative motion. For dry friction, the friction coefficient is used to describe material friction performance. It is defined as the ratio of friction force to normal force (perpendicular net force pressing two surfaces together). Friction coefficients depend on materials, test conditions and environments, ranging from near zero to bigger than one.

In terms of DLC thin films, friction is mainly dry friction resisting relative motion of two surfaces, including static friction between non-moving surfaces and kinetic friction between moving surfaces. DLC films have good friction performance and the reported friction coefficient values for various DLC films range from 0.001 to 0.7 (Erdemir et al., 2006). The friction between two DLC films depends on film mechanical properties (such as hardness, elastic modulus, fracture toughness), film physical and chemical properties (such as thermal conductivity, surface energy, adsorption characteristics, chemical reactivity), film conditions 
(such as roughness, contact area) and operating conditions (such as load, speed, interface temperature, environment). Friction behavior of DLC thin films is an important factor that can help determine some specific film performance like durability.

Friction testers or tribometers with different configurations and specific simulated contact arrangements, such as block-on-ring, pin-on-disc, ball-on-flat and flat-on-flat, have been applied to evaluate friction behavior of DLC thin films based on the principle of simulating working conditions, measuring and analyzing film regression. For the use of friction testers, mathematical models are established to calculate friction coefficients based on the Coulomb approximation, assuming that two surfaces atomically closely contact only over the contact area which is proportional to the normal force and the friction force is proportional to the normal force. The contact area refers to the real contact happening at the tips of the surface asperities (roughness), and normally it is only a very small fraction of the apparent area. The high stress on the contact area can result in asperity deformation and even adhesive bond deformation or cleavage. The Coulomb approximation is only a rule of thumb simplifying and evaluating outcome of a complicated physical interaction. When using tribometers worn surface morphologies of the thin films under different test conditions can be observed by SEM, AFM, friction force microscope or point contact microscope. Figure 2.16 gives a schematic of pin-on-disc sliding testing. In the pin-on-disc testing, a small load is applied to a stationary pin to keep it in contact with a rotating disc. The tip of the pin is often in spherical shape to simplify the contact geometry for calculation. Friction forces and loading forces are automatically measured and recorded. Friction coefficients are determined by the ratio of frictional force to applied loading force. Pin-on-disc testers work well on conducting a simple friction and wear test for DLC films. 


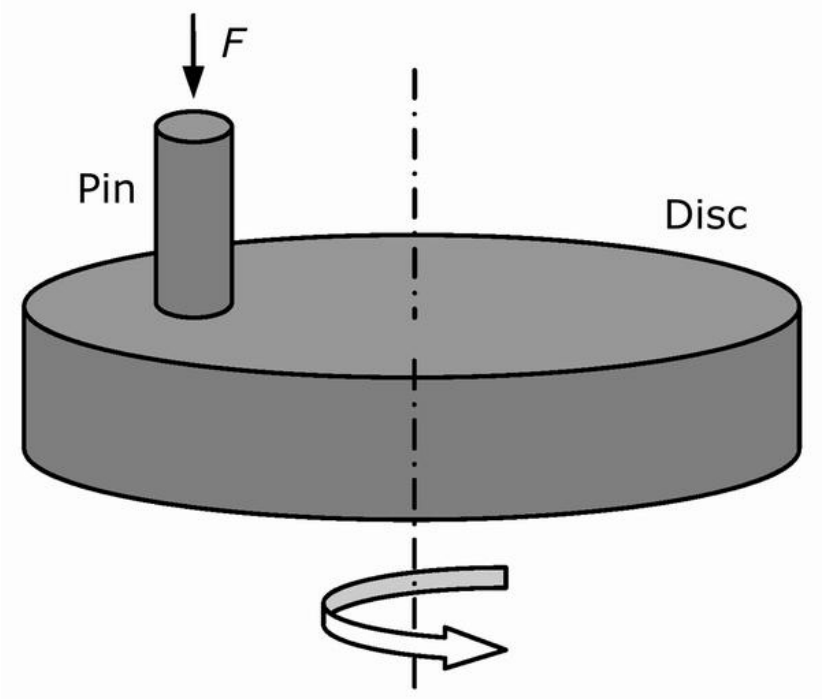

Figure 2.16 Schematic of pin-on-disc sliding testing (http://tribolab.mas.bg.ac.rs/english/images/equipment/T-62_sketch.jpg, 2012-2-22) 


\section{CHAPTER 3 \\ EXPERIMENTAL DETAILS}

\subsection{Biased Target Ion Beam Deposition System}

The thin film samples for the present thesis research work were prepared using the BTIBD system at the Department of Mechanical Engineering, University of Saskatchewan. This system was custom designed and manufactured by 4 Wave Inc. It consists of a high vacuum chamber, a pumping system, a power supply, and a computer control system. Figure 3.1 shows a picture of the system.

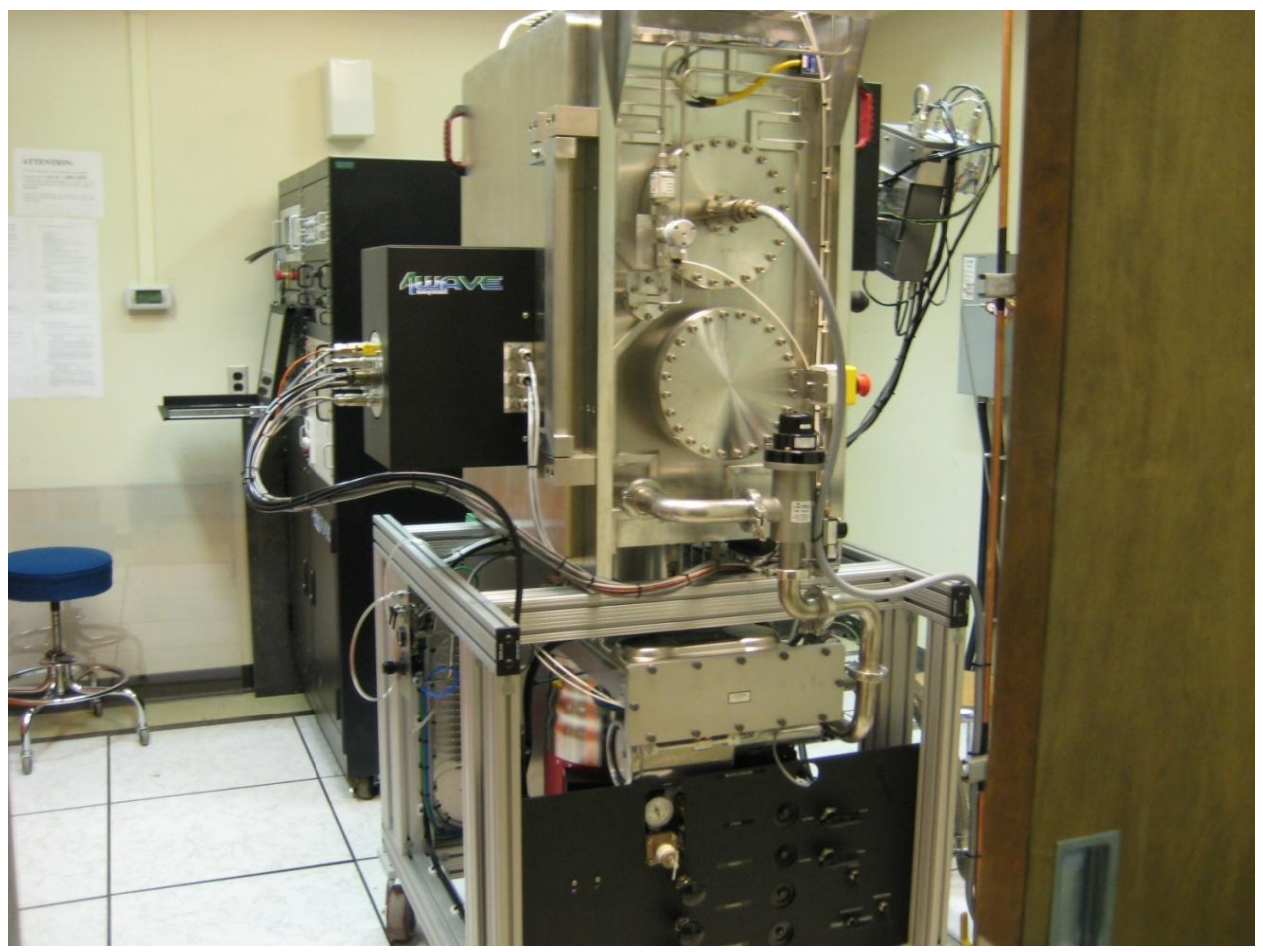

Figure 3.1 Picture of the BTIBD system 


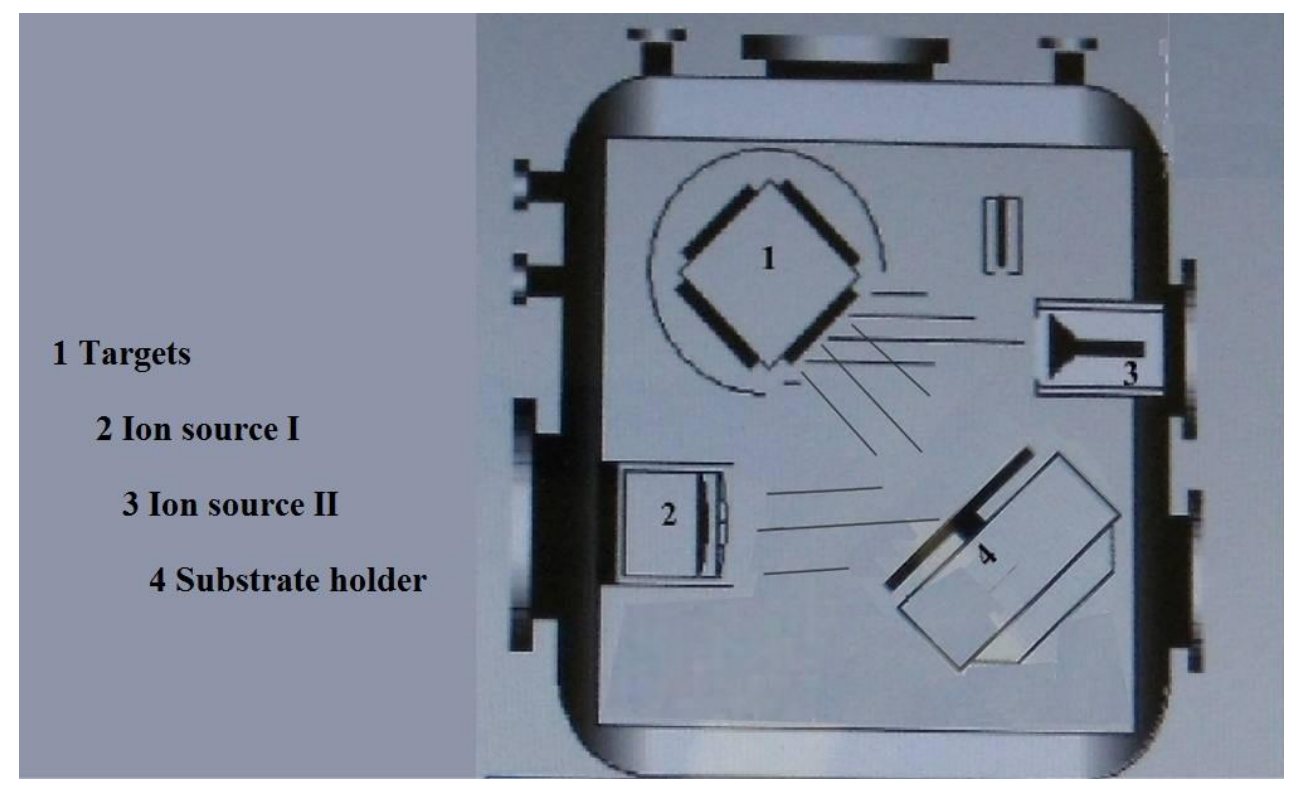

Figure 3.2 Schematic of the BTIBD system

Figure 3.2 presents a schematic diagram of the configuration of the system. As shown in the figure, there are a target assembly, two ion sources, a substrate stage, shutters, and targets in the vacuum chamber. The substrate stage is rotatable, and can be cooled or heated. It can also be inclined from $0^{\circ}$ to $90^{\circ}$. Ion source I is for direct ion beam deposition, or assisting deposition, or precleaning, or etching, while ion source II is for sputtering. This system is versatile and can be used for ion beam deposition, biased target ion beam sputtering as well as ion beam etching. DLC thin films can be deposited either by direct ion beam deposition or by biased target ion beam sputtering deposition. Direct ion beam deposition uses a hydrocarbon gas as carbon source to produce hydrogenated DLC thin films, whereas ion beam sputtering deposition uses sputtered graphite as carbon source to produce hydrogen free DLC thin films. Nanocomposite DLC thin films can also be deposited using the system by applying ion beam deposition and biased target ion beam sputtering simultaneously. Metal or non-metal atoms/ions/clusters are generated by the biased target ion beam sputtering and incorporated into the DLC matrix which is simultaneously 
generated by the ion beam deposition. It's possible to realize the control of the content of metal or non-metal in the DLC films using the BTIBD system.

In the present thesis work, the ion sources used for the experiments are KRI EH-1000, manufactured by Kaufman \& Robinson, Inc., USA. EH-1000 is a gridless ion source which can produce a high ion current (up to $5 \mathrm{Amps})$ and low energy ion beam (35 - $210 \mathrm{eV})$ and uniformly cover large substrate areas. It is ideally suited for thin film growth application with the advantage of easy operation and maintenance. B-DLC thin films were synthesized by simultaneously running the two EH-1000 ion sources, one for deposition of hydrogenated DLC from methane and the other for B doping by sputtering $\mathrm{B}_{4} \mathrm{C}$ target. Pure hydrogenated DLC films and $\mathrm{B}-\mathrm{C}$ thin films were also prepared by direct ion beam deposition and ion beam sputtering of $\mathrm{B}_{4} \mathrm{C}$ target, respectively, as reference samples and for the control of film thickness and B doping concentration in B-DLC films.

For all the experiments of thin film deposition, p-type (100) mirror-polished $10 \mathrm{~cm}$ (diameter) silicon wafers were used as substrates. Prior to each thin film deposition, the substrate was chemically cleaned in an ultrasonic bath for 30 minutes with 95\% ethyl alcohol and then placed into the vacuum chamber on the sample stage for deposition. The sample stage was located 300 $\mathrm{mm}$ away from the ion source. It tilted $45^{\circ}$ with respect to the ion source and meantime rotated around its axis at a speed of $2 \mathrm{rpm}$ for better film uniformity. In the vacuum chamber, the background pressure was about $1.1 \times 10^{-7}$ Torr. Each film deposition was performed around a pressure of $7.6 \times 10^{-4}$ Torr without any extra heating or cooling. The substrate holder temperature was close to room temperature. More detailed experimental procedures and parameters will be given in the following sections. 


\subsection{B-C Thin Film Deposition by Ion Beam Sputtering of $\mathrm{B}_{4} \mathrm{C}$ Target}

B-C thin films were prepared using ion beam sputtering under different conditions to estimate the sputtering rate for the control of B contents in B-DLC films. In these experiments, only the sputter $\mathrm{EH}$ ion source was in operation in the BTIBD system. A $\mathrm{B}_{4} \mathrm{C}$ target of $99 \%$ purity was sputtered by the sputter ion source using Ar gas in the vacuum chamber for film deposition on the cleaned silicon wafers.

Different processing parameters such as target bias voltage, ion source anode current, deposition time, as shown in Table 3.1, with a fixed Ar gas flow of $50 \mathrm{sccm}$, were applied to prepare B-C thin films. Both the target bias voltage and ion source anode current have effects on sputtering rate, and thus film deposition rate. Therefore the target bias voltages changing from 100 to $800 \mathrm{~V}$ (900 V maximum) at a medium ion source anode current and the ion source anode currents changing from 1 to $4 \mathrm{~A}$ ( 5 A maximum) at a medium target bias voltage were used to investigate the effects of these parameters on the deposition rate. The mass of silicon wafers before $\left(\mathrm{W}_{0}\right)$ and after $\left(\mathrm{W}_{\mathrm{i}}\right)$ film deposition was measured and recorded, respectively, as shown in Table 3.1. The weight increase $\left(\mathrm{W}_{\mathrm{i}}-\mathrm{W}_{0}\right)$ due to the deposition was used to estimate the film thickness (T). The deposition duration was set long enough to make sure the weight increase was measurable. Assuming the film density and silicon wafer area are $\rho$ and a, respectively, we can estimate the film thickness using $\mathrm{T}=\left(\mathrm{W}_{\mathrm{i}}-\mathrm{W}_{0}\right) / \rho$ a. Then, the deposition rate $(\mathrm{R})$ can be estimated by $\mathrm{T}$ divided by deposition duration. 
Table 3.1 Sputtering parameters and estimated deposition rates on silicon

\begin{tabular}{|c|c|c|c|c|c|c|}
\hline Parameters/Records & $\mathrm{B}_{4} \mathrm{C}-1$ & $\mathrm{~B}_{4} \mathrm{C}-2$ & $\mathrm{~B}_{4} \mathrm{C}-3$ & $\mathrm{~B}_{4} \mathrm{C}-4$ & $\mathrm{~B}_{4} \mathrm{C}-5$ & $\mathrm{~B}_{4} \mathrm{C}-6$ \\
\hline Target bias voltage (V) & 800 & 100 & 400 & 400 & 400 & 200 \\
\hline Anode current (A) & 4 & 4 & 4 & 2 & 1 & 4 \\
\hline $\begin{array}{l}\text { Wafer mass before } \\
\text { film deposition }(\mathrm{g})\end{array}$ & 9.30805 & 9.85130 & 9.84280 & 9.92090 & 9.72430 & 9.72550 \\
\hline $\begin{array}{l}\text { Wafer mass after } \\
\text { film deposition (g) }\end{array}$ & 9.32010 & 9.85195 & 9.84510 & 9.92245 & 9.72535 & 9.72630 \\
\hline Deposition duration (h) & 48 & 48 & 24 & 24 & 24 & 13 \\
\hline Film thickness $(\mu \mathrm{m})$ & 0.61 & 0.03 & 0.12 & 0.08 & 0.05 & 0.04 \\
\hline Deposition rate $(\mathrm{nm} / \mathrm{h})$ & 13 & 1 & 5 & 3 & 2 & 3 \\
\hline
\end{tabular}

In those experiments, the area of silicon wafers is $\pi \cdot(5 \mathrm{~cm})^{2}=78.5 \mathrm{~cm}^{2}$, and the density of $\mathrm{B}_{4} \mathrm{C}, \rho=2.5 \mathrm{~g} / \mathrm{cm}^{3}$, is used for the calculation. The estimated film thickness and deposition rate are also shown in Table 3.1. It can be seen that the increase of target bias voltage and ion source anode current leads to the increase of deposition rate.

\subsection{Deposition of DLC and B-DLC Thin Films}

Pure hydrogenated DLC thin films were deposited by direct ion beam deposition. In these experiments, only the deposition ion source was in operation in the BTIBD system. Precursor gases $\mathrm{CH}_{4}$ and Ar were introduced into the ion source to produce carbon/hydrocarbon ions and the ions were directed toward the cleaned silicon substrates for deposition of thin films.

Different processing parameters such as $\mathrm{Ar}$ and $\mathrm{CH}_{4}$ flow rates, ion source anode current were applied with a maximum ion source anode voltage setting for DLC film deposition, as shown in 
Table 3.2. The applied parameters were chosen based on previous successful DLC deposition experiments. The mean anode voltage listed in Table 3.2 is a calculated value. With a maximum ion source anode voltage set, there is actually fluctuating anode voltage observed during experiments even though the anode current and gas flow rates keep constant. The mean anode voltage is reported typically as $65 \%$ of the actual anode voltage range for every experiment. The observed actual anode voltage range representing ion energy is closely related to the Ar flow rate. Higher Ar flow rate gets the actual anode voltage range down by increasing electrical resistance. The anode current determines rates of ions arriving onto the substrate surface and high current may etch off synthesized thin films. The mass of silicon wafers before and after film deposition was measured and recorded, respectively, as shown in Table 3.2.

Table 3.2 DLC deposition parameters on silicon

\begin{tabular}{|c|c|c|c|c|}
\hline Parameters/Records & DLC-1 & DLC-2 & DLC-3 & DLC-4 \\
\hline Maximum anode voltage setting (V) & 150 & 150 & 150 & 150 \\
\hline Mean anode voltage $(\mathrm{V})$ & 134 & 134 & 121 & 121 \\
\hline Anode current $(\mathrm{A})$ & 4 & 2.5 & 2.5 & 2.5 \\
\hline Ar flow rate $(\mathrm{sccm})$ & 8 & 8 & 10 & 10 \\
\hline $\mathrm{CH}_{4}$ flow rate $(\mathrm{sccm})$ & 6 & 6 & 6 & 6 \\
\hline $\begin{array}{l}\text { Wafer mass before } \\
\text { film deposition (g) }\end{array}$ & 9.30905 & 9.85115 & 9.30870 & 10.07500 \\
\hline $\begin{array}{l}\text { Wafer masst after } \\
\text { film deposition (g) }\end{array}$ & 9.30105 & 9.84800 & 9.30365 & 10.07450 \\
\hline Deposition duration $(\mathrm{h})$ & 2 & 2 & 5 & 10 \\
\hline Film thickness ( $\mu \mathrm{m})$ & 0 & 0 & 0.27 & 0.53 \\
\hline
\end{tabular}


In the experiments, there was no film synthesized on DLC-1 and DLC-2, which was observed after experiment and further testified by Raman spectroscopy. Upon reducing the anode current and lowering the anode voltage by increasing the Ar flow rate, films were successfully synthesized on DLC-3 and DLC-4. For all the four silicon wafers, the measured wafer mass after film deposition is less than the mass before film deposition, which may be due to the ion etching caused by the ion beam directing to the silicon wafer surface at the beginning of deposition experiments (before the DLC thin films form). Since DLC-3 and DLC-4 were deposited under the same processing parameters, the deposition rate $(\mathrm{R})$ is supposed to be the same and can be estimated using DLC-3 and DLC-4 relative wafer mass change $\left(\triangle W_{4}-\triangle W_{3}\right)$ and deposition duration difference $(\triangle D)$. Assuming the DLC film density and silicon wafer area are $\rho$ and a respectively, then $R=\left(\triangle W_{4}-\triangle W_{3}\right) /(\rho \mathrm{a} \triangle D)$. In those experiments, a is $\pi \cdot(5 \mathrm{~cm})^{2}=78.5 \mathrm{~cm}^{2}$, and $\rho$ is $2.2 \mathrm{~g} / \mathrm{cm}^{3}$, thus the deposition rate is calculated to be $53 \mathrm{~nm} / \mathrm{h}$. This estimated deposition rate is in a reasonable range. Since the processing parameters used for DLC-3 and DLC-4 worked well, they were used further for synthesis of B-DLC thin films.

For the deposition of B-DLC thin films, both the EH ion sources were in operation in the BTIBD system. Precursor gases $\mathrm{CH}_{4}$ and Ar were introduced into one $\mathrm{EH}$ ion source to produce hydrocarbon ions for DLC deposition on the silicon substrates. The $\mathrm{B}_{4} \mathrm{C}$ target of $99 \%$ purity was simultaneously sputtered by the Ar ions from the other ion source to produce B atoms/ions for deposition on the silicon substrates. The deposition duration for all the B-DLC film samples was 4 hours achieving film thickness of about $0.24 \mu \mathrm{m}$.

In the B-DLC film deposition experiments, the processing parameters for deposition ion source were the same for pure DLC deposition. In order to prepare samples with different B 
content, the processing parameters for sputtering of $\mathrm{B}_{4} \mathrm{C}$ target (target bias voltage and anode current) varied as shown in Table 3.3.

Table 3.3 Deposition parameters for B-DLC films on silicon

\begin{tabular}{lccccc}
\hline & B-DLC-1 & B-DLC-2 & B-DLC-3 & B-DLC-4 & B-DLC-5 \\
\hline \hline L.E.E.S maximum & anode & voltage & & & \\
setting (V) & 150 & 150 & 150 & 150 & 150 \\
L.E.E.S mean anode voltage (V) & 109 & 109 & 109 & 109 & 109 \\
L.E.E.S anode current (A) & 2.5 & 2.5 & 2.5 & 2.5 & 2.5 \\
Ar flow rate (sccm) & 10 & 10 & 10 & 10 & 10 \\
CH flow rate (sccm) & 6 & 6 & 6 & 6 & 6 \\
Target bias voltage (V) & 400 & 400 & 200 & 200 & 200 \\
Anode current (A) & 4 & 2 & 4 & 2 & 1 \\
Deposition duration (h) & 4 & 4 & 4 & 4 & 4 \\
Estimated B wt. \% & 8 & 5 & 5 & 3 & 2 \\
\hline
\end{tabular}

In the above table, L.E.E.S represents low energy etch source which is the ion source for DLC deposition. Assuming the estimated deposition rate and the density of $\mathrm{B}_{4} \mathrm{C}$ sputtered film and pure DLC film are $R_{B 4 C}, R_{D L C}$ and $\rho_{B 4 C}, \rho_{D L C}$, respectively, then the $B$ weight percentage in the synthesized B-DLC film can be estimated by B wt. $\%=\left[R_{B 4 C} \cdot \rho_{\mathrm{B} 4 \mathrm{C}} /\left(\mathrm{R}_{\mathrm{B} 4 \mathrm{C}} \cdot \rho_{\mathrm{B} 4 \mathrm{C}}+\right.\right.$ $\left.\left.\mathrm{R}_{\mathrm{DLC}} \cdot \rho_{\mathrm{DLC}}\right)\right] \cdot[4 \cdot 10.81 /(4 \cdot 10.81+12.01)]$, where 10.81 is $\mathrm{B}$ atomic weight and 12.01 is $\mathrm{C}$ atomic weight. In those experiments, $\rho_{\mathrm{B} 4 \mathrm{C}}=2.5 \mathrm{~g} / \mathrm{cm}^{3}, \rho_{\mathrm{DLC}}=2.2 \mathrm{~g} / \mathrm{cm}^{3}, \mathrm{R}_{\mathrm{DLC}}=53 \mathrm{~nm} / \mathrm{h}$ and different corresponding $\mathrm{R}_{\mathrm{B} 4 \mathrm{C}}$ were used for calculation. The estimated $\mathrm{B}$ wt. \% is also shown in 
Table 3.3. The B weight percentage in the B-DLC films increases with the increase of target bias voltage and ion current density applied for sputtering of $\mathrm{B}_{4} \mathrm{C}$ target, as shown in Table 3.3.

\subsection{Characterization of Thin Films}

\subsubsection{Chemical and Structural Characterization}

A JEOL JSM-6010LV SEM (Jeol Ltd., Tokyo, Japan) located in the Department of Mechanical Engineering, University of Saskatchewan, was used to observe the surface morphology and cross-section of the synthesized film samples. A picture of the SEM instrument is shown in Figure 3.3. The SEM was operated at an accelerating voltage of $15 \mathrm{kV}$ for all the samples. A PicoSPM AFM (Molecular Imaging, Tempe, AZ) at the Saskatchewan Structural Science Centre (SSSC), University of Saskatchewan, was used to obtain high resolution images

of the surface topography of the synthesized films and to measure their surface roughness. Figure 3.4 presents a picture of the AFM instrument. The AFM was operated in intermittent contact mode applying a silicon cantilever (AppNano FORT-SS, Nanoscience Instruments, Tempe, AZ) with a curvature radius of $<4 \mathrm{~nm}$, a force constant of approximately $3 \mathrm{~N} / \mathrm{m}$, and a resonant frequency of approximately $60 \mathrm{kHz}$. All the AFM measurements were conducted at a set-point ratio of approximately $0.8-0.9$ from the free-amplitude of the cantilever and they were all done in a vibration isolation system with the scan rate of $0.5-1.0 \mathrm{~Hz}(512$ pixels per line) for all the images. 


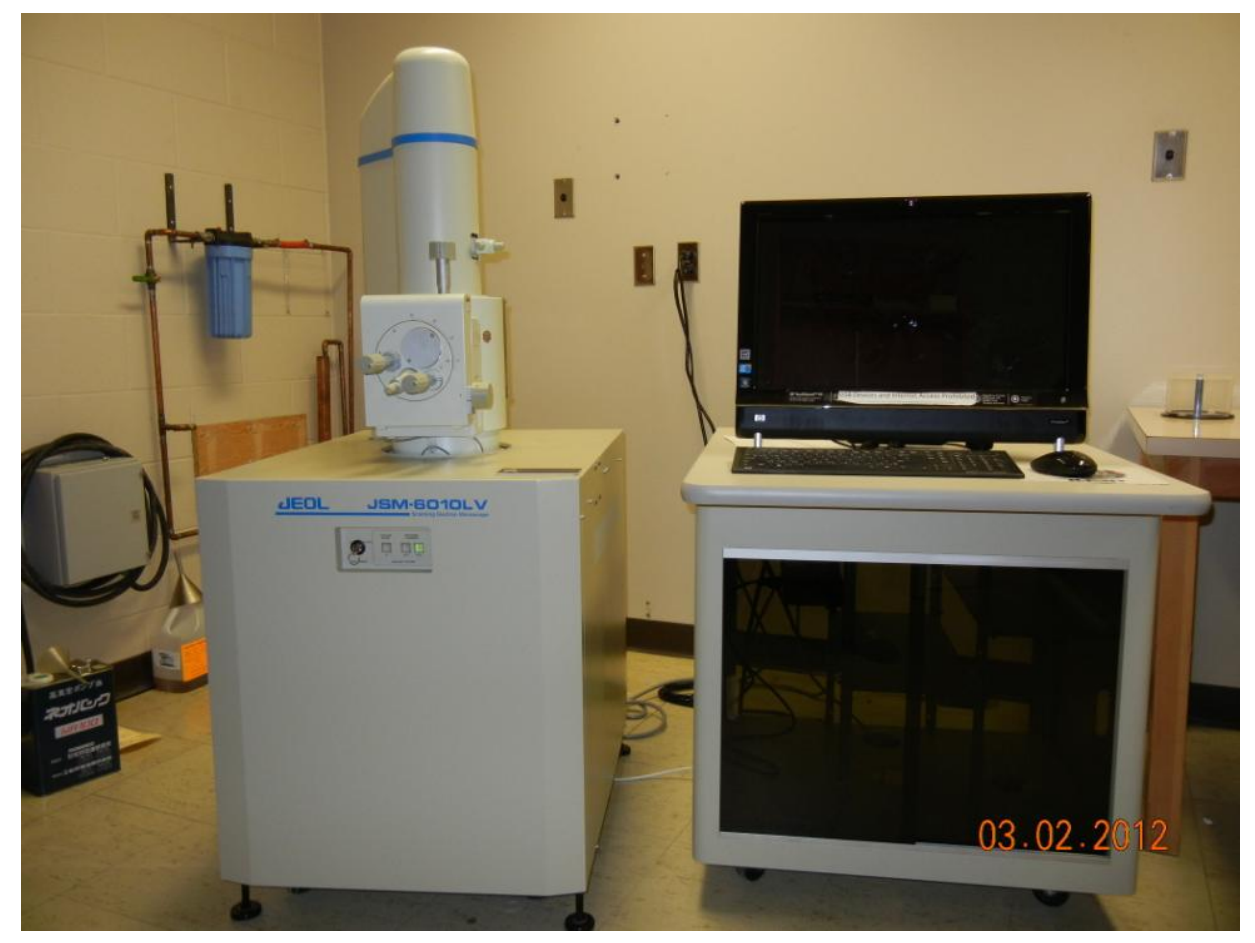

Figure 3.3 Scanning electron microscopy - JEOL JSM-6010LV

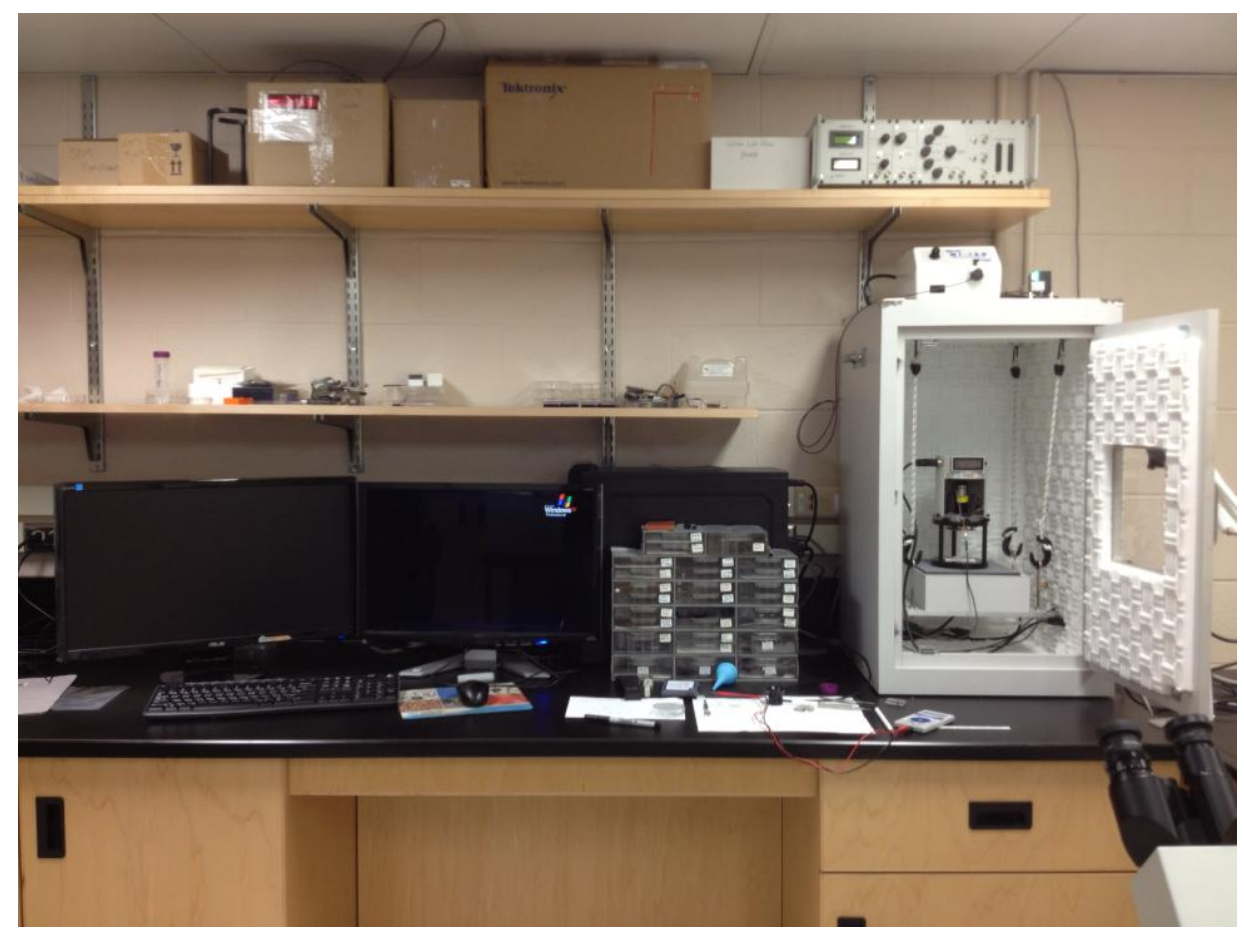

Figure 3.4 Atomic force microscopy - PicoSPM 
A Rigaku (Rotaflex Ru-200) X-ray diffractometer located in the Plasma Physics Laboratory, University of Saskatchewan, was used to characterize the crystal structure of the samples. A picture of the X-ray diffractometer is given in Figure 3.5. Co $\mathrm{K} \alpha$ line $(\lambda=0.178897 \mathrm{~nm})$ produced by impinging a $30 \mathrm{kV}, 20 \mathrm{~mA}$ electron beam on a Co target was used. The sample and the detector are mounted separately on two different rotating bases. When the sample rotates an angle $\theta$, the detector rotates an angle $2 \theta$. The thin film samples were scanned slowly $\left(0.01^{\circ} / 2 \mathrm{sec}\right)$ from $20^{\circ}$ to $55^{\circ}$ for $2 \theta$.

Raman spectra of the synthesized films were obtained using a Renishaw 2000 micro-Raman system located at the SSSC, University of Saskatchewan. This Raman spectroscope was operated using an Ar ion laser with a wavelength of $514.5 \mathrm{~nm}$ and a spot size of approximately $2 \mu \mathrm{m}$. Figure 3.6 shows a picture of the Raman spectroscope.

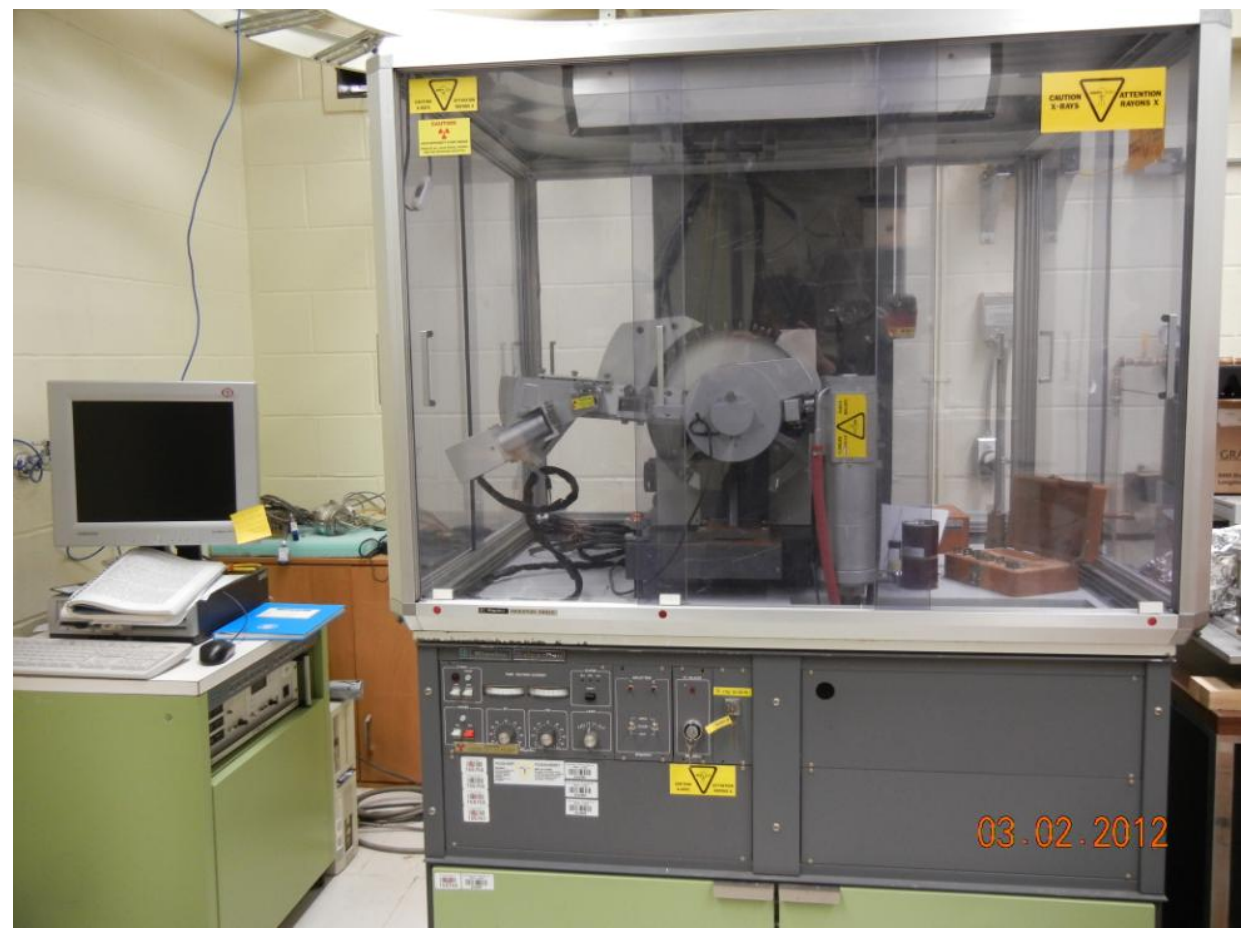

Figure 3.5 X-ray diffractometer - Rigaku 


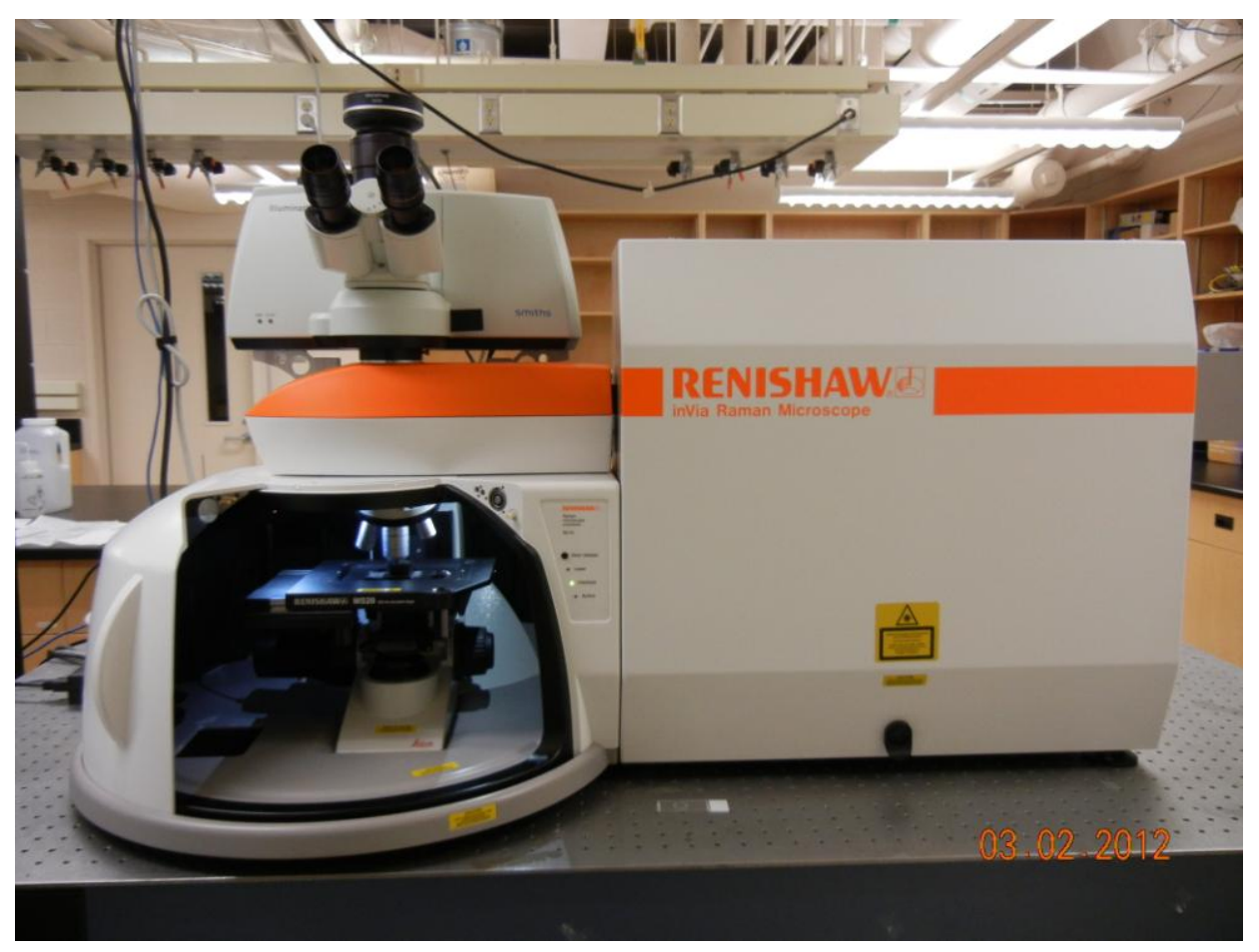

Figure 3.6 Raman spectroscope - Renishaw model 2000

XPS measurements were performed at the Canadian Light Source Inc. (CLS), University of Saskatchewan, using the Spherical Grating Monochromator (SGM) beam line to study the chemical composition and elemental bonding states of the synthesized films. The photon energy was set to $1000 \mathrm{eV}$ for the measurements. The instrumental resolution of the XPS data is less than $0.1 \mathrm{eV}$. The CLS scientists, Dr. Xiaoyu Cui and Dr. Yongfeng Hu, conducted the XPS measurements and reported the data. NEXAFS measurements were also performed using the SGM beam line at the CLS, University of Saskatchewan. Both the surface sensitive TEY spectra and bulk sensitive FLY spectra were collected and reported with the help of the CLS scientists, Dr. Qunfeng Xiao and Dr. Yongfeng Hu. The photon resolution of the NEXAFS measurements is less than $0.1 \mathrm{eV}$. 


\subsubsection{Mechanical and Friction Characterization}

A Universal Mechanical Tester (UMT) manufactured by Center for Tribology Inc. and located at the Department of Mechanical Engineering, University of Saskatchewan, was used to conduct nanoindentation testing to measure the hardness and elastic modulus of the films synthesized on Si substrates. Figure 3.7 shows a picture of the UMT. The $100 \mathrm{~nm}$ Berkovich indenter tip used for the nanoindentation testing was normal to the film surfaces and driven into the films by applying an increasing load up to a preset value of $0.5 \mathrm{mN}$. After 10 second holding, the load was gradually decreased until the films were relaxed. The loading and unloading speed was $0.5 \mathrm{mN} / \mathrm{min}$. The load and displacement were recorded continuously throughout the whole process and used to calculate the film hardness and Young's modulus by the inbuilt software based on the Oliver and Pharr method (Oliver et al., 2004). Multiple indents (9) on a specific film area were obtained automatically with a lateral and longitudinal resolution of $50 \mu \mathrm{m}$.

The same UMT was also used to perform ball-on-disk friction testing to investigate the friction behavior of the synthesized thin films. The friction tests were conducted in a reciprocating mode using a $4 \mathrm{~mm}$ diameter stainless steel ball as the counterpart material. The sliding distance is $10 \mathrm{~mm}$ and the sliding speed is $1 \mathrm{~mm} / \mathrm{s}$. The normal force applied was kept at 2 N. Friction force $\left(\mathrm{F}_{\mathrm{x}}\right)$, normal load $\left(\mathrm{F}_{\mathrm{z}}\right)$, and coefficient of friction $(\mathrm{COF})$ were measured, recorded and calculated. 


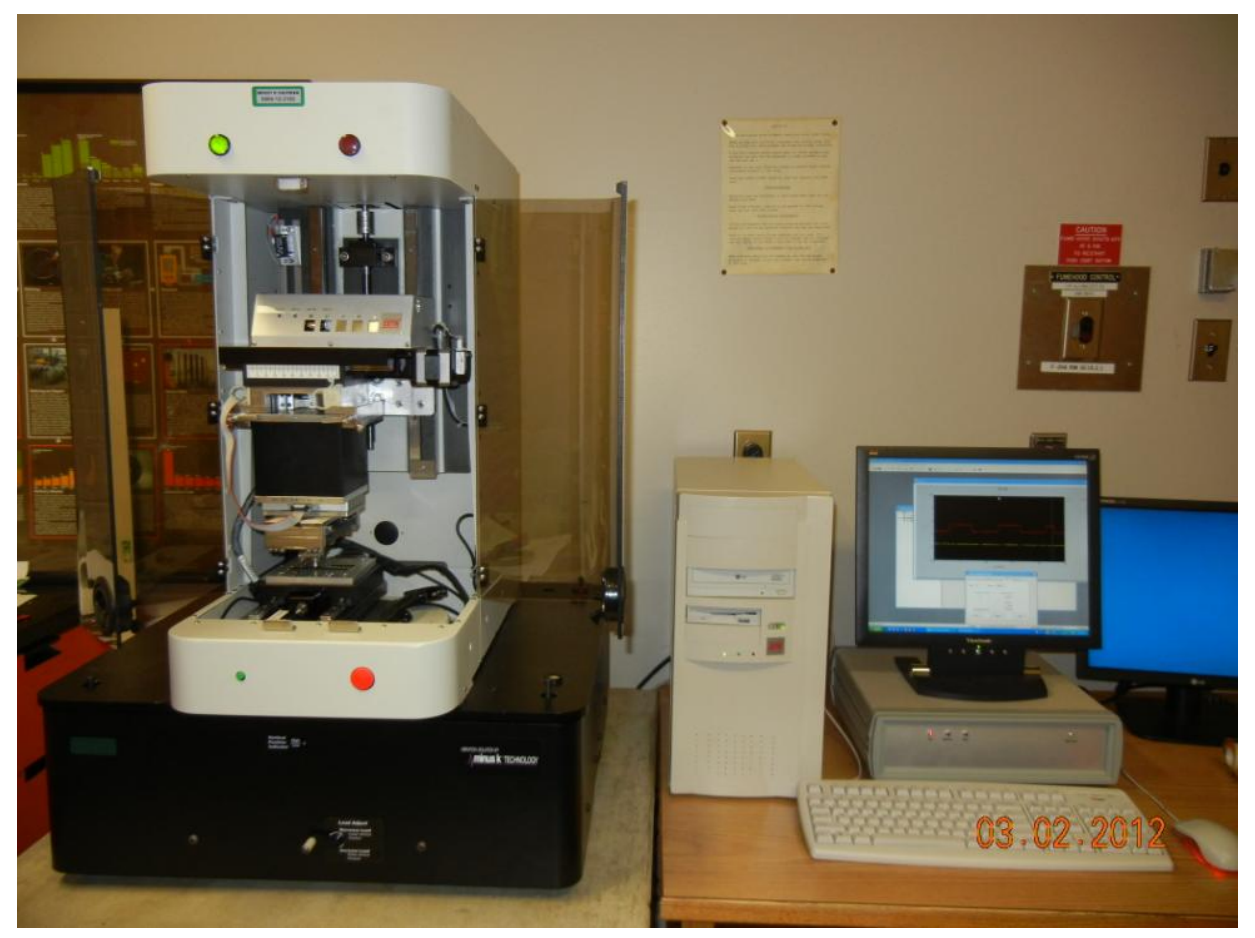

Figure 3.7 Universal Mechanical Tester 


\section{CHAPTER 4 \\ RESULTS AND DISCUSSION}

\subsection{SEM Observation}

Both the surface and cross-section of film samples were observed using SEM. The cross-section images of B-C film samples by $\mathrm{B}_{4} \mathrm{C}$ sputtering are shown in Figure 4.1 and 4.2. The films are dense and uniform with sharp interface to the substrates. They are also continuous and very smooth over the whole substrates, without any special features. The average film thickness obtained from the SEM observation is $0.73 \mu \mathrm{m}$ for B-C-1 and $0.16 \mu \mathrm{m}$ for B-C-3, respectively, which is slightly higher than that estimated by mass changes in Section 3.2. The film thickness of B-C-1 and B-C-4 was also measured by a profilometer and found to be averagely $654 \mathrm{~nm}$ and $96 \mathrm{~nm}$ respectively. These values are in higher accuracy comparing to those obtained from the SEM observation and they are very close to the calculated film thickness $0.61 \mu \mathrm{m}$ for B-C-1 and $0.08 \mu \mathrm{m}$ for B-C-4. This further proves that the calculation method to estimate the film thickness in Section 3.2 is workable, which brings acceptable estimation/ calculation for the film deposition rate.

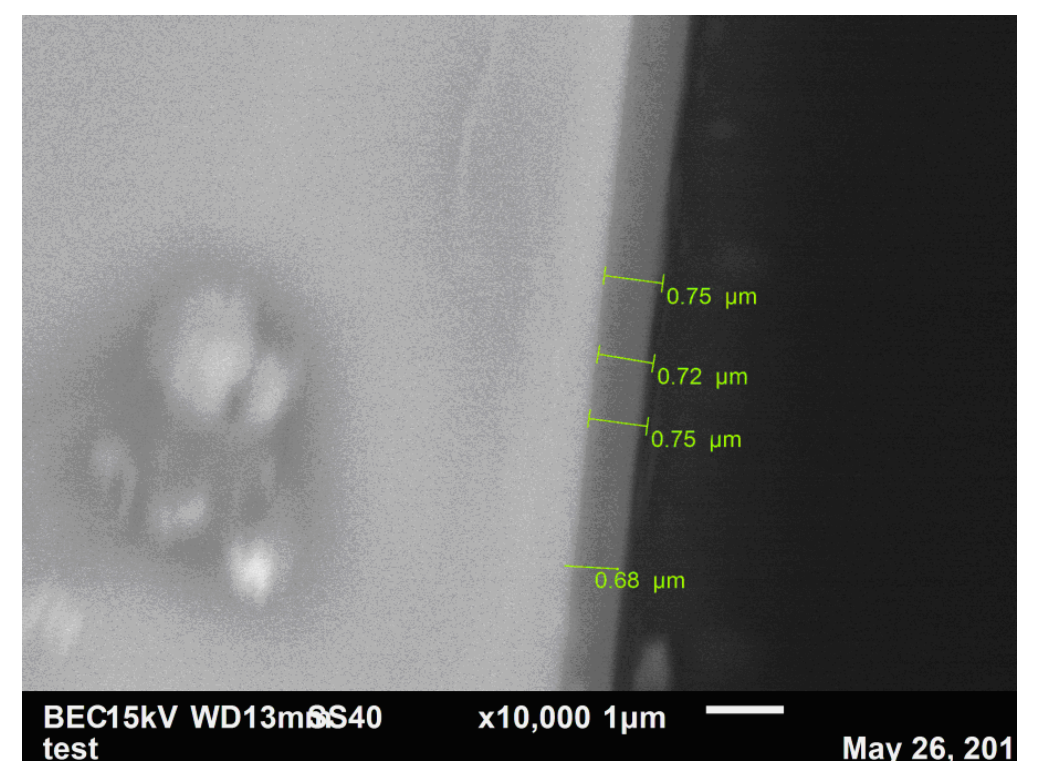

Figure 4.1 Cross-section SEM image of B-C-1 (calculated film thickness $0.61 \mu \mathrm{m}$ ) 


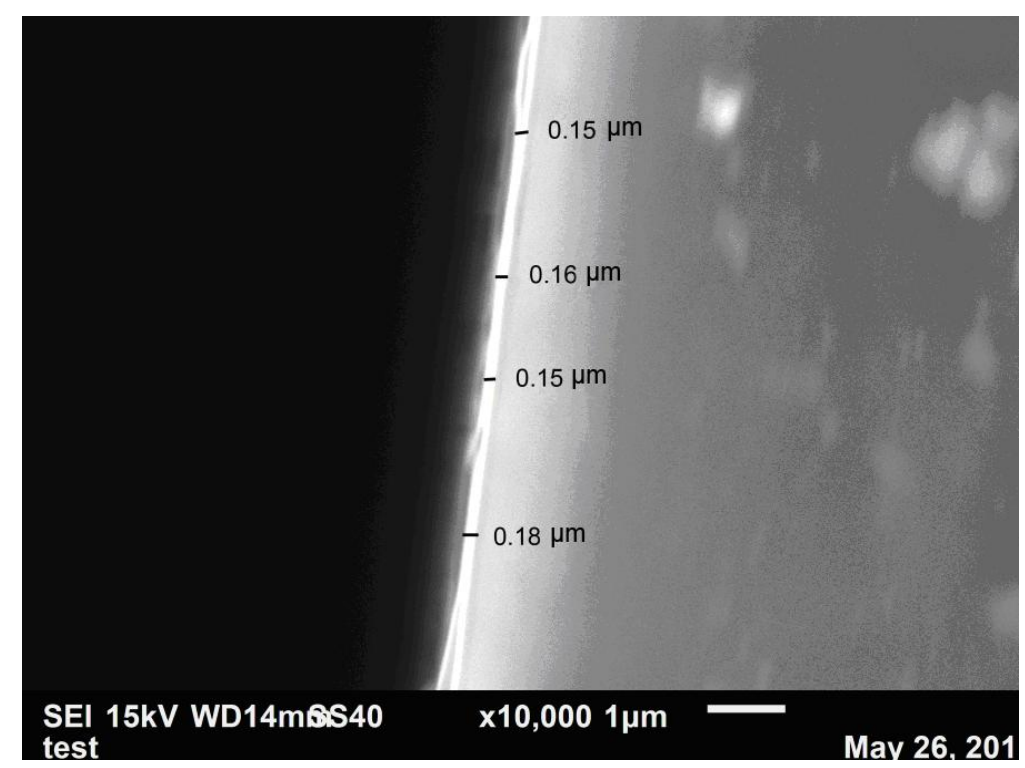

Figure 4.2 Cross-section SEM image of B-C-3 (calculated film thickness $0.12 \mu \mathrm{m}$ )

The SEM images of the surfaces of B-C film, DLC and B-DLC films were obtained, as shown in Figure 4.3. All the images are similar and reveal that the films are dense, smooth and uniform without pores and discontinuity. No special features such as grains were observed for all the films, which is consistent with the cross-section observation, indicating that the films might be of amorphous structure.

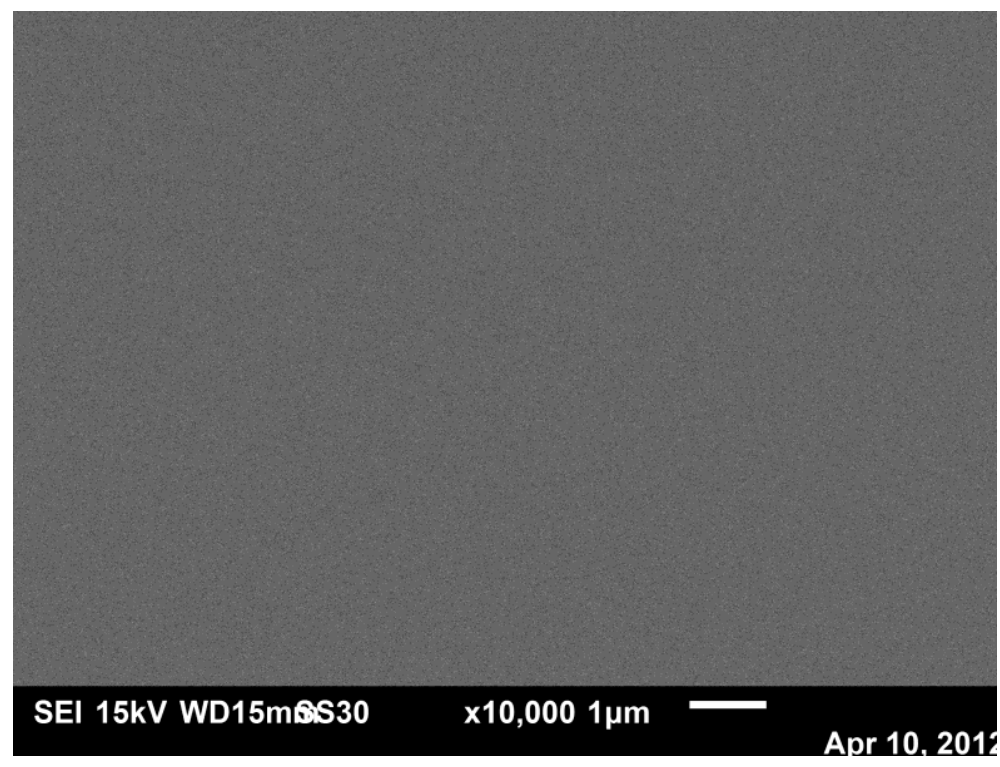

Figure 4.3 SEM image of B-DLC film 


\subsection{X-ray Diffraction}

X-ray diffraction was conducted on the B-C thin films prepared under different ion beam sputtering conditions with substrate temperature close to room temperature. The XRD pattern, as presented in Figure 4.4 ( $\theta$ is incident angle), shows no diffraction peaks, further indicating that the B-C films are likely of amorphous structure. There was no broad band of amorphous structure observed in the XRD patterns, which is probably due to the films being too thin. This investigation is consistent with what Chen et al. reported, where X-ray diffraction performed on the B-C films synthesized by ion beam sputtering of a bulk $\mathrm{B}_{4} \mathrm{C}$ target showed no diffraction peaks for the films with the substrate temperature under $100{ }^{\circ} \mathrm{C}$ (Chen et al., 2000). When the $\mathrm{B}_{4} \mathrm{C}$ target was sputtered by high energy Ar ions, the particles ejected from the target for the deposition of B-C films were mainly B and C atoms. Because of the low mobility of the deposition atoms at low substrate temperature, e.g. lower than $100{ }^{\circ} \mathrm{C}$, highly ordered B-C crystalline structure was difficult to form. Similarly, no XRD peaks were observed for DLC and B-DLC films, indicating that they are also likely of amorphous structure.

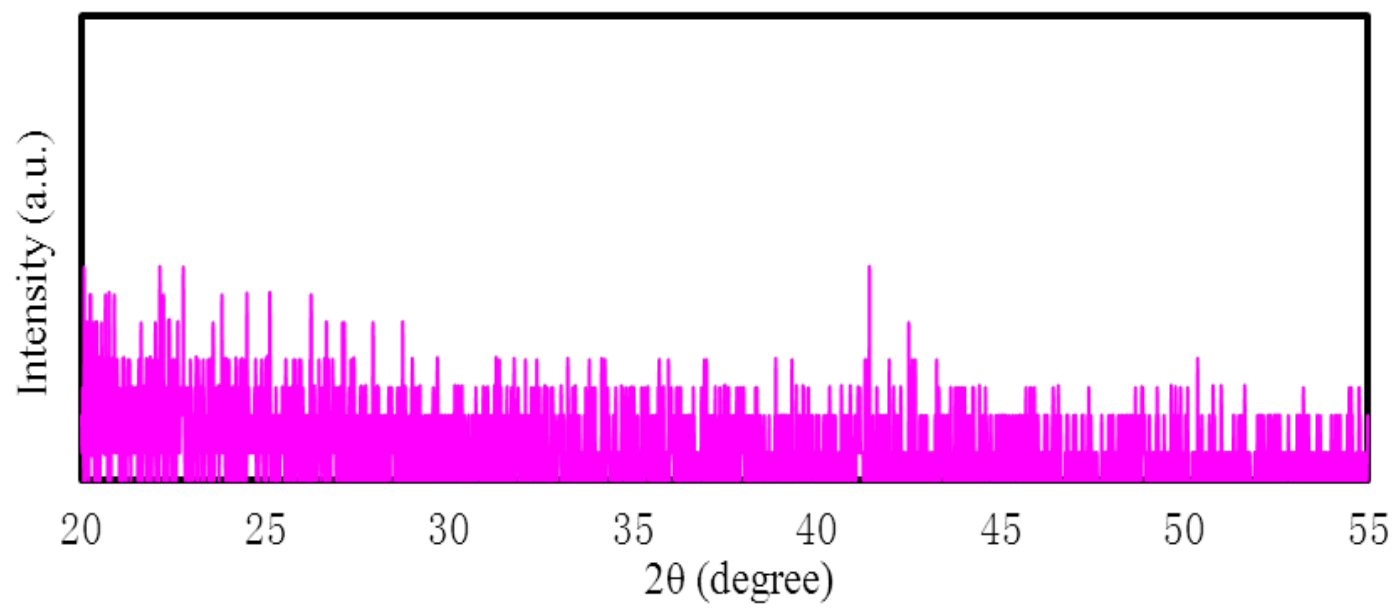

Figure 4.4 Typical XRD pattern of B-C thin film samples by $\mathrm{B}_{4} \mathrm{C}$ sputtering 


\subsection{Raman Spectra}

Figure 4.5 shows the Raman spectra of DLC thin films deposited on Si substrate with different B doping levels including pure DLC thin film. All spectra have a wide band ranging from 1100 to $1700 \mathrm{~cm}^{-1}$ that is featured with a G peak around $1580 \mathrm{~cm}^{-1}$ and a D peak around $1350 \mathrm{~cm}^{-1}$. Both D and G peaks are very broad, which is typical for amorphous carbon structures. With the increase of B doping level from DLC (without B doping) to B-DLC film 1 (highest B concentration), the relative intensities of $\mathrm{D}$ and $\mathrm{G}$ peaks do not change significantly. This suggests that a small amount of B doping does not significantly change the vibrational modes of carbon.

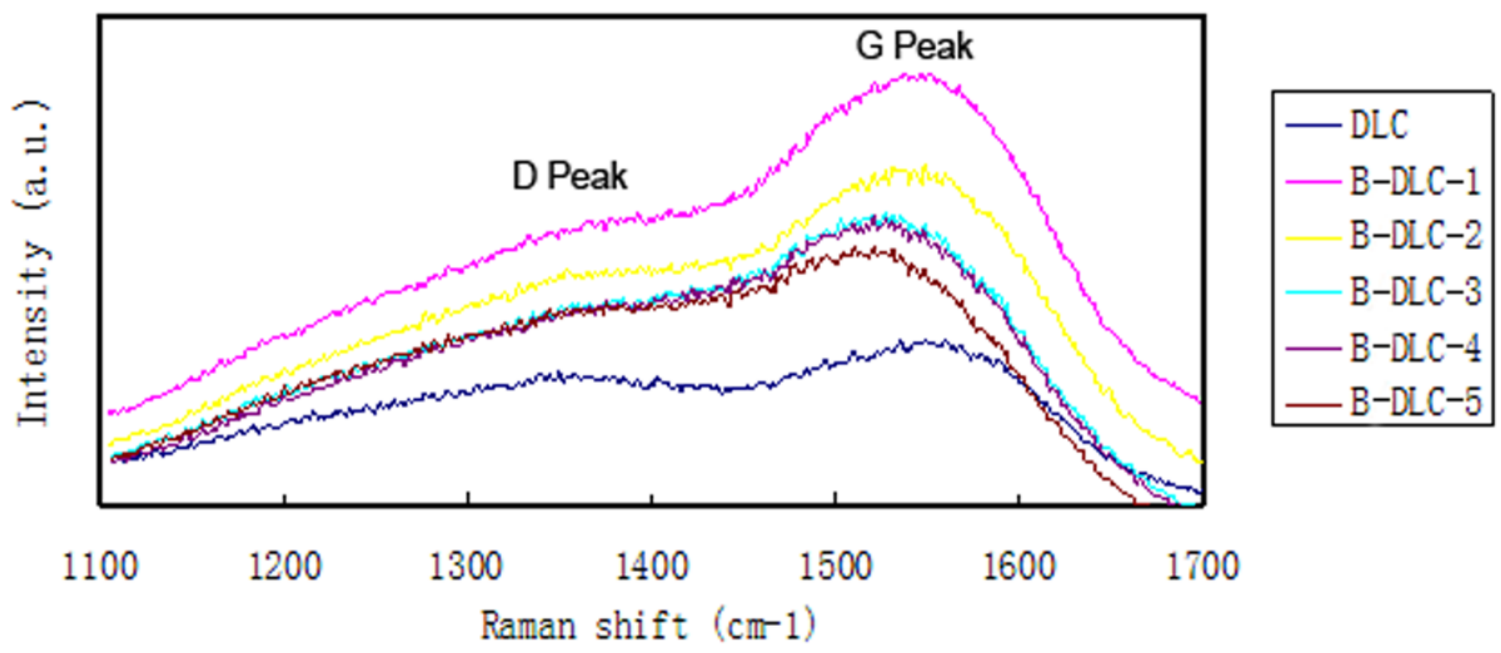

Figure 4.5 Raman spectra of pure DLC and B-DLC films

Previous research (Robertson, 2002) has reported that the ratio of integral area under D and G bands, $\mathrm{I}_{\mathrm{d}} / \mathrm{I}_{\mathrm{g}}$, is closely related to the ratio $\mathrm{sp}^{3} / \mathrm{sp}^{2}$ in DLC films, which is a very important factor to determine the properties of DLC based thin films. The Raman spectrum can be deconvoluted into a $\mathrm{G}$ band and a D band by a Gaussian line simulation where the fitted solid line agrees well with the raw experimental data. Figure 4.6, 4.7 and 4.8 show the Raman spectra of DLC film, B-DLC film 1 and 5 respectively, which were Gaussian-fitted with $G$ and D bands. The $\mathrm{I}_{d} / \mathrm{I}_{\mathrm{g}}$ 
values obtained from the Gaussian line simulations for all the thin film samples are listed in Table 4.1.

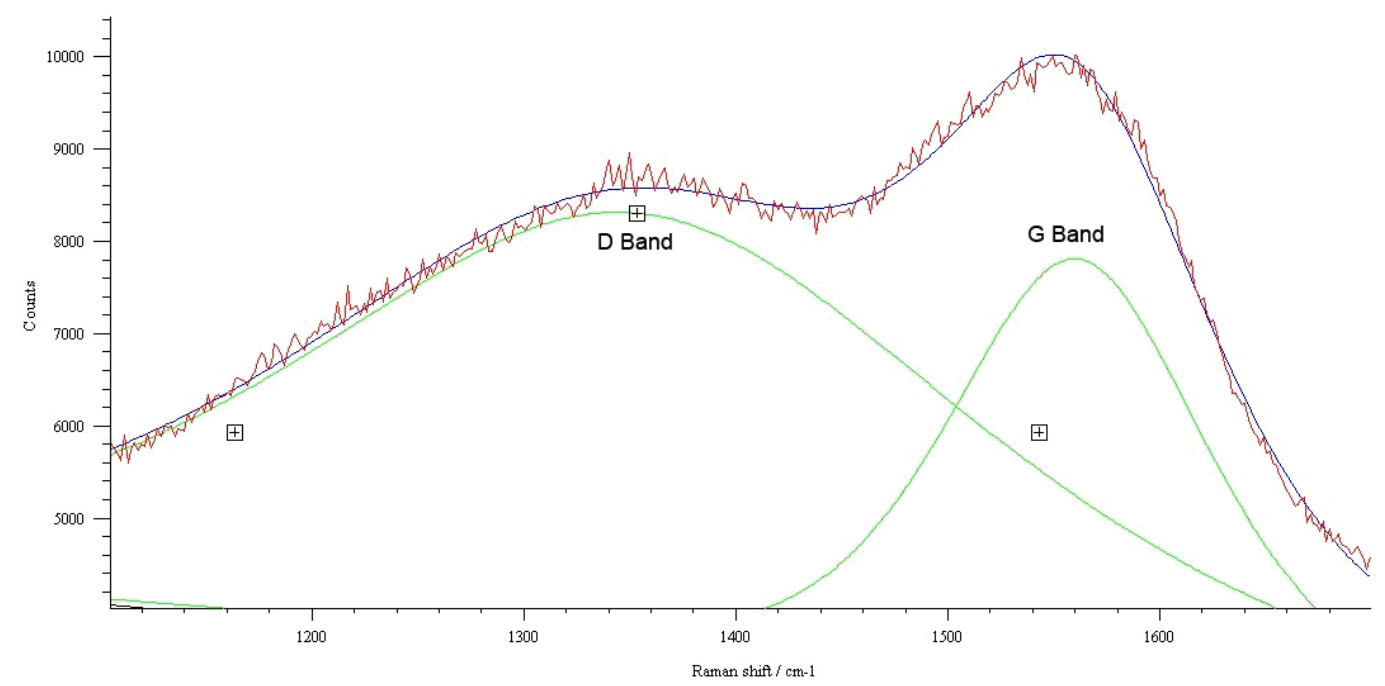

Figure 4.6 Gaussian-fitted Raman spectrum of DLC film $\left(\mathrm{I}_{\mathrm{d}} / \mathrm{I}_{\mathrm{g}}=1.01\right)$

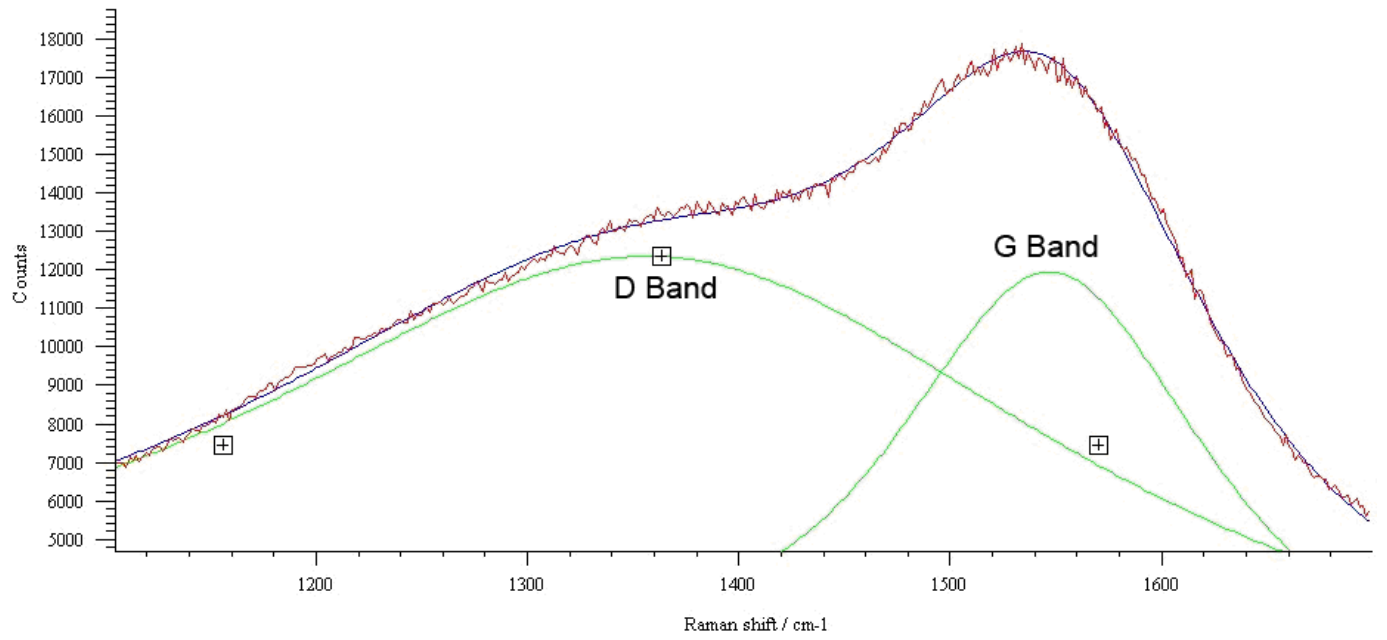

Figure 4.7 Gaussian-fitted Raman spectrum of B-DLC-1 $\left(\mathrm{I}_{\mathrm{d}} / \mathrm{I}_{\mathrm{g}}=0.99\right)$ 


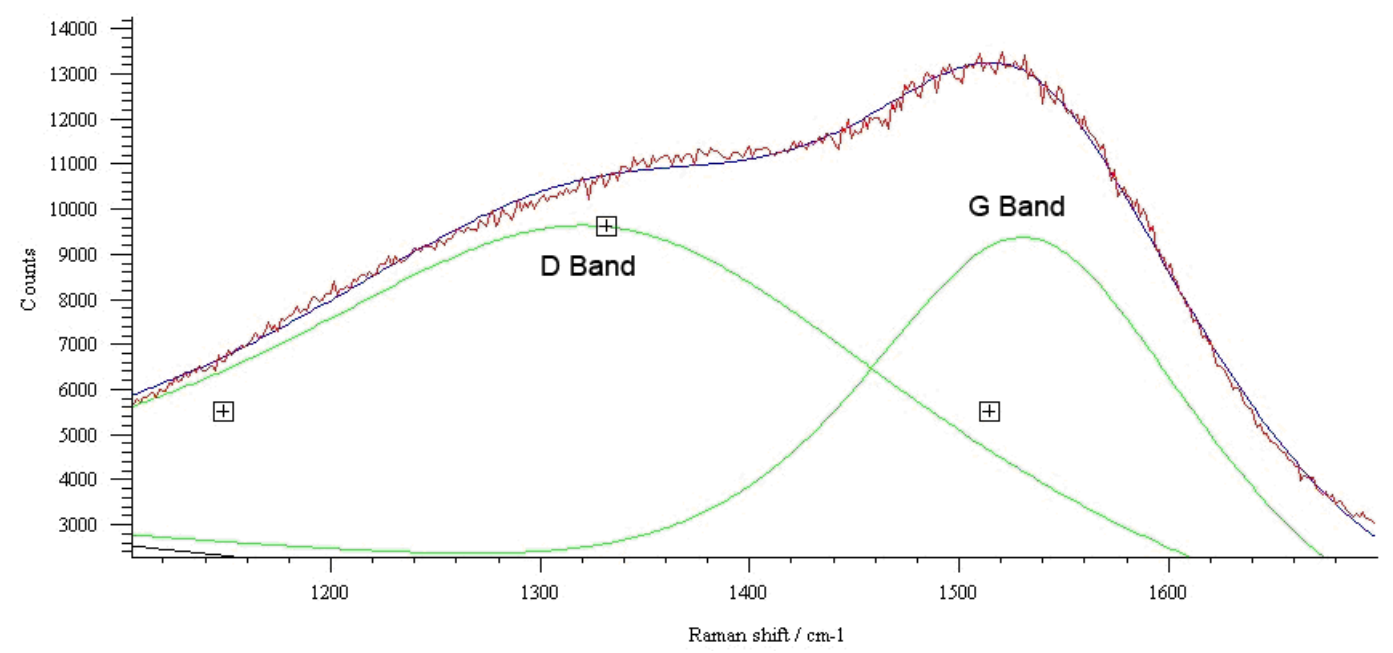

Figure 4.8 Gaussian-fitted Raman spectrum of B-DLC-5 $\left(\mathrm{I}_{\mathrm{d}} / \mathrm{I}_{\mathrm{g}}=0.92\right)$

Table 4.1 $\mathrm{I}_{\mathrm{d}} / \mathrm{I}_{\mathrm{g}}$ ratios for DLC and B-DLC thin films

\begin{tabular}{lcccccc}
\hline Samples & B-DLC-5 & B-DLC-4 & B-DLC-3 & B-DLC-2 & B-DLC-1 & DLC \\
\hline $\mathrm{I}_{\mathrm{d}} / \mathrm{I}_{\mathrm{g}}$ & 0.92 & 0.94 & 0.95 & 0.97 & 0.99 & 1.01 \\
\hline
\end{tabular}

As seen from Table 4.1, the DLC film has higher $I_{d} / I_{g}$ ratio than all the B-DLC films, and for the B-DLC films, the $\mathrm{I}_{d} / \mathrm{I}_{\mathrm{g}}$ ratio decreases with the decrease of B concentration in the films from B-DLC-1 (highest B concentration) to B-DLC-5 (lowest B concentration). According to the analysis of DLC Raman spectra reported previously (Ferrari et al., 2000), the $\mathrm{sp}^{3}$ bond content in DLC films increases with the decrease of $\mathrm{I}_{d} / \mathrm{I}_{\mathrm{g}}$ value. So the present Raman results suggest that the B doping into DLC films induces an increase of $\mathrm{sp}^{3}$ bonded carbon in the films and for the B-DLC films, the increase of B concentration (up to $8 \%$ ) seems to induce a decrease of $\mathrm{sp}^{3}$ bonded carbon in the films. This is also evident in Figure 4.5 from the shift of the G peak position (1561.3 $\mathrm{cm}^{-1}$ for the DLC film, B-DLC-5 film $1533.7 \mathrm{~cm}^{-1}$ to B-DLC-1 film 1548.6 $\left.\mathrm{cm}^{-1}\right)$. According to Tamor et al., the shift of G peak position from low Raman frequency to high Raman frequency corresponds to a decrease of $\mathrm{sp}^{3}$ carbon bond content (Tamor et al., 1994). Both the $G$ peak position and $I_{d} / I_{g}$ value of the deposited films show that the B doping into DLC 
films results in the increase of $\mathrm{sp}^{3}$ bonded carbon in the films, however, among the B-DLC films the increase of B concentration in the films seems to induce a decrease of $\mathrm{sp}^{3}$ bonded carbon.

The present results are consistent with those reported previously. He et al. reported that B incorporation to hydrogenated DLC up to 10 at. \% by radio frequency plasma-immersion ion deposition leads to an increase of $\mathrm{sp}^{3}$ carbon bonding in the films (He et al., 2000). Monteiro reported that B incorporation to hydrogen-free DLC in low concentrations by filtered cathodic arc deposition results in $\mathrm{sp}^{3}$ content increase in the films (Monteiro, 2001). The increase of $\mathrm{sp}^{3}$ bonded C in DLC caused by B doping might be associated with formation of B-C bonding. The incorporated $\mathrm{B}$ tends to bond with $\mathrm{C}$ rather than $\mathrm{H}$ in the DLC films to form $\mathrm{sp}^{3}$ carbon neighbours and thus suppresses aromatic $\mathrm{sp}^{2}$ bond formation, considering B-C bond (393 kJ/mol) is stronger than $\mathrm{C}-\mathrm{C}$ bond $(332 \mathrm{~kJ} / \mathrm{mol})$ and $\mathrm{C}-\mathrm{H}$ bond $(414 \mathrm{~kJ} / \mathrm{mol})$ is stronger than $\mathrm{B}-\mathrm{H}$ bond (389 kJ/mol) (He et al., 2000).

\subsection{Synchrotron-based XPS}

To determine the chemical composition of the $\mathrm{B}_{4} \mathrm{C}$ sputtered $\mathrm{B}-\mathrm{C}$ film and B-DLC films 1 - 3 coated on Si substrates, XPS broad scans $(\mathrm{hv}=1000 \mathrm{eV})$ were conducted at SGM beam line, CLS. Figure 4.9 shows the XPS spectra of the as-deposited B-DLC films and B-C film. All the spectra exhibit an oxygen $(\mathrm{O}) 1 \mathrm{~s}$ peak around the binding energy of $532 \mathrm{eV}$, a weak $\mathrm{N}$ 1s peak around the binding energy of $399 \mathrm{eV}$ and an intense $\mathrm{C} 1$ s peak around the binding energy of 284 $\mathrm{eV}$. The detected small amount of $\mathrm{O}$ and $\mathrm{N}$ in those films can be explained by the surface physical absorption (diffusion) after exposure of the deposited films to atmospheric air and/or the long rang ordered $\mathrm{O}$ and $\mathrm{N}$ absorption during film synthesis in the vacuum chamber of the BTIBD system. Due to current equipment limitation, we cannot measure the $\mathrm{O}$ and $\mathrm{N}$ by a new XPS scan after in situ film sputtering cleaning to determine their origin. According to what Tang 
reported on the a-C:H films synthesized using the same BTIBD facility (Tang, 2010), the O and $\mathrm{N}$ are mainly from the film exposure to air after deposition because the $\mathrm{O}$ peak in XPS spectra after Ar ion cleaning has been greatly reduced and the $\mathrm{N}$ peak has almost disappeared. The XPS spectrum of the $\mathrm{B}_{4} \mathrm{C}$ sputtered $\mathrm{B}-\mathrm{C}$ film also displays $\mathrm{B}$ 1s peak located around the binding energy of $188 \mathrm{eV}$ as well as Si 2p peak located around the binding energy of $102.3 \mathrm{eV}$. However, for the B-DLC films neither B nor Si was detected by XPS. The absence of B in the surface is probably due to the deposition procedure, where the $\mathrm{B}_{4} \mathrm{C}$ target sputtering was turned off before the DLC deposition source at the end of deposition. The absence of Si suggests that the B-DLC films are thick enough to overcome the probing depth of the photoelectrons we used.

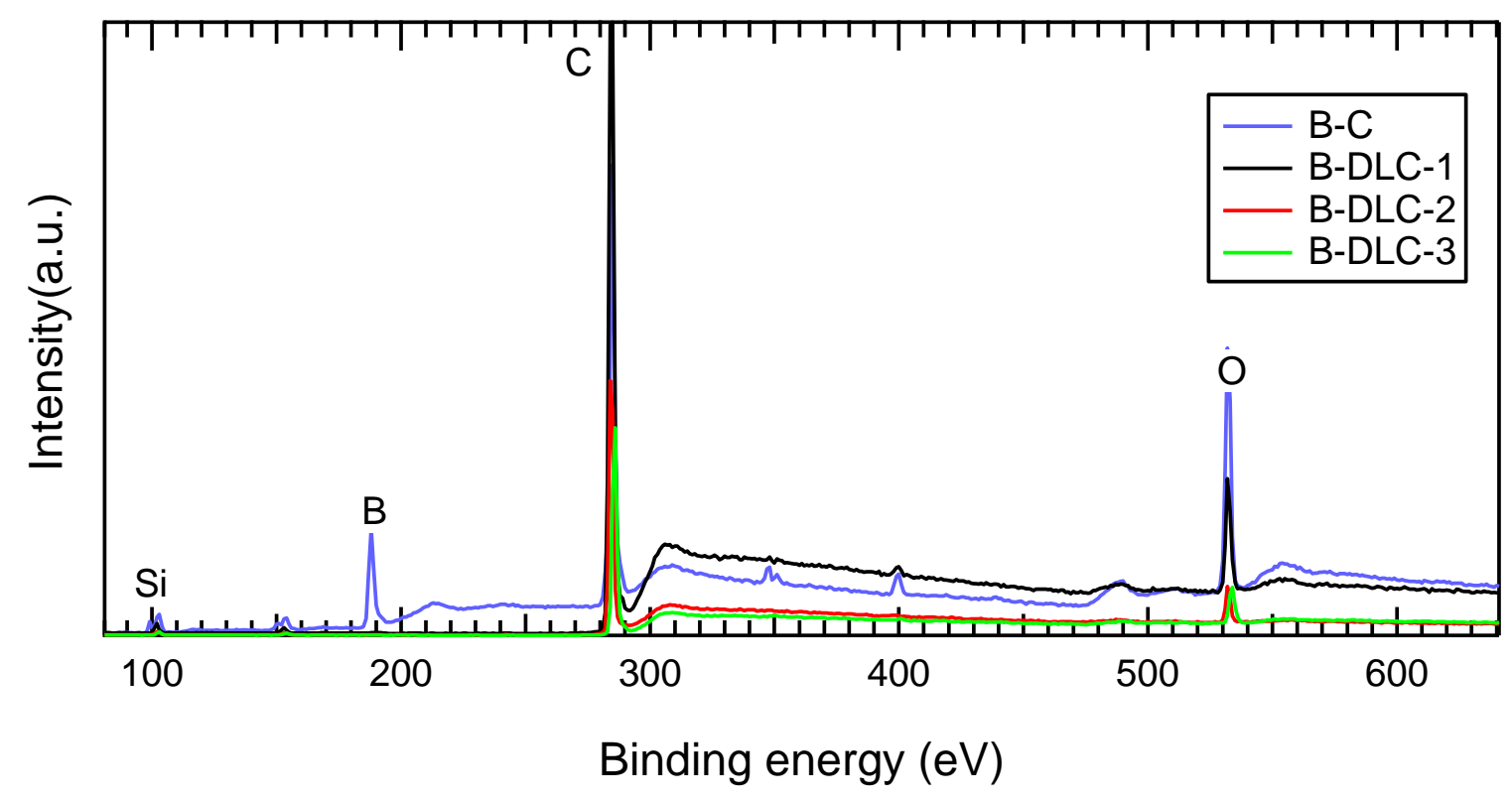

Figure 4.9 XPS spectra of $\mathrm{B}_{4} \mathrm{C}$ sputtered film and B-DLC films

The enlarged XPS spectra of C 1s of the films are shown in Figure 4.10. The C 1s peaks of the spectra are located around the binding energies of 284 and $285 \mathrm{eV}$. The $\mathrm{C} 1 \mathrm{~s}$ spectral line mainly includes the components of $\mathrm{sp}^{2} \mathrm{C}$ bonds at lower binding energy and $\mathrm{sp}^{3} \mathrm{C}$ bonds at higher binding energy. As shown in Figure 4.10, the C 1s peaks of the 3 B-DLC films slightly shift toward low binding energy from B-DLC 3 to B-DLC 1 with the increase of B concentration, 
which is correlated to the decrease of $\mathrm{sp}^{3}$ bonded $\mathrm{C}$ in the films. The result suggests that with the increase of B content in the B-DLC films the $\mathrm{sp}^{3}$ bonded $\mathrm{C}$ in the films seems to decrease. This is consistent with the aforementioned Raman result. Pu et al. reported that from XPS measurements on the B incorporated (up to 26.9 at. \%) hydrogen free DLC films and pure DLC films prepared by radio frequency magnetron sputtering, the obtained C 1s spectra of the B-DLC films slightly shifted toward low binding energy comparing to those of the DLC films, which is associated with the decrease of $\mathrm{sp}^{3} \mathrm{C}$ bonding ( $\mathrm{Pu}$ et al., 2010). Their results show that the B doping leads to the decrease of $\mathrm{sp}^{3} \mathrm{C}$ bonding, which is contrary to the present result, and that the content of $\mathrm{sp}^{3} \mathrm{C}$ bonding decreases with the increase of $\mathrm{B}$ content in the films. These will be further explained in the next session based on the chemical states of B in the films.

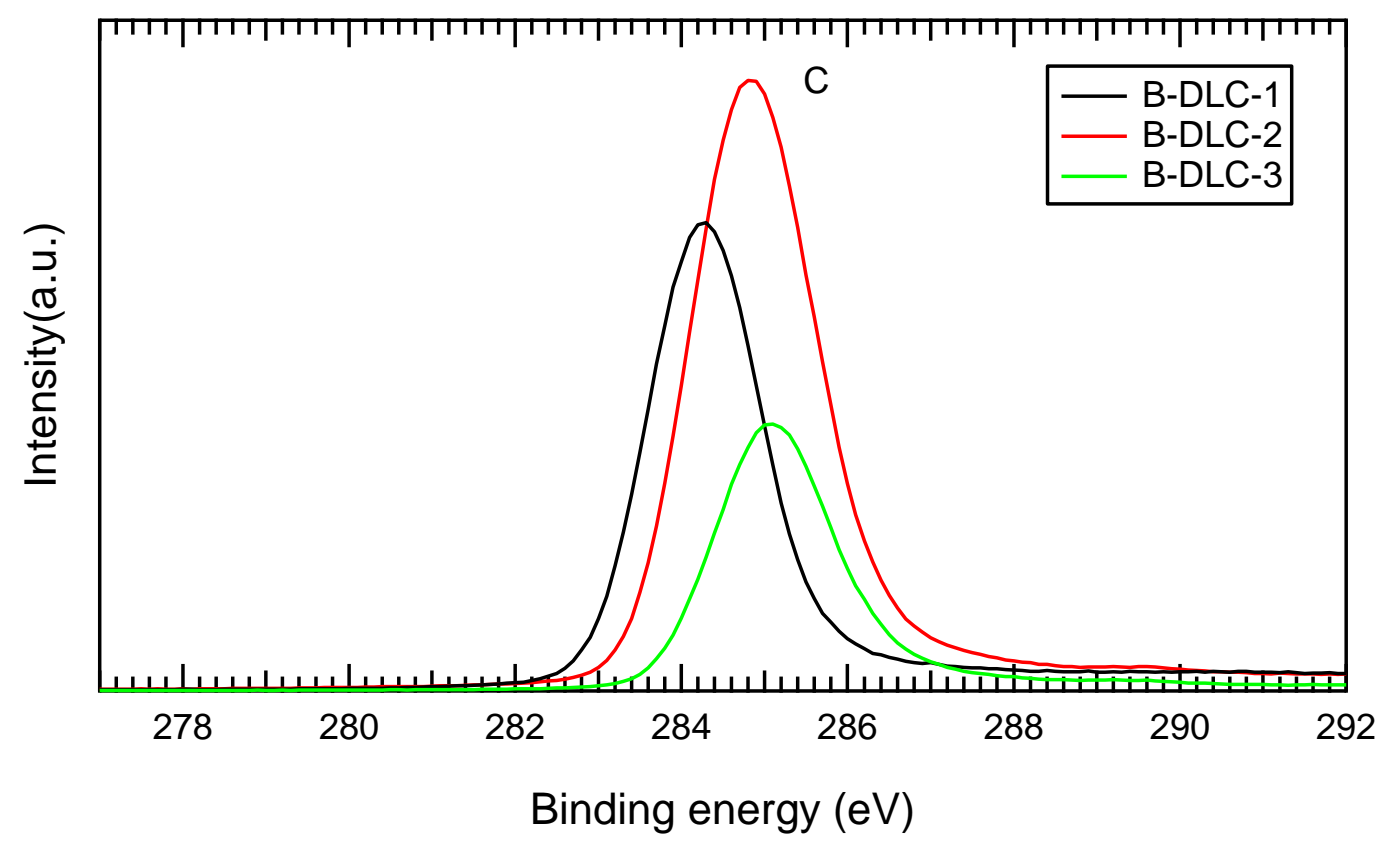

Figure 4.10 XPS spectra of C 1s for B-DLC films

\subsection{Synchrotron-based NEXAFS}

NEXAFS (or XAS) was conducted at SGM beam line, CLS, to investigate the chemical states of B and C in the films. The NEXAFS spectra were collected in both TEY (relatively surface 
sensitive) and FLY (relatively bulk sensitive) modes. Figure 4.11 shows the FLY mode of B K-edge NEXAFS for three B-DLC films and the TEY mode of B K-edge NEXAFS for $\mathrm{B}_{4} \mathrm{C}$ sputtered B-C film. For the B-DLC samples, B can only be detected in FLY mode, not in TEY mode, indicating that there is little B on the surface of the samples. This is consistent with the XPS measurements and further explains why B peak was not present in the XPS spectra (surface sensitive).

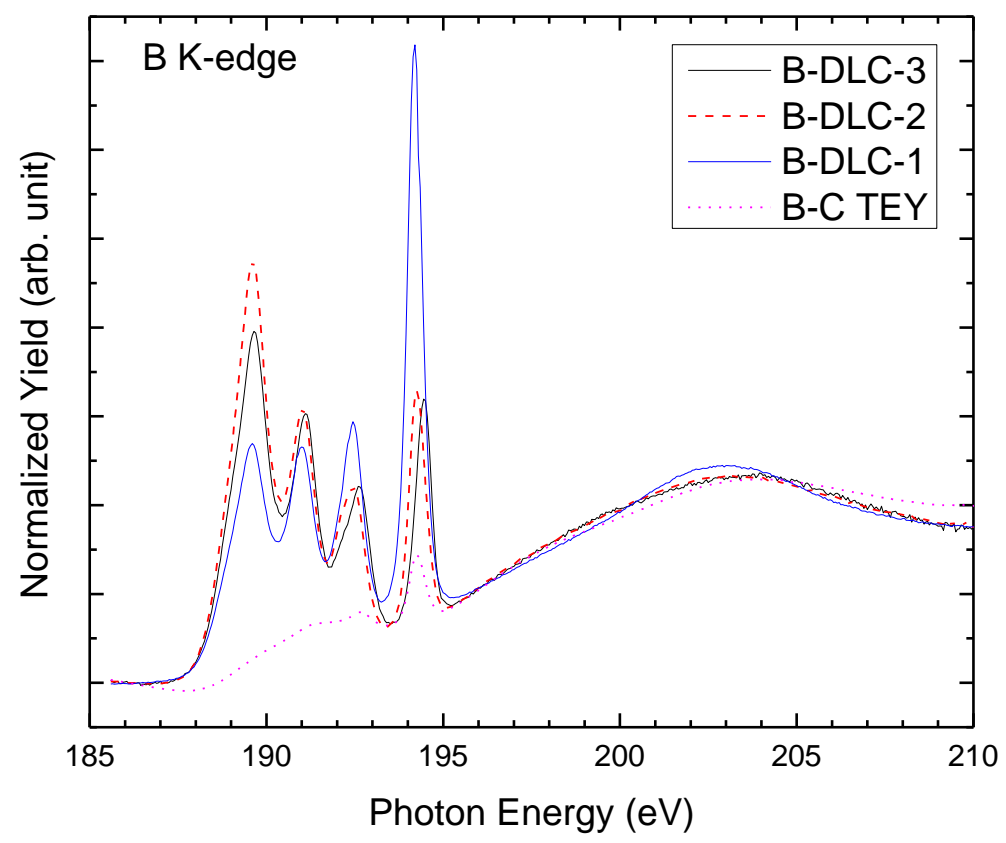

Figure 4.11 B K-edge NEXAFS in FLY mode for B-DLC films and in TEY mode for B-C film As seen from the B K-edge NEXAFS spectra presented in Figure 4.11, 4 peaks located at the photon energies of $189 \mathrm{eV}, 190.6 \mathrm{eV}, 192 \mathrm{eV}$ and $194 \mathrm{eV}$ are distinct for the B-DLC film samples. These 4 peaks can be assigned to C-rich boron carbide, B-rich boron carbide (like $\mathrm{B}_{4} \mathrm{C}$ ), boron suboxide and boron oxide, respectively (Caretti et al., 2008). Thus for the B-DLC films, B exists in four different states including C-rich and B-rich boron carbides, boron suboxide and 
boron oxide. For the $\mathrm{B}_{4} \mathrm{C}$ sputtered $\mathrm{B}-\mathrm{C}$ film, $\mathrm{B}$ exists as boron suboxide, boron oxide, and B-rich boron carbide.

The relative peak intensity of boron oxides is high for the B-C film, indicating that a significant amount of B has been oxidized. The spectrum of B-DLC-1 also displays high intensity ratio of boron oxides to boron carbides, while the spectra of B-DLC-2 and B-DLC-3 show much lower intensity ratios of boron oxides to boron carbides. It appears that higher B concentration in the film leads to higher degree of $\mathrm{B}$ oxidization. The $\mathrm{O}$ in the films can be introduced from both of film exposure to atmospheric air after deposition and moisture, residual air or impurity of carrier gases in the vacuum chamber during film synthesis. Considering that boron oxides were detected in FLY mode, the $\mathrm{O}$ in the films is probably introduced during the deposition. When the $\mathrm{B}_{4} \mathrm{C}$ target was sputtered by $\mathrm{Ar}$ ions with high energy, most of the $\mathrm{B}-\mathrm{C}$ bonds were broken. Since B-O bond $(515 \mathrm{~kJ} / \mathrm{mol})$ is stronger than B-C bond $(393 \mathrm{~kJ} / \mathrm{mol})$, the sputtered atomic or ionic B was apt to bond with the $\mathrm{O}$ in the vacuum chamber. For B-DLC with higher B content, higher target bias voltage and/or higher ion current were used for sputtering of $\mathrm{B}_{4} \mathrm{C}$ and thus more atomic or ionic $\mathrm{B}$ was available to bond with the $\mathrm{O}$, resulting in higher degree of B oxidization. Due to the much higher degree of B oxidation, B-DLC-1 consists of less boron carbides than B-DLC-2 and B-DLC-3 even though its B content is much higher. As for B-DLC-2 and B-DLC-3, they have similar total B content and show similar degree of B oxidation, but B-DLC-2 shows relatively higher content of C-rich carbide and lower content of B-rich carbide than B-DLC-3.

Figure 4.12 and 4.13 show the TEY and FLY modes of C K-edge NEXAFS spectra for B-DLC films and $\mathrm{B}_{4} \mathrm{C}$ sputtered B-C film, respectively. Two main peaks are observed in all the spectra in both modes. The peak located around $286.9 \mathrm{eV}$ corresponds to the transition $\mathrm{C} 1 \mathrm{~s} \rightarrow$ 
$\pi *$ for the $\mathrm{sp}^{2} \mathrm{C}=\mathrm{C}$ bond (Monteiro, 2001). The peak located around $290 \mathrm{eV}$ is the most interesting one and it is associated to the excitons from $\mathrm{C} 1 \mathrm{~s}$ to $\sigma *$ in $\mathrm{sp}^{3}$-rich material (Monteiro, 2001). From B-DLC-3 to B-DLC-1, the intensity of the absorption peak around $290 \mathrm{eV}$ in both modes exhibits a distinct decrease, indicating that B-DLC-3 has the highest content of $\mathrm{sp}^{3}$ bonded $\mathrm{C}$ in the film and B-DLC-1 has the lowest $\mathrm{sp}^{3}$ content. This is probably associated with the oxidization of B. As shown in Figure 4.11, the degree of B oxidation increases with the increase of B content in the film. The actual B content to form B-C bonding is lower for B-DLC-1 than other samples with lower total B content. As we have discussed in Section 4.3, the increase of $\mathrm{sp}^{3}$ bonded $\mathrm{C}$ by $\mathrm{B}$ doping is probably due to the formation of $\mathrm{B}-\mathrm{C}$ bonding (He et al., 2000). Therefore it is reasonable that the $\mathrm{sp}^{3} \mathrm{C}$ bonding content of B-DLC-2 and 3 is higher than B-DLC-1. As for B-DLC-2 and B-DLC-3, B-DLC-2 has slightly lower content of B-rich carbide. Its lower $\mathrm{sp}^{3} \mathrm{C}$ bonding content might be associated with its relatively lower content of B-rich carbide which might more effectively assist $\mathrm{C}$ to form $\mathrm{sp}^{3}$ bonding in the film. Correlating the $\mathrm{sp}^{3}$ content variation trend with the phase content obtained from NEXAFS in this section, it appears that the higher the content of B-rich carbide, the higher the $\mathrm{sp}^{3}$ content in the film.

For the B-C film, the C K-edge NEXAFS spectrum shown in Figure 4.12 exhibits relatively low $\pi^{*}$ peak at $286.9 \mathrm{eV}$ and high $\sigma$ exciton peak at $290 \mathrm{eV}$, indicating the film consists of primarily high fraction of $\mathrm{sp}^{3}$ bonded C. Monteiro did NEXAFS measurements on the $\mathrm{B}_{4} \mathrm{C}$ films prepared by filtered cathodic arc deposition using $\mathrm{B}_{4} \mathrm{C}$ cathode and obtained similar result (Monteiro, 2001). 


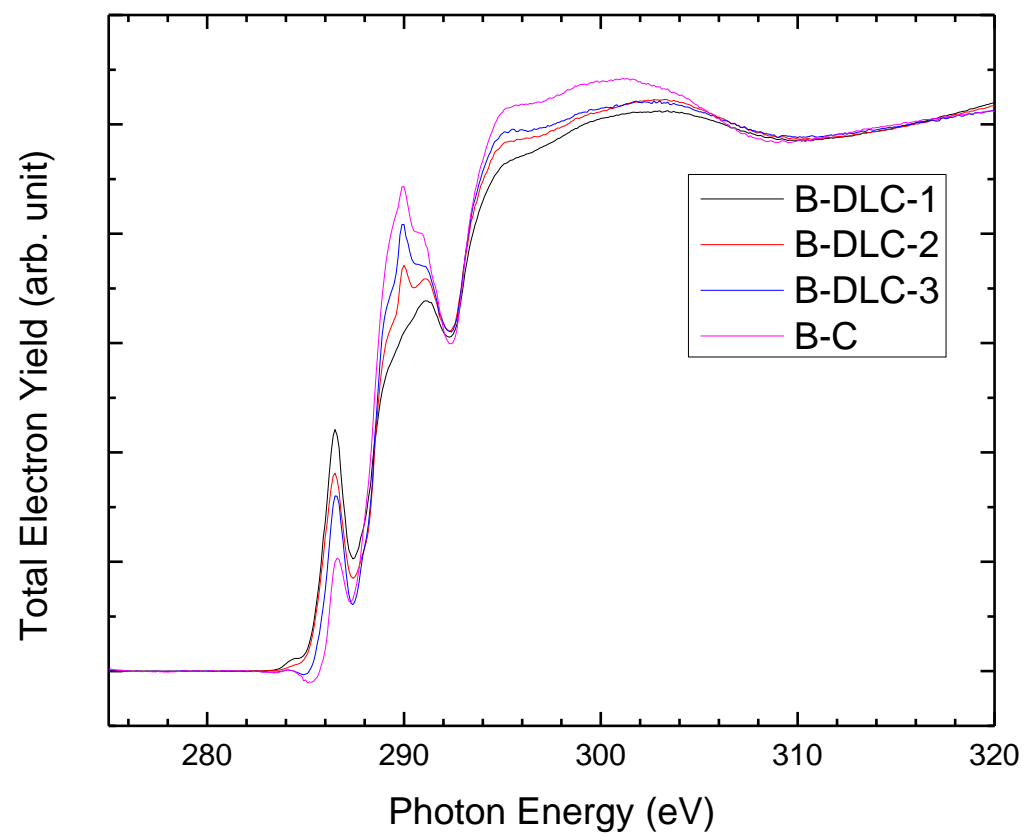

Figure 4.12 C K-edge NEXAFS in TEY mode for B-C film and B-DLC films

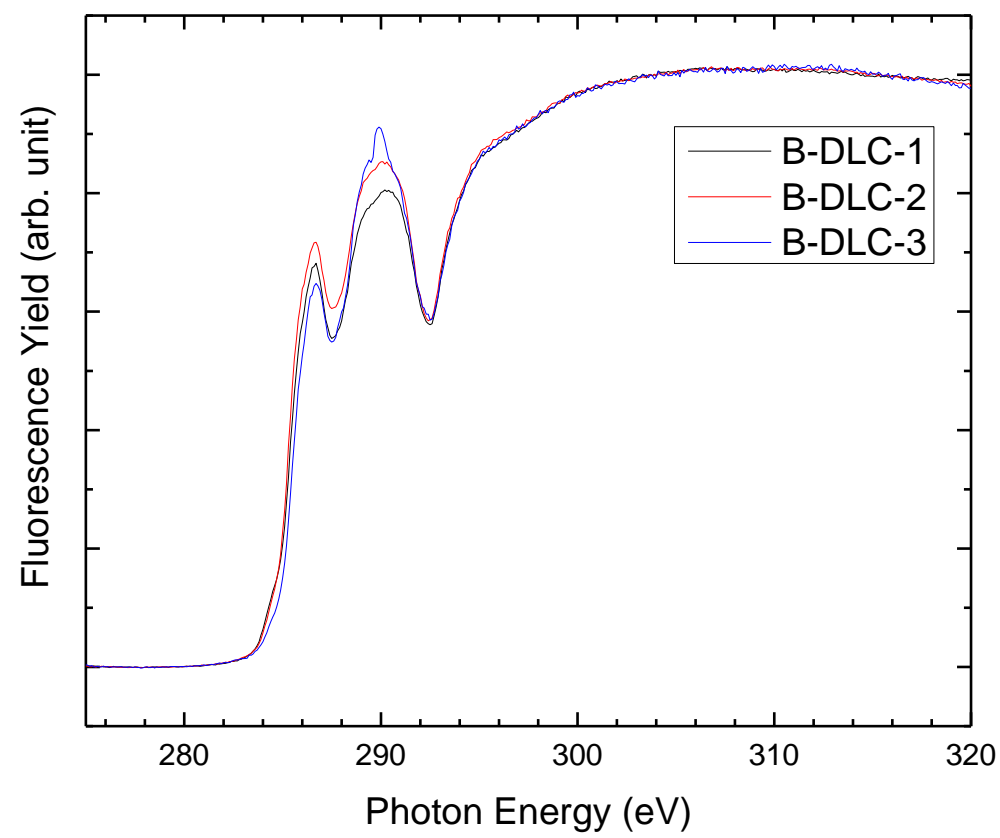

Figure 4.13 C K-edge NEXAFS in FLY mode for B-DLC films 


\subsection{AFM Observation}

DLC coated and B-DLC coated silicon wafers were observed using AFM. The AFM images of film surface topography are shown in Figure 4.14 - 4.17. The AFM measured root mean square (RMS) surface roughness for the DLC and B-DLC films is listed in Table 4.2.

Table 4.2 Measured surface roughness for DLC and B-DLC films

\begin{tabular}{lcccc}
\hline Samples & DLC & B-DLC-1 & B-DLC-2 & B-DLC-3 \\
\hline RMS roughness $(\mathrm{nm})$ & $2.5 \pm 0.4$ & $2.4 \pm 0.2$ & $1.5 \pm 0.1$ & $1.1 \pm 0.1$ \\
\hline Scanned area $(\mu \mathrm{m})$ & $1.5 \times 1.5$ & $1.5 \times 1.5$ & $1.5 \times 1.5$ & $1.5 \times 1.5$
\end{tabular}

The result indicates that B doping into DLC films makes the film surface smoother and the surface roughness of the B-DLC films tends to be lower with the increase of $\mathrm{sp}^{3}$ content in the film, which appears to increase with the increase of B-rich B-C bonding content in the B-DLC films. Comparing B doping with $\mathrm{N}$ doping into DLC films using the same BTIBD system, $\mathrm{N}$ doping also makes the film surface smoother as Sethuraman reported (Sethuraman, 2009). However, Monteiro (Monteiro, 2001) observed that the B-DLC films deposited by filtered cathodic arc deposition are significantly rougher than the reference DLC film. This is probably attributed to the particulates generated from the arc discharge not being filtered well for deposition and undermining surface roughness. On the contrary, BTIBD is an intrinsically particulate-free technique. 


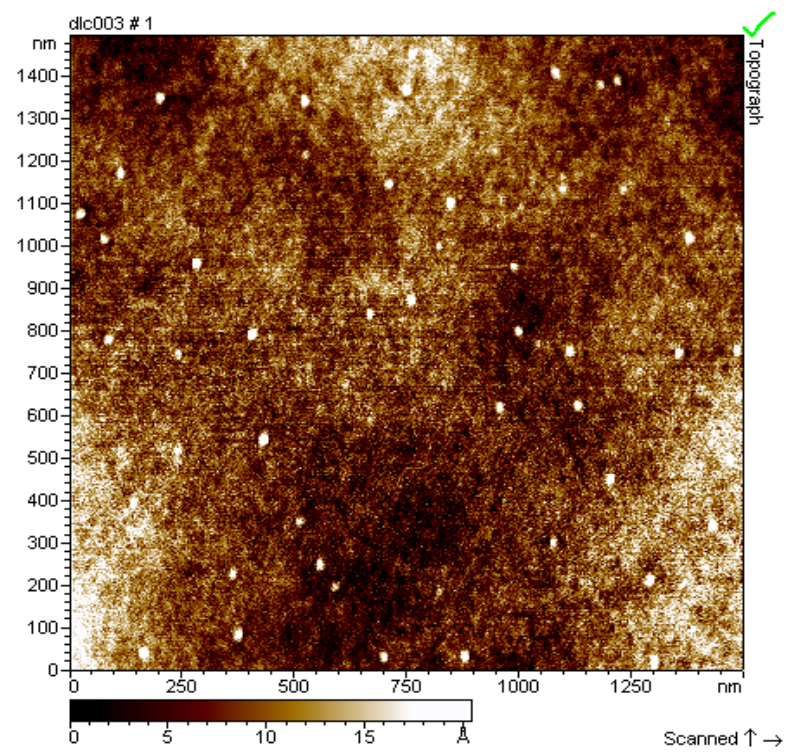

Figure 4.14 AFM image of DLC film (Si wafer 3)

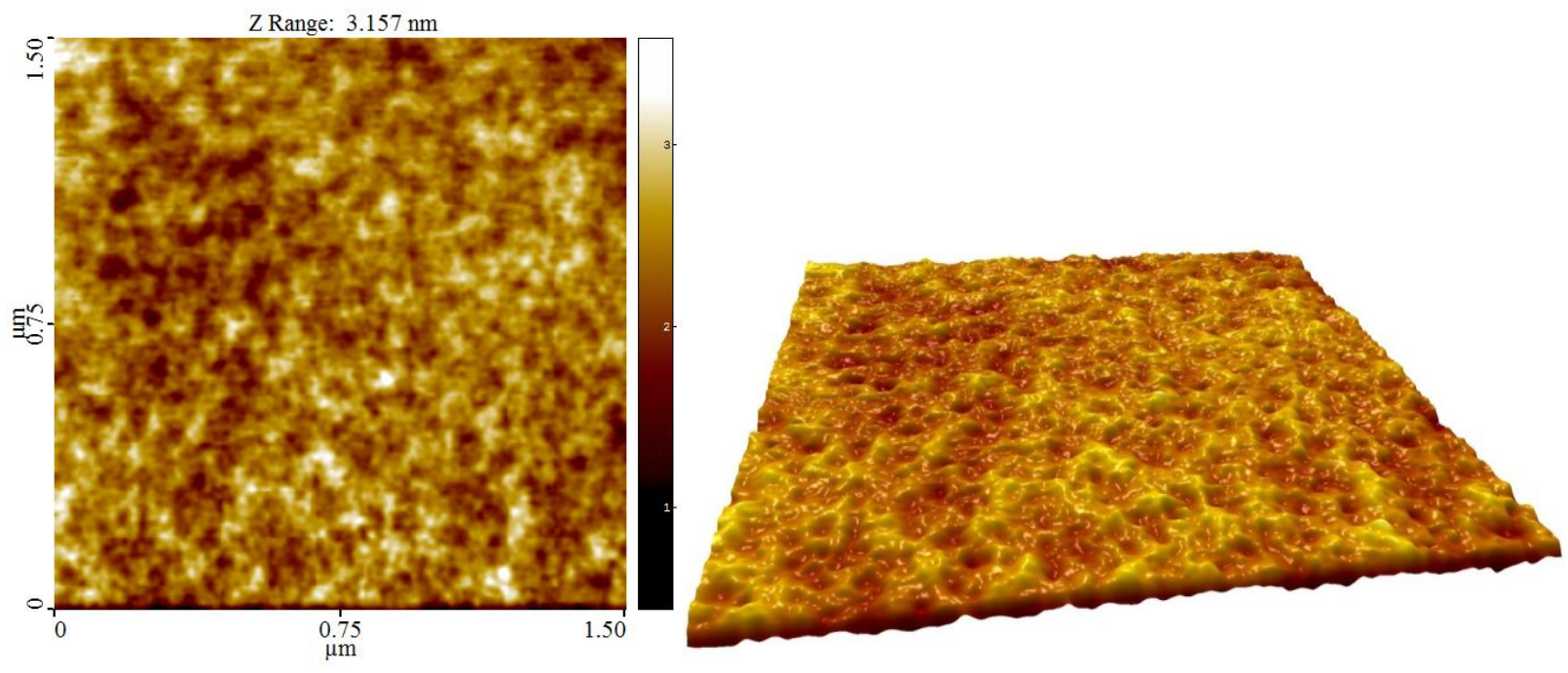

Figure 4.15 AFM images of B-DLC-1 


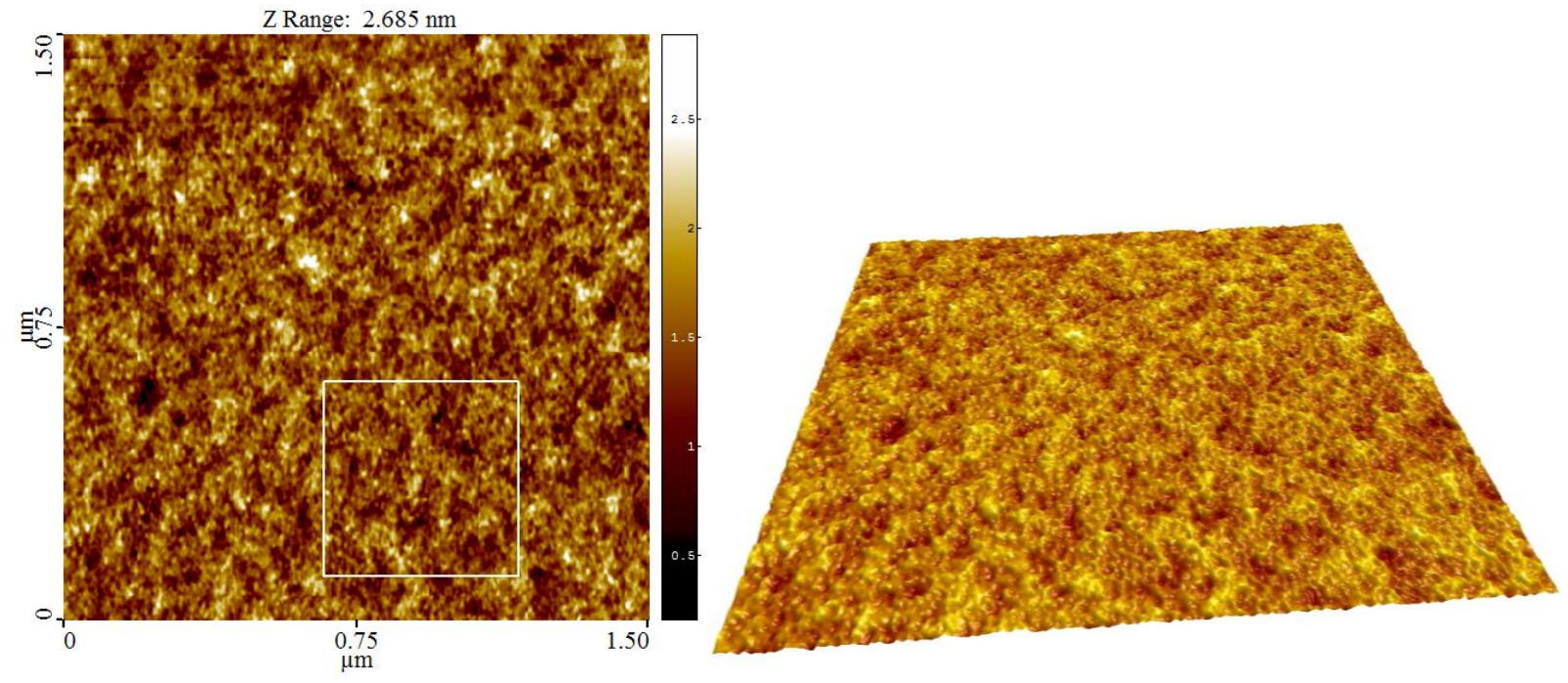

Figure 4.16 AFM images of B-DLC-2

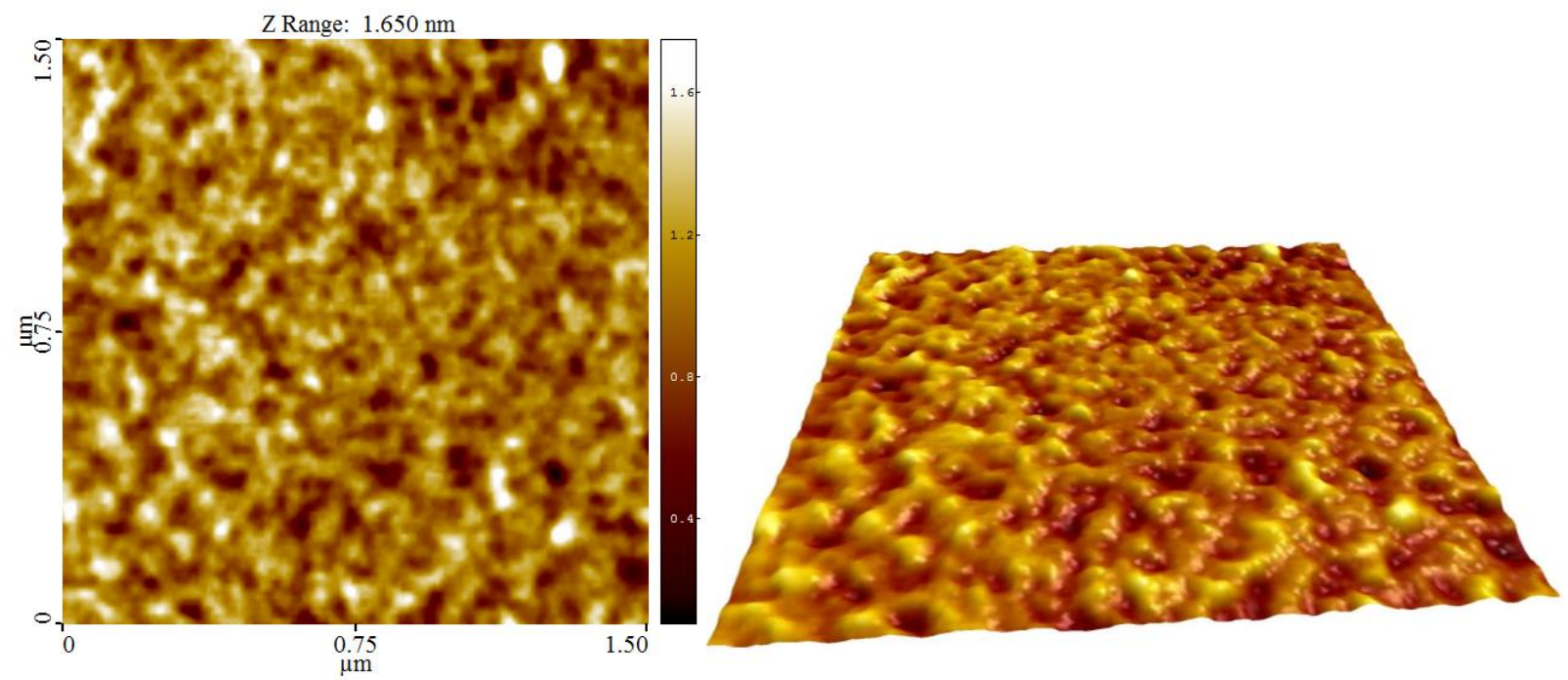

Figure 4.17 AFM images of B-DLC-3

Both the DLC and B-DLC films exhibit very smooth surface and good homogeneity. According to what Maheswaran et al. reported (Maheswaran et al., 2011), DLC film growth includes three distinct stages, i.e. nucleation, island growth and formation of continuous film. The nucleation starts at the subsurface or the surface of the substrate depending on different impinging ion energies (high to low), and diffuses across the substrate surface leading to 
formation of $\mathrm{sp}^{3}$ and $\mathrm{sp}^{2}$ hybridizations. The islands occur by bonding of adatoms, ripening of clusters and/or coalescence of neighbor islands. When the islands grow in size, all of them combine with their neighbors to form a continuous film. High nucleation density over the whole substrate surface and well-controlled ion energy are essential to form smooth and homogeneous DLC films (Tang, 2010).

It's reported that B-DLC thin films are constituted by B nanoparticles dispersed and embedded in the DLC matrix (Sikora et al., 2009a). The B doping into DLC helps smoothen the film surface and the reason may be the B nanoparticles reducing the surface energy of DLC. Lower surface energy reduces nucleation barrier, leading to higher nucleation density, and thus lowers surface roughness.

\subsection{Mechanical Properties}

Three nanoindentation tests were performed to measure the hardness and elastic modulus of Oliver and Pharr method for each film sample under a load of $0.5 \mathrm{mN}$. The results are presented in Figure 4.18. The average hardness and elastic modulus of DLC film are $11 \mathrm{GPa}$ and $73 \mathrm{GPa}$, respectively. The average hardness of B-DLC-1, 2 and 3 is 12, 13, $14 \mathrm{GPa}$, respectively and their corresponding elastic modulus is $84,90,97 \mathrm{GPa}$, respectively. Both the hardness and elastic modulus of B-DLC films are higher than those of DLC film, showing that the incorporation of B to DLC results in the increase of both hardness and elastic modulus. Furthermore, both hardness and elastic modulus increase with the increase of $\mathrm{sp}^{3}$ content in the film, which appears to increase with the increase of B-rich B-C bonding content in the B-DLC films.

It's generally accepted that the lower hardness of DLC comparing to diamond is because of the existence of $\mathrm{sp}^{2}$ bonded carbon as well as hydrogen in DLC and the hardness of DLC has a close relationship with its $\mathrm{sp}^{3} / \mathrm{sp}^{2}$ ratio, that is, the higher $\mathrm{sp}^{3} / \mathrm{sp}^{2}$ ratio brings the higher hardness 
(Robertson, 1993). In this thesis work, the results from Raman, XPS and NEXAFS confirm that the B-DLC films have higher $\mathrm{sp}^{3} / \mathrm{sp}^{2}$ ratio than the pure DLC films and for the B-DLC films the $\mathrm{sp}^{3} / \mathrm{sp}^{2}$ ratio appears to increase with the increase of B-rich B-C bonding content. Thus it's reasonable that the B incorporation to DLC leads to the increase of both hardness and elastic modulus and for the B-DLC films both hardness and elastic modulus increase with the increase of B-rich B-C bonding content. He et al. in 2000 measured the hardness of the B-DLC films produced by radio frequency plasma-immersion ion deposition with B contents up to 10 at. $\%$ and obtained similar results: the B incorporation to DLC induces an increase of $\mathrm{sp}^{3} \mathrm{C}$ bonding in the films, and thus an increase of hardness, as well as a decrease of stress (He et al., 2000).

(a)
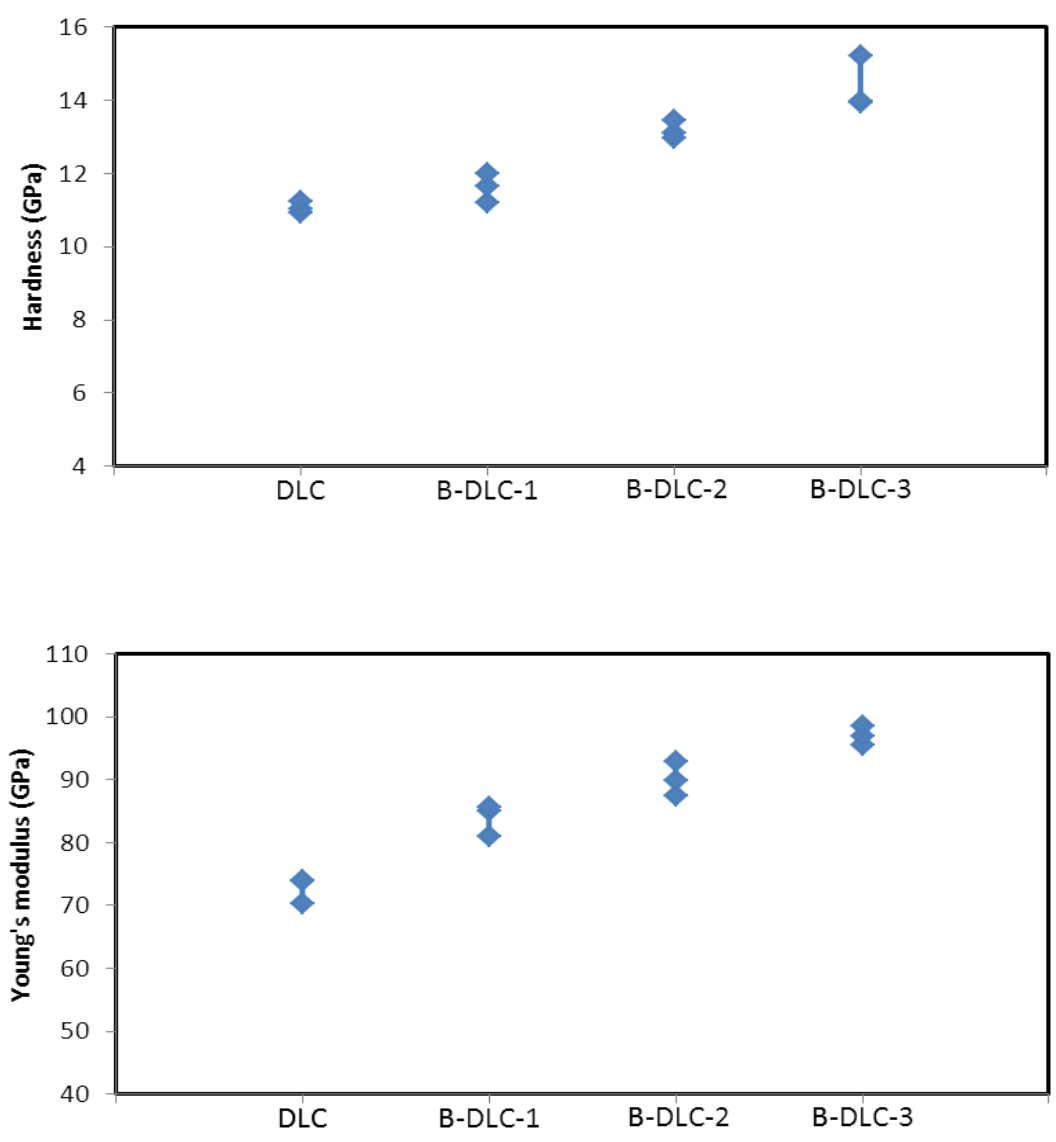

(b)

Figure 4.18 (a) Hardness and (b) Young's modulus of DLC and B-DLC films 
The hardness and elastic modulus values collected from nanoindentation testing on thin films are substrate-independent when the indent depth is under $10 \%$ of the film thickness (Gitis et al., 2008). The indent depth recorded for the nanoindentation testing in this work is from $40 \mathrm{~nm}$ to $43 \mathrm{~nm}$, which is over $10 \%$ of the DLC and B-DLC film thickness (about $0.24 \mu \mathrm{m}$ ). Based on the current state of nanoindenter, the measurement values of the hardness and elastic modulus of Oliver and Pharr method are repeatable and reliable (not affected by noises, waves, etc.) only when the indent depth is deeper than $40 \mathrm{~nm}$, so we could not reach smaller indents under $10 \%$ of the total film thickness to get substrate-independent values. In this case, the Si substrate could influence the hardness and elastic modulus measurement values a little, but the relative values and variation trend would not be influenced based on the same substrate material and measuring conditions.

\subsection{Friction Performance}

Reciprocal ball-on-disc friction tests were performed in normal ambient air under a constant load to measure the COF of the samples, where a stainless steel ball was sliding on Si wafers (without any film as reference), DLC films as well as B-DLC films coated on Si wafers. Three tests at different locations were conducted on each sample. The obtained data of COF are the mean values from the friction tests at different locations of the samples. Figure 4.19 presents the $\mathrm{COF}$ of the samples. It can be clearly seen that the Si wafer has significantly higher COF values comparing to both the DLC and B-DLC films coated on Si wafers. 


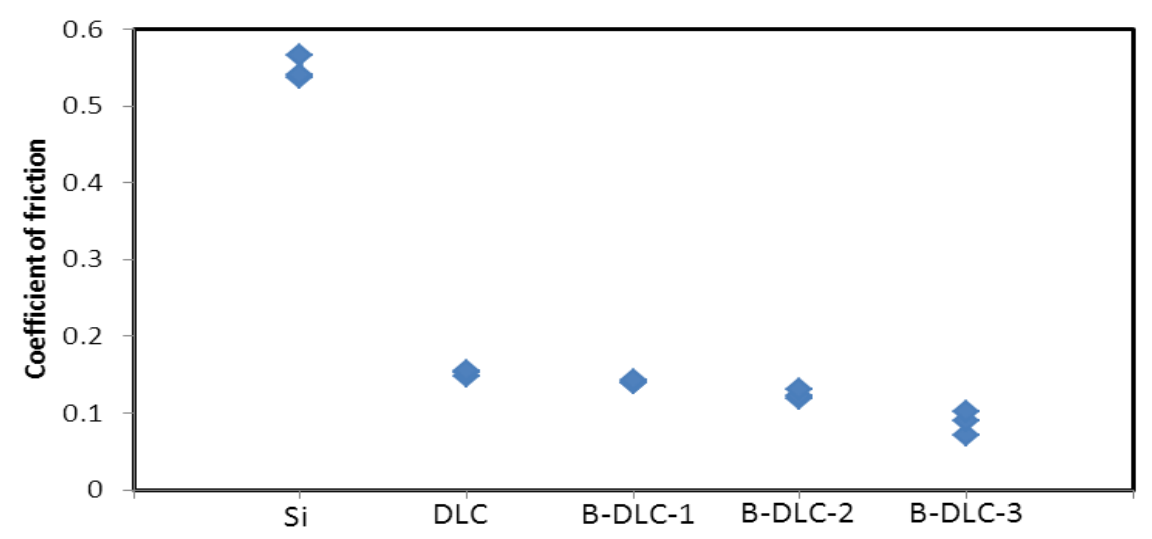

Figure 4.19 COF of Si, DLC film and B-DLC films

The COF values of the DLC film fluctuate around 0.15, which are higher than those of all the B-DLC films, suggesting that the B doping lowers the COF of DLC. Monteiro reported that the COF of hydrogen free DLC deposited by filtered cathodic arc is between 0.1 and 0.2 (Monteiro, 2001), which is comparable to our DLC COF. The values of COF for the B-DLC films vary from 0.07 to 0.14 , which are related to the $\mathrm{sp}^{3}$ (B-rich B-C bonding) content in the samples. The higher the $\mathrm{sp}^{3}$ (B-rich B-C bonding) content, the lower the COF values. The obtained low $\mathrm{COF}$ and its variation trend for the DLC and B-DLC films are in accord with the surface roughness variation trend reported in Section 4.6 (AFM observation) for these films, so it's reasonable to suggest that the surface roughness has a strong influence on film friction coefficient.

According to what Robertson published (Robertson, 2002), the friction behavior of a-C:H films under low humidity can be explained by the contact between two basically similar hydrophobic a-C:H surfaces with very low $\mathrm{COF}$, which are the original hydrophobic a-C:H film and the hydrophobic transfer layer of a-C:H formed on the surface contacting with the a-C:H film. Also, it's pointed out that the covalent Si doping to a-C:H films improves their friction behavior and the reason is that the doped $\mathrm{Si}$ changes the nature of the transfer layer to form a silica-gel-like sacrificial layer (Robertson, 2002). In this work, part of the doped B forms 
covalent bonding with $\mathrm{C}$, the lower $\mathrm{COF}$ of the B-DLC films might be associated to the modification of transfer layer by B doping similar to the case for Si doping. In addition, the lower roughness of B-DLC may also contribute to their lower COF. 


\section{CHAPTER 5 \\ SUMMARY, CONCLUSIONS AND FUTURE WORK}

\subsection{Summary and Conclusions}

The B incorporated DLC thin films were synthesized using a newly developed BTIBD technique. The structure and properties of the synthesized B-DLC films were characterized by various advanced techniques including SEM, AFM, XRD, Raman spectroscopy, synchrotron-based XPS and NEXAFS, nanoindentation and ball-on-disk testing. The summary and conclusions are as follows:

1. B-DLC thin films with different B doping levels (up to 8 wt. \%) have been successfully synthesized by controlling the sputtering parameters of $\mathrm{B}_{4} \mathrm{C}$ target including target bias voltage and ion beam current density. B concentration incorporated increases with the increase of target bias voltage and ion current density.

2. Mass changes of samples before and after deposition were used to estimate film thickness and deposition rate in order to choose appropriate processing conditions to control the $\mathrm{B}$ doping levels. The estimated values were validated by cross-section SEM observation and profilometer measurements.

3. In the B-C films by $\mathrm{B}_{4} \mathrm{C}$ sputtering, the $\mathrm{B}_{4} \mathrm{C}$ phase purity is not retained after ion beam sputtering and boron suboxide, boron oxide as well as B-rich boron carbide are contained. The $\mathrm{C}$ in the $\mathrm{B}-\mathrm{C}$ films is primarily $\mathrm{sp}^{3}$ bonded.

4. In the B-DLC films, B exists in different states including C-rich boron carbide, B-rich boron carbide, boron suboxide and boron oxide. The oxidation of B probably occurs during the film deposition due to the residential oxygen in the vacuum or oxygen impurity from the input gases. 
5. The incorporation of B to DLC leads to the increase of $\mathrm{sp}^{3}$ bonded $\mathrm{C}$ in the films, and thus increased hardness and Young's modulus of the films.

6. The B incorporation to DLC results in the decrease of film surface roughness, and thus decreased friction coefficient.

7. For the B-DLC films, the fraction of $\mathrm{sp}^{3}$ bonded $\mathrm{C}$, the hardness and elastic modulus increase with the increase of B-rich B-C bonding content in the films, whereas the surface roughness and friction coefficient decrease with the increase of B-rich B-C bonding content in the films.

These results demonstrate that the BTIBD technique is promising to produce B-DLC thin films with improved properties for practical applications where film uniformity and smoothness cannot be met by traditionally used sputtering methods.

\subsection{Recommendations for Future Work}

Possible future research related to this project is suggested as follows:

1. Synthesis and characterization of B-DLC with more different B doping levels is desirable to understand the effect of B concentration on the structure and properties of the films.

2. Systematic research on the relationships between processing, structure and properties is desirable in order to develop B-DLC films with desired film structure and properties through manipulating processing conditions for more practical applications.

3. More studies on the B oxidization during film deposition are needed in order to control oxidation.

4. Deposition of B-DLC thin films on different substrates and investigation of their adhesion strength and other properties like biocompatibility would be desirable for their practical applications. 
5. $\mathrm{H}$ content in B-DLC films would be desirable to be determined to study the $\mathrm{H}$ effects on the film structure and properties.

6. New C source of hydrocarbon gas like acetylene and new B source like pure B target may be used to adjust the composition of B-DLC films to obtain different properties.

7. More detailed investigations of tribological properties of B-DLC films under various conditions to get a deep understanding of the friction and wear mechanism should be of great scientific and technical interest. 


\section{LIST OF REFERENCES}

Ahmad A.A., A.M. Alsaad. 2007. Adhesive B-doped DLC films on biomedical alloys used for bone fixation. Bull. Mater. Sci. 30: 301-308

Ahmed S.F., M.K. Mitra, K.K. Chattopadhyay. 2007. The effect of fluorine doping and temperature on the field emission from diamond-like carbon films. Journal of physics Condensed matter 19346233

Aisenberg S., R. Chabot. 1971. Ion-beam deposition of thin films of diamond-like carbon. Journal of Applied Physics 42 (7): 2953-2958

Byon E.S., J.K. Kim, S. Lee, J.H. Hah, K. Sugimoto. 2003. Mechanical properties of B-C-N films deposited by dual cesium ion beam sputtering system. Surface and Coatings Technology 169-170: 340-343

Caretti I., R. Gago, J.M. Albella, I. Jiménez. 2008. Boron carbides formed by coevaporation of B and $\mathrm{C}$ atoms: Vapor reactivity, $\mathrm{B}_{\mathrm{x}} \mathrm{C}_{1-\mathrm{x}}$ composition, and bonding structure. The American Physical Society Physical Review B 77, 174109

Cheng H.F., F.Y. Chuang, C.H. Tsai, W.C. Wang, C.M. Huang, I.N. Lin. 1999. Boron-doping effect on the field emission behavior of pulse laser deposited diamond-like carbon films. Applied Surface Science 142: 504-509

Chen H.Y., J. Wang, H. Yang, W.Z. Li, H.D. Li. 2000. Synthesis of boron carbide films by ion beam sputtering. Surface and Coatings Technology 128-129: 329-333

Dai M.J., K.S. Zhou, S.S. Lin, H.J. Hou, X.G. Zhu, H.W. Li, S.C. Niu. 2007. A Study on metal-doped diamond-like carbon film synthesized by ion source and sputtering technique. Plasma Processes and Polymers 4: S215-S219

Erdemir A., C. Donnet. 2006. Tribology of diamond-like carbon films: recent progress and future prospects. Journal of Physics D: Applied Physics 39: R311-R327

Ferrari A.C., J. Robertson. 2000. Interpretation of Raman spectra of disordered and amorphous carbon. The American Physical Society PRB 61(14): 95-107 
Fu Ricky K.Y., Y.F. Mei, M.Y. Fu, X.Y. Liu, Paul K. Chu. 2005. Thermal stability of metal-doped diamond-like carbon fabricated by dual plasma deposition. Diamond and Related Materials 14: 1489-1493

Gitis N., I. Hermann, S. Kuiry. 2008. Nano and micro indentation and scratch tests of mechanical properties of thin films. Proceedings of the $7^{\text {th }}$ International Conference "The" Coatings in Manufacturing Engineering: 371-378

Hasebe T., S. Nagashima, A. Kamijo, T. Yoshimura, T. Ishimaru, Y. Yoshimoto, S. Yohena, H. Kodama, A. Hotta, K. Takahashi, T. Suzuki. 2007. Depth profiling of fluorine-doped diamond-like carbon (F-DLC) film: Localized fluorine in the top-most thin layer can enhance the non-thrombogenic properties of F-DLC. Thin Solid Films 516: 299-303

He X.M., K.C. Walter, M. Nastasi. 2000. Plasma-immersion ion-processed boron-doped diamond-like carbon films. Journal of Physics Condens. Matter 12: L183-L189

He X., W. Li, H. Li. 1996. Diamond-like carbon film synthesized by ion beam assisted deposition and its tribological properties. Journal of Vacuum Science and Technology A 14 (4): 2039-2047

Hylton T.L., B. Ciorneiu, D.A. Baldwin, O. Escorcia, J. Son, M.T. McClure, G. Waters. 2000. Thin film processing by biased target ion beam deposition. IEEE Transactions on Magnetics 36 (5): 2966-2971

Kahn M., N. Menegazzo, B. Mizaikoff, R. Berghauser, J.M. Lackner, D. Hufnagel, W. Waldhauser. 2007. Properties of DLC and nitrogen-doped DLC films deposited by DC magnetron sputtering. Plasma processes and polymers 4: S200-S204

Kautek W., S. Pentzien, A. Conradi, J. Kruger, K.W. Brzezinka. 1996. Pulsed-laser deposition and boron-blending of diamond-like carbon (DLC) thin films. Applied Surface Science 106: $158-165$

Khadro B., A. Sikora, A.S. Loir, A. Errachid, F. Garrelie, C. Donnet, N. Jaffrezic- Renault. 2011. Electrochemical performances of $\mathrm{B}$ doped and undoped diamond-like carbon (DLC) films deposited by femtosecond pulsed laser ablation for heavy metal detection using square wave anodic stripping voltammetric (SWASV) technique. Sensors and Actuators B 155: 120-125 
Lifshitz Y. 1996. Hydrogen-free amorphous carbon films: correlation between growth conditions and properties. Diamond and Related Materials 5: 388-400

Lifshitz Y. 1999. Diamond-like carbon - present status. Diamond and Related Materials 8: $1659-1676$

Lifshitz Y. 2003. Pitfalls in amorphous carbon studies. Diamond and Related Materials 12: $130-140$

Lui C., W. Gou, G. Li, L. Wang, Z. Mu. 2007. Improvement of mechanical properties of diamond-like carbon film by Ti ion implantation. Plasma Processes and Polymers 4: S265-S268

Maheswaran R., S. Ramaswamy, D. J. Thiruvadigal, C. Gopalakrishnan. 2011. Systematic study of various stages during the growth process of diamond-like carbon film by atomic force microscopy. Journal of Non-Crystalline Solids 357: 1710-1715

Maitz M.F., R. Gago, B. Abendroth, M. Camero, I. Caretti, U. Kreissig. 2006. Hemocompatibility of low-friction boron-carbon-nitrogen containing coatings. Journal of Biomedical Materials Research Part B: Applied Biomaterials 77B: 179-187

Monteiro O.R. 2001. Synthesis, properties and applications of pure and covalently doped DLC films prepared by energetic condensation. Lawrence Berkeley National Laboratory

Nakazawa H., A. Sudoh, M. Suemitsu, K. Yasui, T. Itoh, T. Endoh, Y. Narita, M. Mashita. 2010. Mechanical and tribological properties of boron, nitrogen- coincorporated diamond-like carbon films prepared by reactive radio-frequency magnetron sputtering. Diamond \& Related Materials 19: 503-506

Oliver W.C., G.M. Phar. 2004. Measurement of hardness and elastic modulus by instrumented indentation: Advances in understanding and refinements to methodology. Journal of Materials Research 19: 3-20

Park K.C., J.H. Moon, J.G. Kim, S.J. Chung, M.H. Oh, J. Jang. 1996. Field emission of nitrogen doped DLC films deposited by PECVD. 9th International Vacuum Microelectronics Conference, Technical Digest: 263-267 
Podgornik B., D. Hren, J. Vižintin. 2005. Low-friction behaviour of boundary-lubricated diamond-like carbon coatings containing tungsten. Thin Solid Films 476: 92-100

Pu J.C., S.F. Wang, C.L. Lin, J.C. Sung. 2010. Characterization of boron-doped diamond-like carbon prepared by radio frequency sputtering. Thin Solid Films 519: 521-526

Robertson J. 1986. Amorphous carbon. Advances in Physics 35: 317-374

Robertson J. 1993. Deposition mechanisms for promoting $\mathrm{sp}^{3}$ binding in diamond-like carbon. Diamond and Related Materials 2: 984-989

Robertson J. 1994. The deposition mechanism of diamond-like a-C and a-C:H. Diamond and Related Materials 3: 361-368

Robertson J. 2002. Diamond-like amorphous carbon. Materials Science and Engineering R 37: 129-281

Ronning C., U. Griesmeier, M. Gross, H.C. Hofsgss, R.G. Downing, G.P. Lamaze. 1995. Conduction processes in boron- and nitrogen-doped diamond-like carbon films prepared by mass-separated ion beam deposition. Diamond and Related Materials 4: 666-672

Sethuraman S. 2009. Ion beam deposition of nitrogen doped diamond-like carbon thin films for enhanced biological properties. M.Sc. Thesis, University of Saskatchewan

Shirley D.A. 1972. High-resolution x-ray photoemission spectrum of the valence bands of gold. Physical Review B 5(12): 4709

Sikora A., A. Berkesse, O. Bourgeois, J.L. Garden, C. Guerret-Pie'court, J.N. Rouzaud, A.S. Loir, F. Garrelie, C. Donnet. 2009a. Structural and electrical characterization of boron-containing diamond-like carbon films deposited by femtosecond pulsed laser ablation. Solid State Sciences 11: 1738-1741

Sikora A., O. Bourgeois, J.C. Sanchez-Lopez, J.N. Rouzaud, T.C. Rojas, A.S. Loir, J.L. Garden, F. Garrelie, C. Donnet. 2009b. Effect of boron incorporation on the structure and electrical properties of diamond-like carbon films deposited by femtosecond and nanosecond pulsed laser ablation. Thin Solid Films 518: 1470-1474 
Tamor M.A., W.C. Vassell. 1994. Raman "fingerprinting" of amorphous carbon films. Journal of Applied Physics 76(6): 3823

Tang Y. 2010. Plasma and ion beam enhanced chemical vapour deposition of diamond and diamond-like carbon. Ph.D. Thesis, University of Saskatchewan

Tang Y., Y.S. Li, C.Z. Zhang, J. Wang, Q. Yang, A. Hirose. 2011a. Synthesis of cobalt/diamond-like carbon thin films by biased target ion beam deposition. Diamond \& Related Materials 20: 538-541

Tang Y., Y.S. Li, Q. Yang, A. Hirose. 2011b. Characterization of hydrogenated amorphous carbon thin films by end-Hall ion beam deposition. Applied Surface Science 257: 4699-4705 Tougaard S. 1988. Quantitative analysis of surface electron spectra: Importance of electron transport. Surface and Interface Analysis 11(9): 453-472

Ulrich S., A. Kratzsch, H. Leiste, M. Stüber, P. Schlossmacher, H. Holleck, J. Binder, D. Schild, S. Westermeyer, P. Becker, H. Oechsner. 1999. Variation of carbon concentration, ion energy, and ion current density of magnetron-sputtered boron carbonitride films. Surface \& Coatings Technology 116-119: 742-750

Ulrich S., J. Ye, M. Stüber, C. Ziebert. 2009. Cubic boron nitride based metastable coatings and nanocomposites. Thin Solid Films 518: 1443-1450

Vercammen K., K. VanAcker, A. Vanhulsel, J. Barriga, A. Arnsek, M. Kalin, J. Meneve. 2004. Tribological behaviour of DLC coatings in combination with biodegradable lubricants. Tribology International 37: 983-989

VijaiBharathy P., D. Nataraj, D. Mangalaraj, M.S.R.N. Kiran, J. Silvestre-Albero, Q. Yang. 2011. Influence of tungsten content in W-DLC nanocomposite thin films prepared by hybrid target biased ion beam assisted deposition technique. International Journal of Nanoscience 10: 851-855

VijaiBharathy P., D. Nataraj, P.K. Chu, H. Wang, Q. Yang, M.S.R.N. Kiran, J. Silvestre-Albero, D. Mangalaraj. 2010a. Effect of titanium incorporation on the structural, mechanical and biocompatible properties of DLC thin films prepared by reactive-biased target ion beam deposition method. Applied Surface Science 257: 143-150 
VijaiBharathy P., Y.Y. Chang, D. Nataraj, Q. Yang, S.M. Yang, D. Mangalaraj, L. Yang, T.J. Webster. 2010b. Effect of nickel incorporation on structural, nanomechanical and biocompatible properties of amorphous hydrogenated carbon thin films prepared by low energy biased target ion beam deposition. Thin Solid Films 519: 1623-1628

Wang D.Y., K.W. Weng, S.Y. Hwang. 2000. Study on metal-doped diamond-like carbon films synthesized by cathodic arc evaporation. Diamond and Related Materials 9: 1762-1766

Wang F.M., M.W. Chen, Q.B. Lai. 2010. Metallic contacts to nitrogen and boron doped diamond-like carbon films. Thin Solid Films 518: 3332-3336

Wolf B. 1995. Handbook of ion sources. CRC Press p. 222

Wu Y.H., C.M. Hsu, C.T. Chia, I.N. Lin, C.L. Cheng. 2002. Field emission and Raman spectroscopy studies of atomic hydrogen etching on boron and nitrogen doped DLC films. Diamond and Related Materials 11: 804-808

Yan X., T. Xu, G. Chen, Sh. Yang, H. Liu. 2004. Study of structure, tribological properties and growth mechanism of DLC and nitrogen-doped DLC films deposited by electrochemical technique. Applied Surface Science 236: 328-335

Yao Zh.Q., P. Yang, N. Huang, H. Sun, J. Wang. 2004. Structural, mechanical and hydrophobic properties of fluorine-doped diamond-like carbon films synthesized by plasma immersion ion implantation and deposition (PIII-D). Applied Surface Science 230: 172-178

Yasui H., Y. Hirose, K. Awazu, M. Iwaki. 2000. The properties of BCN films formed by ion beam assisted deposition. Colloids and Surfaces B: Biointerfaces 19: 291-295

Ziegler J.F., J.P. Biersack, U. Littmark. 1985. The Stopping and Range of Ions in Solids. Pergamon Press, Oxford 